\title{
Heat transfer and large scale dynamics in turbulent Rayleigh-Bénard convection
}

\author{
Guenter Ahlers* \\ Department of Physics and $1 Q C D$, University of California, Santa Barbara, California \\ 93106, USA \\ Siegfried Grossmann ${ }^{\dagger}$ \\ Fachbereich Physik, Philipps-Universität Marburg, D-35032 Marburg, Germany \\ Detlef Lohse $^{\ddagger}$ \\ Physics of Fluids Group, Department of Science and Technology, J. M. Burgers Centre \\ for Fluid Dynamics, and Impact-Institute, University of Twente, 7500 AE Enschede, \\ The Netherlands
}

(Published 22 April 2009)

\begin{abstract}
The progress in our understanding of several aspects of turbulent Rayleigh-Bénard convection is reviewed. The focus is on the question of how the Nusselt number and the Reynolds number depend on the Rayleigh number Ra and the Prandtl number Pr, and on how the thicknesses of the thermal and the kinetic boundary layers scale with $\mathrm{Ra}$ and Pr. Non-Oberbeck-Boussinesq effects and the dynamics of the large scale convection roll are addressed as well. The review ends with a list of challenges for future research on the turbulent Rayleigh-Bénard system.
\end{abstract}

DOI: $10.1103 /$ RevModPhys.81.503

PACS number(s): 47.27.te, 47.55.P-, 47.27.T-

\section{CONTENTS}

I. Introduction

II. Theories of Global Properties: The Nusselt and Reynolds Number

A. Older theories for $\mathrm{Nu}(\mathrm{Ra}, \mathrm{Pr})$ and $\mathrm{Re}(\mathrm{Ra}, \mathrm{Pr})$

B. Grossmann-Lohse theory for $\mathrm{Nu}(\mathrm{Ra}, \mathrm{Pr})$ and $\operatorname{Re}(\operatorname{Ra}, \operatorname{Pr})$

C. Is there an asymptotic regime for large Ra?, and strict upper bounds

III. Experimental Measurements of the Nusselt Number

A. Overview

B. Sidewall and top- and bottom-plate-conductivity effects on $\mathrm{Nu}$

C. The Nusselt number for $\operatorname{Pr} \simeq 4.38$ obtained using water as the fluid

D. The Prandtl-number dependence of the Nusselt number

E. The aspect-ratio dependence of the Nusselt number

F. The insensitivity of the Nusselt number to the LSC

G. The dependence of $\mathrm{Nu}$ on $\mathrm{Ra}$ at very large $\mathrm{Ra}$

IV. Experimental Measurements of the Reynolds Numbers

A. Reynolds numbers based on the large scale convection roll

B. Reynolds numbers based on plume motion

V. $\mathrm{Nu}(\mathrm{Ra}, \mathrm{Pr})$ and $\mathrm{Re}(\mathrm{Ra}, \mathrm{Pr})$ in Direct Numerical

*guenter@physics.ucsb.edu grossmann@physik.uni-marburg.de

†.lohse@utwente.nl
A. Relevance of boundary layers and challenges

Thermal boundary layers

C. Kinetic boundary layers

VII. Non-Oberbeck-Boussinesq Effects

VIII. Global Wind Dynamics

IX. Issues for Future Research $\quad 530$

\section{INTRODUCTION}

Rayleigh-Bénard (RB) convection-the buoyancy driven flow of a fluid heated from below and cooled from above-is a classical problem in fluid dynamics. It played a crucial role in the development of stability theory in hydrodynamics (Chandrasekhar, 1981; Drazin and Reid, 1981) and had been paradigmatic in pattern formation and in the study of spatial-temporal chaos (Getling, 1998; Bodenschatz et al., 2000). From an applied viewpoint, thermally driven flows are of utmost importance. Examples are thermal convection in the atmosphere [see, e.g., Hartmann et al. (2001)], in the oceans [see, e.g., Marshall and Schott (1999)] [including thermohaline convection; see, e.g., Rahmstorf (2000)], in buildings [see, e.g., Hunt and Linden (1999)], in process technology, and in metal-production processes [see, e.g., Brent et al. (1988)]. In the geophysical and astrophysical context, we mention convection in the Earth's mantle [see, e.g., McKenzie et al. (1974)], in the Earth's outer 
core [see, e.g., Cardin and Olson (1994)], and in stars including our Sun [see, e.g., Cattaneo et al. (2003)]. Convection has been associated with the generation and reversal of the Earth's magnetic field [see, e.g., Glatzmaier and Roberts (1995)].

Even if one restricts oneself to thermally driven flows in a closed box, there are so many aspects that not all of them can be addressed in this single review. We focus on developed turbulence when spatial coherence throughout the cell is lost and only on the large scale dynamics of the flow and aspects intimately connected with it, such as the boundary layer structures. The scaling of the spectra of velocity and temperature fluctuations, or of the corresponding structure functions, will not be addressed. These issues had been discussed in the review by Siggia (1994), but meanwhile considerable progress has been achieved, in particular on the question of whether and where in the flow to expect BolgianoObukhov scaling (Bolgiano, 1959; Obukhov, 1959; Monin and Yaglom, 1975) of the structure functions; see, e.g., Calzavarini et al. (2002); Sun et al. (2006); Kunnen et al. (2008). For a very recent review on these issues, we refer to Lohse and Xia (2010).

The question to be asked about the Rayleigh-Bénard problem is as follows: For a given fluid in a closed container of height $L$ heated from below and cooled from above, what are the flow properties inside the container and, in addition, what is the heat transfer from bottom to top? Here spatially and temporally constant temperatures are assumed at the bottom and top. In Sec. III we discuss to what degree this assumption can be justified in reality (Chaumat et al., 2002; Verzicco, 2004; Brown, Funfschilling, et al., 2005).

The problem is further simplified by the so-called Oberbeck-Boussinesq (OB) approximation (Oberbeck, 1879; Boussinesq, 1903; Landau and Lifshitz, 1987) in which the fluid density $\rho$ is assumed to depend linearly on the temperature,

$$
\rho(T)=\rho\left(T_{0}\right)\left[1-\beta\left(T-T_{0}\right)\right],
$$

with $\beta$ the thermal expansion coefficient. In addition, it is assumed that the material properties of the fluid such as $\beta$, the viscosity $\nu$, and the thermal diffusivity $\kappa$ do not depend on temperature. The governing equations of the RB problem are then the Oberbeck-Boussinesq equations (Landau and Lifshitz, 1987)

$$
\begin{aligned}
& \partial_{t} u_{i}+u_{j} \partial_{j} u_{i}=-\partial_{i} p+\nu \partial_{j}^{2} u_{i}+\beta g \delta_{i 3} \theta, \\
& \partial_{t} \theta+u_{j} \partial_{j} \theta=\kappa \partial_{j}^{2} \theta
\end{aligned}
$$

for the velocity field $\boldsymbol{u}(\boldsymbol{x}, t)$, the kinematic pressure field $p(\boldsymbol{x}, t)$, and the temperature field $\theta(\boldsymbol{x}, t)$ relative to some reference temperature. Here and in the following we assume summation over double indices; $\delta_{i j}$ is the Kronecker symbol. The Oberbeck-Boussinesq equations are assisted by continuity $\partial_{i} u_{i}=0$ and the boundary conditions $\boldsymbol{u}=\mathbf{0}$ for the velocities at all walls, $\theta(z$ $=-L / 2)=\Delta / 2$ for the temperature at the bottom plate, and $\theta(z=L / 2)=-\Delta / 2$ for the temperature at the top plate. At the sidewalls the condition of no lateral heat flow is imposed. The limitations of the OberbeckBoussinesq approximations are discussed in Sec. VII.

Within the OB approximation and for a given cell geometry, the system is determined by only two dimensionless control parameters, namely, the Rayleigh number and the Prandtl number,

$$
\mathrm{Ra}=\frac{\beta g L^{3} \Delta}{\kappa \nu}, \quad \operatorname{Pr}=\frac{\nu}{\kappa} .
$$

The cell geometry is described by its symmetry and one or more aspect ratios $\Gamma$. For a cylindrical cell $\Gamma \equiv d / L$, where $d$ is the cell diameter.

The key response of the system to the imposed $\mathrm{Ra}$ is the heat flux $H$ from bottom to top. The dimensionless heat flux $\mathrm{Nu}=H / \Lambda \Delta L^{-1}$ is the Nusselt number. Here $\Lambda$ $=c_{p} \rho \kappa$ is the thermal conductivity. Within the OberbeckBoussinesq approximation one obtains for incompressible flow

$$
\mathrm{Nu}=\frac{\left\langle u_{z} \theta\right\rangle_{A}-\kappa \partial_{3}\langle\theta\rangle_{A}}{\kappa \Delta L^{-1}} .
$$

Here $\langle\cdot\rangle_{A}$ denotes the average over (any) horizontal plane and over time. Correspondingly, $\langle\cdot\rangle_{V}$ used below denotes the volume and time average.

Another key system response is the extent of turbulence, expressed in terms of a characteristic velocity amplitude $U$, nondimensionalized by $\nu L^{-1}$ to define a Reynolds number

$$
\operatorname{Re}=\frac{U}{\nu L^{-1}} .
$$

As we show in Sec. IV, there are various reasonable possibilities to choose a velocity, e.g., the components or the magnitude of the velocity field at different positions, local or averaged amplitudes, turnover times or frequency peaks in the thermal spectrum, etc. In some parameter ranges these amplitudes differ not only in magnitude but even show different dependences on $\mathrm{Ra}$ and $\operatorname{Pr}$ (Brown et al., 2007; Sugiyama et al., 2009). Mostly we restrict ourselves to that Reynolds number which is associated with the large scale circulation (LSC), also called the "wind of turbulence" $U$ (Niemela et al., 2001; Xia et al., 2003; Sun, Xia, and Tong, 2005). There is discussion in the literature whether or not the LSC evolves out of the well-known cellular structures at small Ra. On the one hand, Krishnamurti and Howard (1981) performed experiments from which they concluded that the LSC is not a simple reminder and continuation of the roll structure observed just after the onset of convection. On the other hand, we are not aware that their observations have been confirmed. Even an explicit search for such a mode as Krishnamurti and Howard (1981) reported was not successful; see the review by Busse (2003) and Hartlep et al. (2005). They concluded that the LSC at large $\mathrm{Ra}$ indeed is a reminder of the low Ra structures. The dynamics of the large scale wind, its azimuthal oscillation, diffusion, reorientation, cessation, and possible 


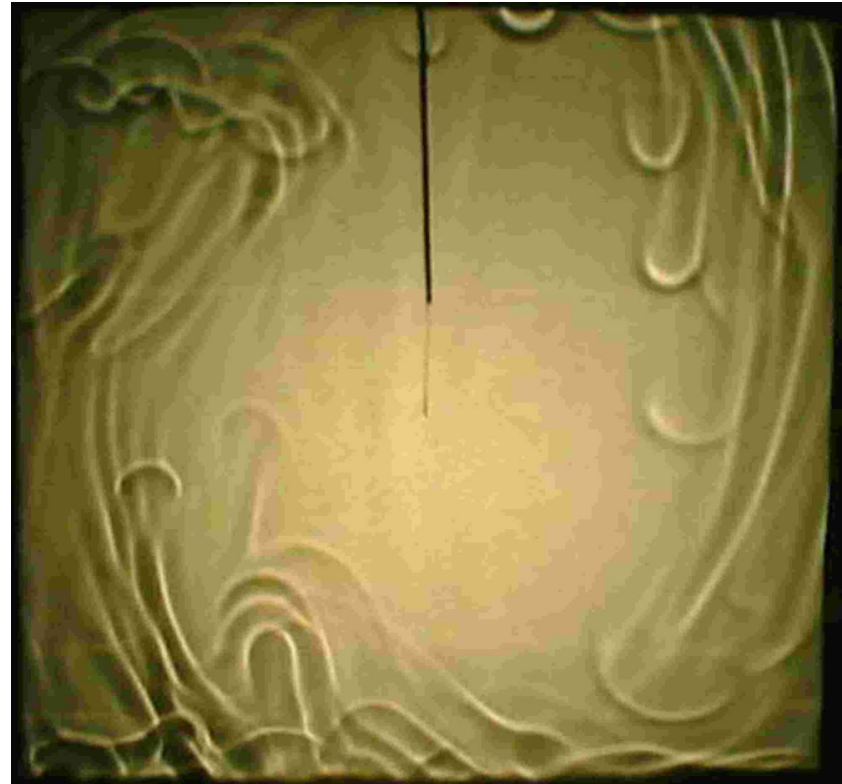

(a)

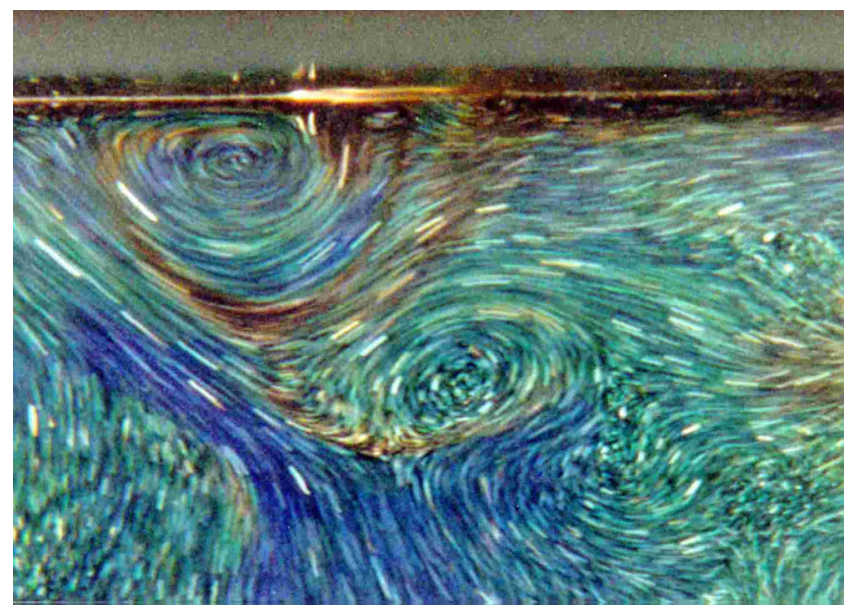

(b)

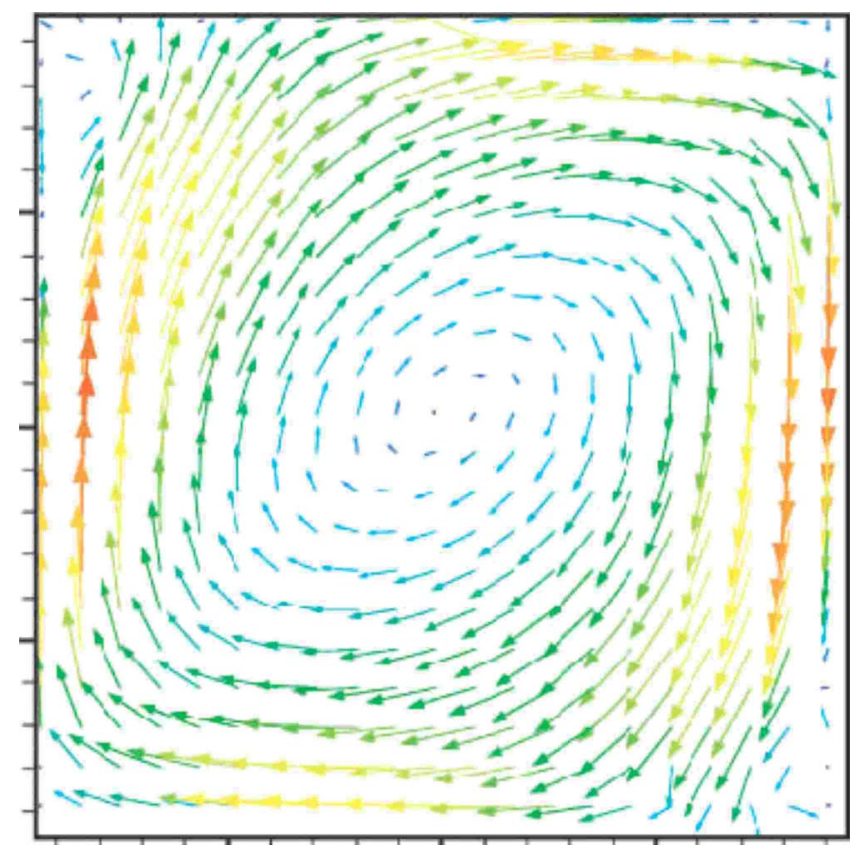

(c)

FIG. 1. (Color online) Plumes and flow field. (a) Shadowgraph visualization of rising and falling plumes at $\mathrm{Ra}=6.8 \times 10^{8}$, Pr $=596$ (dipropylene glycol) in a $\Gamma=1$ cell. From Shang et al., 2003. (b) Streak picture of temperature sensitive liquid crystal spheres taken near the top cold surface in a $\Gamma=1$ cell at $\mathrm{Ra}=2.6 \times 10^{9}$ and $\mathrm{Pr}=5.4$ (water), in order to visualize plume detachment. The view shows an area of $6.5 \mathrm{~cm} \times 4 \mathrm{~cm}$. From Du and Tong, 2000. (c) Time-averaged velocity-vector map in the plane of the LSC at $\mathrm{Ra}=7.0 \times 10^{9}$. Adapted from Sun, Xia, and Tong, 2005.

breakdown at very large $\mathrm{Ra}$ are discussed in detail in Sec. VIII.

The key question to ask is: How do $\mathrm{Nu}$ and $\mathrm{Re}$ depend on $\mathrm{Ra}$ and $\mathrm{Pr}$ ? The experimental situation will be the subject of Sec. III for $\mathrm{Nu}(\mathrm{Ra}, \mathrm{Pr})$ and $\mathrm{Sec}$. IV for $\mathrm{Re}(\mathrm{Ra}, \mathrm{Pr})$. Results from numerical simulations are reported in Sec. V. However, first (Sec. II) we summarize older theories (Sec. II.A) and then (Sec. II.B) the Grossmann-Lohse (GL) theory (Grossmann and Lohse, 2000, 2001, 2002, 2004). In Sec. II.C we discuss theories about a possible asymptotic regime at very large $\mathrm{Ra}$ and strict upper bounds for $\mathrm{Nu}$.

Section VI is devoted to the structure and width of the thermal and kinetic boundary layers (BLs). The thermal
BLs play a crucial role in determining the heat transfer, and the kinetic BLs provide viscous dissipation of the LSC. Another important feature is the thermal plumes (Zocchi et al., 1990; Kadanoff, 2001; Funfschilling and Ahlers, 2004; Xi et al., 2004; Zhou et al., 2007b) that detach from the thermal boundary layers; they contribute to the driving of the flow. In order to give an idea of the importance and organization of these plumes and their shapes (for large Pr) we show their shadowgraph visualization in Fig. 1(a), taken from Shang et al. (2003). Figure 1(b) shows a streak picture of the temperature distribution close to the upper plate, including a detaching plume for medium Pr. Figure 1(c) is a velocity-vector map of the LSC. 
TABLE I. Power-law exponents for $\mathrm{Nu}$ and $\mathrm{Re}$ as functions of $\mathrm{Ra}$ and Pr predicted by theories developed prior to the review by Siggia (1994). The exponents are defined by Eqs. (7) and (8). Whereas $\mathrm{Re}$ is based on the large scale wind velocity, $\mathrm{Re}_{\text {fluct }}$ is based on the velocity fluctuations.

\begin{tabular}{|c|c|c|c|c|c|c|}
\hline Reference & Pr and $\mathrm{Ra}$ range & $\gamma_{\mathrm{Nu}}$ & $\alpha_{\mathrm{Nu}}$ & $\gamma_{\mathrm{Re}}$ & $\gamma_{\mathrm{Re}_{\text {fluct }}}$ & $\alpha_{\mathrm{Re}}$ \\
\hline Davis (1922a, 1922b) & Ra small & $1 / 4$ & & & & \\
\hline Malkus (1954) & & $1 / 3$ & & & & \\
\hline \multirow[t]{2}{*}{ Kraichnan (1962) } & $\begin{array}{l}\text { Ra ultimate } \\
\quad \operatorname{Pr}<0.15\end{array}$ & $1 / 2$ & $1 / 2$ & $1 / 2$ & & $-1 / 2$ \\
\hline & $\begin{array}{l}\text { Ra ultimate, } \\
0.15<\operatorname{Pr} \lesssim 1\end{array}$ & $1 / 2$ & $-1 / 4$ & $1 / 2$ & & $-3 / 4$ \\
\hline Spiegel (1971) & Ra ultimate & $1 / 2$ & $1 / 2$ & $1 / 2$ & & $-1 / 2$ \\
\hline Castaing et al. (1989) & & $2 / 7$ & & $1 / 2$ & $3 / 7$ & \\
\hline Shraiman and Siggia (1990) & $\operatorname{Pr}>1$ & $2 / 7$ & $-1 / 7$ & $3 / 7$ & & $-5 / 7$ \\
\hline Yakhot (1992) & & $5 / 19$ & & & $8 / 19$ & \\
\hline Zaleski (1998) & & $2 / 7$ & & & & \\
\hline Cioni et al. (1997) & $\operatorname{Pr}<1$ & $2 / 7$ & $2 / 7$ & & $3 / 7$ & $-4 / 7$ \\
\hline
\end{tabular}

As mentioned, Sec. VII is devoted to non-OberbeckBoussinesq effects and Sec. VIII deals with the global wind dynamics. In Sec. IX we outline some major issues in Rayleigh-Bénard convection for future research.

\section{THEORIES OF GLOBAL PROPERTIES: THE NUSSELT AND REYNOLDS NUMBER}

\section{A. Older theories for $\mathrm{Nu}(\operatorname{Ra}, \operatorname{Pr})$ and $\operatorname{Re}(\operatorname{Ra}, \operatorname{Pr})$}

For a detailed discussion of the theories developed prior to the review by Siggia (1994) we refer the reader to that paper and to Chandrasekhar (1981). These theories predicted power laws

$$
\begin{aligned}
& \mathrm{Nu} \sim \operatorname{Ra}^{\gamma_{\mathrm{Nu}} \operatorname{Pr}^{\alpha_{\mathrm{Nu}}},} \\
& \mathrm{Re} \sim \mathrm{Ra}^{\gamma_{\mathrm{Re}} \operatorname{Pr}^{\alpha_{\mathrm{Re}}}}
\end{aligned}
$$

for the dependences of $\mathrm{Nu}$ and $\mathrm{Re}$ on $\mathrm{Ra}$ and Pr. A summary of predicted exponents is given in Table I. Early experiments were of limited precision, and were consistent with power-law dependences over their limited ranges of $\mathrm{Ra}$ and $\mathrm{Pr}$.

The conceptually easiest early theory is Malkus' marginal-stability theory of 1954 . It assumed that the thermal BL thickness adjusts itself so as to yield a critical BL Rayleigh number. This immediately gave $\gamma_{\mathrm{Nu}}$ $=1 / 3$. After the experiments by $\mathrm{Chu}$ and Goldstein (1973), Threlfall (1975) and the later ground-breaking Chicago experiments in cryogenic helium (Heslot et al., 1987; Castaing et al., 1989; Wu et al., 1990; Sano et al., 1989; Procaccia et al., 1991) had suggested a smaller power-law exponent, the Chicago group developed the mixing-zone model (Castaing et al., 1989) which later was extended by Cioni et al. (1997) to include the Prandtl-number dependences. The central result was $\gamma_{\mathrm{Nu}}=2 / 7$. The same scaling exponent could also be obtained from the BL theory of Shraiman and Siggia (1990), assuming a turbulent boundary layer. The as- sumptions of that theory are, however, very different from those of the mixing-layer theory, leading to very different power-law exponents for the dependences on the Prandtl number; see Table I.

As we show later, the assumption of a fully developed turbulent $\mathrm{BL}$ is far from being fulfilled in the parameter regime of the Chicago experiments. That can already be seen from an estimate of the coherence length $\ell$ of the RB flow. Taking the data from Procaccia et al. (1991) for the scaling of the velocity fluctuations and of the crossover frequency to the viscous subrange, Grossmann and Lohse (1993) obtained $\ell / L \approx 50 \mathrm{Ra}^{-0.32}$. For the $\Gamma=1 / 2$ cell of Procaccia et al. (1991) this implies that only at $\mathrm{Ra} \approx 10^{8}$ the coherence length becomes about $1 / 3$ of the lateral cell width and $1 / 6$ of its height, a pre-requisite for independent fluctuations to develop in the bulk. Estimates based on $\ell \approx 10 \eta$, where $\eta$ is the (locally or globally defined) Kolmogorov scale, give similar results. The transition to turbulence in the BL is correspondingly expected only at much large $\mathrm{Ra}$, namely, at $\mathrm{Ra}$ $\approx 10^{14}$ (at the edge of the achievable regime in the Chicago experiments), as we show in the next section.

In any case, at "large enough" Rayleigh number a transition should occur towards an ultimate Rayleighnumber regime. Such a regime was first suggested by Kraichnan (1962). Spiegel (1971) hypothesized that in that regime the heat flux and the turbulence intensity are independent of the kinematic viscosity and the thermal diffusivity, which leads to $\gamma_{\mathrm{Nu}}=1 / 2$ (for more details, see Sec. II.C). Though in those days (1971 and before) no measured power-law exponent was even close to that value, that paper has been extremely influential, perhaps also because from a mathematical point of view no lower strict upper bound than $\gamma_{\mathrm{Nu}}=1 / 2$ could be proven to exist for finite $\operatorname{Pr}$ [see Doering and Constantin (1996)].

As shown in Sec. III and IV, the experiments of the last decade reveal the limitations of most of these older theories. 


\section{B. Grossmann-Lohse theory for $\mathrm{Nu}(\operatorname{Ra}, \operatorname{Pr})$ and $\operatorname{Re}(\operatorname{Ra}, \operatorname{Pr})$}

Given the increasing richness and precision of experimental and numerical data for $\mathrm{Nu}(\mathrm{Ra}, \mathrm{Pr})(\mathrm{Sec}$. III) and $\operatorname{Re}(\mathrm{Ra}, \mathrm{Pr})(\mathrm{Sec} . \mathrm{IV})$, it became clear near the end of the last decade that none of the theories developed up to then could offer a unifying view, accounting for all data. In particular, the predicted Prandtl-number dependences of $\mathrm{Nu}$ (Shraiman and Siggia, 1990; Cioni et al., 1997) are in disagreement with measured and calculated data. Therefore in a series of papers, Grossmann and Lohse $(2000,2001,2002,2004)$ tried to develop a unifying theory to account for $\mathrm{Nu}(\mathrm{Ra}, \mathrm{Pr})$ and $\mathrm{Re}(\mathrm{Ra}, \mathrm{Pr})$ over wide parameter ranges.

The backbone of the theory is a set of two exact relations for the kinetic and thermal energy-dissipation rates $\epsilon_{u}$ and $\epsilon_{\theta}$, respectively, namely,

$$
\begin{aligned}
\epsilon_{u} & \equiv\left\langle\nu\left[\partial_{i} u_{j}(\boldsymbol{x}, t)\right]^{2}\right\rangle_{V}=\frac{\nu^{3}}{L^{4}}(\mathrm{Nu}-1) \mathrm{RaPr}^{-2}, \\
\epsilon_{\theta} & \equiv\left\langle\kappa\left[\partial_{i} \theta(\boldsymbol{x}, t)\right]^{2}\right\rangle_{V}=\kappa \frac{\Delta^{2}}{L^{2}} \mathrm{Nu} .
\end{aligned}
$$

These relations can easily be derived from the Boussinesq equations and the corresponding boundary conditions [see, e.g., Shraiman and Siggia (1990)], assuming only statistical stationarity. The central idea of the theory now is to split the volume averages of both the kinetic and the thermal dissipation rate into respective bulk and boundary layer (or rather boundary-layer-like) contributions,

$$
\begin{gathered}
\epsilon_{u}=\epsilon_{u, \mathrm{BL}}+\epsilon_{u, \text { bulk }}, \\
\epsilon_{\theta}=\epsilon_{\theta, \mathrm{BL}}+\epsilon_{\theta, \text { bulk }} .
\end{gathered}
$$

The motivation for this splitting is that the physics of the bulk and the BL (or BL-like) contributions to the dissipation rates is fundamentally different and thus the corresponding dissipation rate contributions must be modeled in different ways. The phrase "BL-like" indicates that from a scaling point of view we consider the detaching thermal plumes as parts of the thermal BLs. Thus instead of BL and bulk we could also use the labels pl (plume) and bg (background) for the two parts of the dissipation rates. A sketch of the splitting is shown in Fig. 2. Rather than Eq. (12) one therefore could also write

$$
\epsilon_{\theta}=\epsilon_{\theta, \mathrm{pl}}+\epsilon_{\theta, \mathrm{bg}},
$$

signaling the contributions from the $\mathrm{BL}$ and the plumes $(\mathrm{pl})$, on the one hand, and from the background (bg), on the other hand.

Two further assumptions of the GL theory are indicated as well in Fig. 2, namely, that there exists a large scale wind with only one typical velocity scale $U$ (defining a Reynolds number $\operatorname{Re}=U L / \nu$ ), and that the kinetic BLs are (scalingwise) characterized by a single effective thickness $\lambda_{u}$ regardless of the position along the plates and walls in the flow. As we show in Sec. IV for the
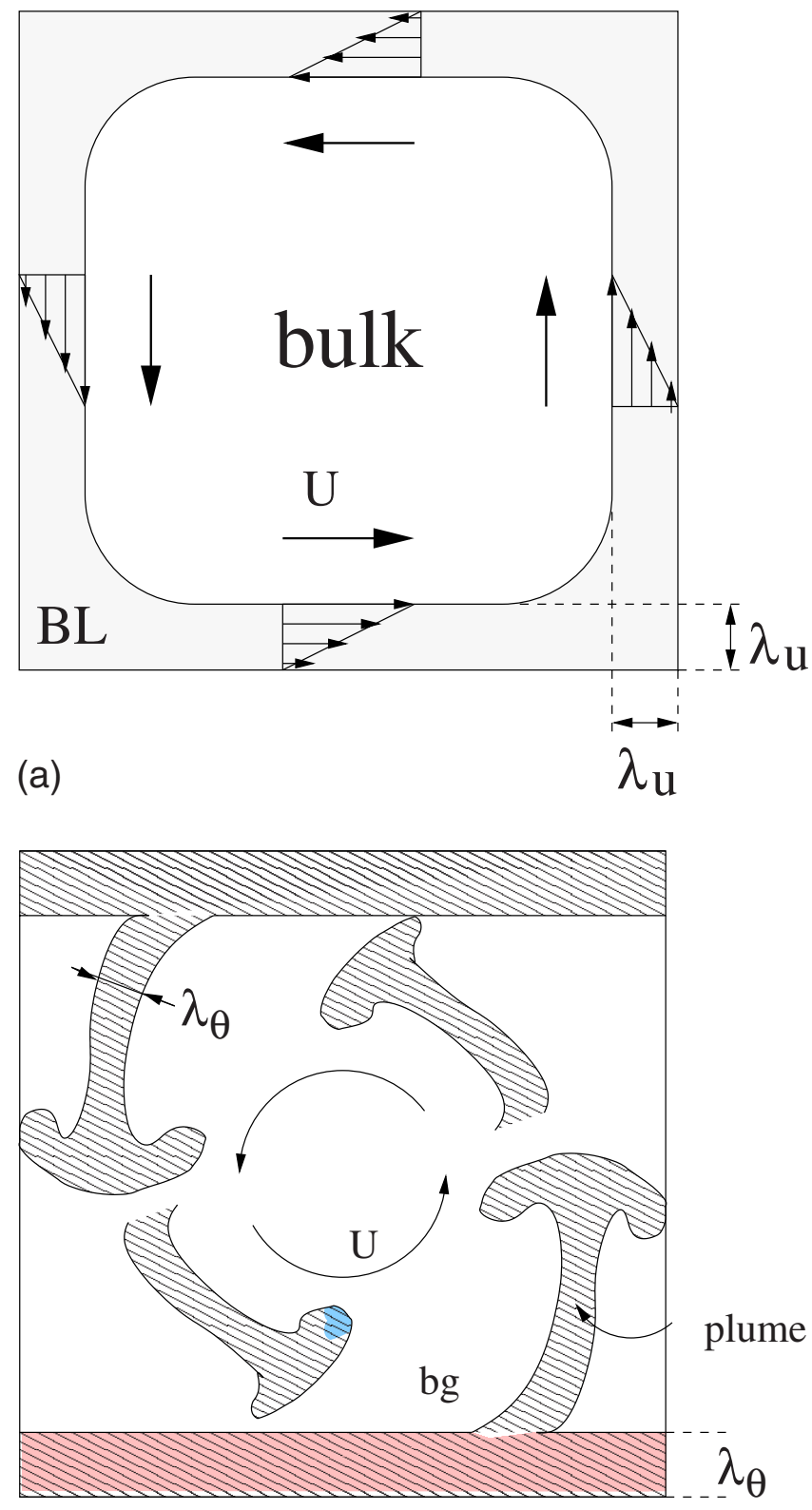

(b)

FIG. 2. (Color online) Boundary-bulk partition. Sketch of the splitting of the kinetic (a) and thermal (b) dissipation rates on which the GL theory is based. In both figures the large scale convection roll with typical velocity amplitude $U$ is sketched. The typical width of the kinetic BL is $\lambda_{u}$, whereas the typical thermal BL thicknesses and the plume thicknesses are $\lambda_{\theta}$. Outside the $\mathrm{BL} /$ plume region is the background flow (bg).

velocity scales and in Sec. VI for the BL thicknesses, both assumptions are simplifications. In particular, even the scaling of the kinetic BL thickness with Ra may be different at the sidewalls as compared to the top and bottom plates [see Xin et al. (1996), Xin and Xia (1997), Lui and Xia (1998), Qiu and Xia (1998b)]. Nevertheless, for the sake of simplicity and in view of Occam's razorand consistent with the recent experimental results for the BLs by Sun et al. (2008) - these simplifications have been used.

Accepting the splitting (11) and (12) [or Eq. (13)], the immediate consequence is that there are four main re- 
gimes in parameter space: regime I in which both $\epsilon_{u}$ and $\epsilon_{\theta}$ are dominated by the BL-plume contribution, regime II in which $\epsilon_{u}$ is dominated by $\epsilon_{u \text {,bulk }}$ and $\epsilon_{\theta}$ by $\epsilon_{\theta, \mathrm{BL}}$, regime III in which $\epsilon_{u}$ is dominated by $\epsilon_{u, \mathrm{BL}}$ and $\epsilon_{\theta}$ by $\epsilon_{\theta \text {,bulk }}$, and finally regime IV in which both $\epsilon_{u}$ and $\epsilon_{\theta}$ are dominated by their bulk contributions. It remains to be determined where in Ra-Pr parameter space the crossovers between the different regimes are located.

The next step is to model the individual contributions to the dissipation rates. We start with the bulk contributions. The turbulence in the bulk is driven by the large scale wind $U$. The corresponding time scale therefore is $L / U$, and from Kolmogorov's energy-cascade picture [see, e.g., Frisch (1995)] the bulk energy dissipation rate scalingwise becomes ${ }^{1}$

$$
\epsilon_{u, \text { bulk }} \sim \frac{U^{3}}{L}=\frac{\nu^{3}}{L^{4}} \operatorname{Re}^{3} .
$$

This seems justified because the turbulence in the bulk is more or less homogeneous and isotropic (Sun et al., 2006; Zhou, Sun, et al., 2008). The same reasoning can be applied to the temperature equation; see Frisch (1995). The bulk thermal dissipation rate then becomes

$$
\epsilon_{\theta, \text { bulk }} \sim \frac{U \Delta^{2}}{L}=\kappa \frac{\Delta^{2}}{L^{2}} \operatorname{PrRe} .
$$

The scaling of the boundary-layer contributions to the dissipation rates are estimated from their definitions as BL averages $\epsilon_{u, \mathrm{BL}}=\nu\left\langle\left[\partial_{i} u_{j}(\boldsymbol{x} \in \mathrm{BL}, t)\right]^{2}\right\rangle_{V}$ and $\boldsymbol{\epsilon}_{\theta, \mathrm{BL}}$ $=\kappa\left\langle\left[\partial_{i} \theta(\boldsymbol{x} \in \mathrm{BL}, t)\right]^{2}\right\rangle_{V}$, namely,

$$
\epsilon_{u, \mathrm{BL}} \sim \nu \frac{U^{2}}{\lambda_{u}^{2}} \frac{\lambda_{u}}{L}
$$

and

$$
\epsilon_{\theta, \mathrm{BL}} \sim \kappa \frac{\Delta^{2}}{\lambda_{\theta}^{2}} \frac{\lambda_{\theta}}{L} .
$$

As detailed by Grossmann and Lohse (2004), the kinetic and thermal BL thicknesses $\lambda_{u}$ and $\lambda_{\theta}$ are obtained from the Prandtl-Blasius BL theory (Prandtl, 1905; Blasius, 1908; Meksyn, 1961; Schlichting and Gersten, 2000; Cowley, 2001):

$$
\frac{\lambda_{u}}{L}=a \mathrm{Re}^{-1 / 2},
$$

where $a$ is a dimensionless prefactor of order 1 , and

$$
\frac{\lambda_{\theta}}{L} \sim \begin{cases}\mathrm{Re}^{-1 / 2} \operatorname{Pr}^{-1 / 2} & \text { for } \operatorname{Pr} \ll 1, \\ \operatorname{Re}^{-1 / 2} \operatorname{Pr}^{-1 / 3} & \text { for } \operatorname{Pr} \gg 1\end{cases}
$$

Note that scalingwise laminar BL theory is applied which seems justified because of the low prevailing boundary Reynolds numbers. Further below it will be estimated when this assumption breaks down for increasing Re. In the small Pr regimes [Eq. (19)] (label $l$

\footnotetext{
${ }^{1}$ Note that the Bolgiano-Obukhov length scale does not enter here.
}
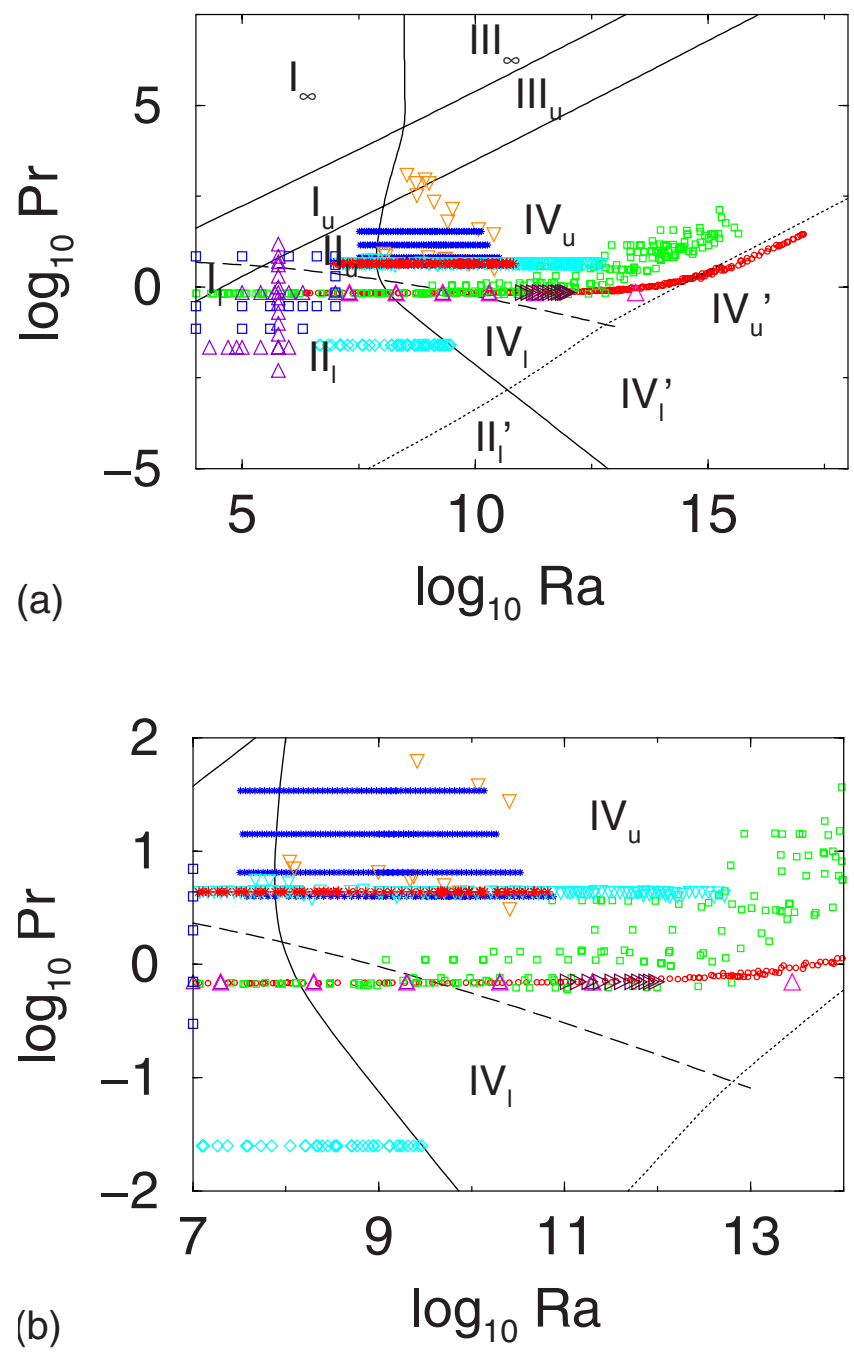

FIG. 3. (Color online) Phase diagram in Ra-Pr plane. (a) Phase diagram in the Ra-Pr plane according to Grossmann and Lohse (2000, 2001, 2002, 2004): The upper solid line means $\mathrm{Re}=\mathrm{Re}_{c}$; the lower nearly parallel solid line corresponds to $\epsilon_{u, \mathrm{BL}}=\epsilon_{u \text {, bulk }}$; the curved solid line is $\epsilon_{\theta, \mathrm{BL}}=\epsilon_{\theta, \text { bulk }}$; and along the long-dashed line $\lambda_{u}=\lambda_{\theta}$, i.e., $2 a \mathrm{Nu}=\sqrt{\mathrm{Re}}$. The dotted line indicates where the laminar kinetic BL is expected to become turbulent, based on a critical shear Reynolds number $\mathrm{Re}_{s}^{*}$ $\approx 420$ of the kinetic BL; cf. Landau and Lifshitz (1987). Data points where $\mathrm{Nu}$ has been measured or numerically calculated have been included (for aspect ratios $\Gamma \approx 1 / 2-1$ ): squares, Chavanne et al. (1997); diamonds, Cioni et al. (1997); circles, Niemela et al. (2000a); stars, Ahlers and Xu (2001); stars, Funfschilling et al. (2005), Nikolaenko et al. (2005); triangles down, Xia et al. (2002); triangles down, Sun, Xi, et al. (2005); triangles right, du Puits, Resagk, Tilgner, et al. (2007); triangles up, Verzicco and Camussi (1999) (numerical simulations); squares, Kerr and Herring (2000) (numerical simulations); triangles up, Amati et al. (2005), Verzicco and Sreenivasan (2008) (numerical simulations). Note that some of the large Ra data probably are influenced by NOB effects. (b) An enlargement of part (a).

stands for lower in Fig. 3) the kinetic BL is nested in the thermal one, $\lambda_{u}<\lambda_{\theta}$, whereas in the large Pr regimes [Eq. (20)] ( $u$ for upper in Fig. 3) the thermal BL is nested in the kinetic one, $\lambda_{\theta}<\lambda_{u}$. The transition from one re- 
gime to the other is modeled "by hand" through a crossover function $f\left(x_{\theta}\right)=\left(1+x_{\theta}^{4}\right)^{-1 / 4}$ of the variable $x_{\theta}=\lambda_{u} / \lambda_{\theta}$ $=2 a \mathrm{Nu} / \mathrm{Re}^{1 / 2}$; see Grossmann and Lohse (2001). Note that in the crossover function $f\left(\lambda_{u} / \lambda_{\theta}\right)$ the thermal BL thickness $\lambda_{\theta}$ has been replaced by $L /(2 \mathrm{Nu})$. Finally, when Re becomes very small the expression (18) for the kinetic BL thickness diverges, while the physical $\lambda_{u}$ is limited instead by an outer length scale of the order of the cell height $L$. This saturation is happening at some small but a priori unknown Reynolds number $\operatorname{Re}_{c}$. The transition towards the saturation regime is again modeled by hand with the crossover function $g\left(x_{L}\right)$ $=x_{L}\left(1+x_{L}^{4}\right)^{-1 / 4}$ with $x_{L}=\lambda_{u}(\operatorname{Re}) / \lambda_{u}\left(\operatorname{Re}_{c}\right)=\sqrt{\operatorname{Re}_{c} / \operatorname{Re}}$ [see Grossmann and Lohse (2001) for details].

When putting the splitting and modeling assumptions together with the two exact relations (9) and (10), one finally obtains two implicit equations for $\mathrm{Nu}(\mathrm{Ra}, \mathrm{Pr})$ and $\operatorname{Re}(\mathrm{Ra}, \operatorname{Pr})$ with six free parameters $a, \mathrm{Re}_{c}$, and $c_{i}, i$ $=1,2,3,4$ :

$$
\begin{aligned}
(\mathrm{Nu}-1) & \operatorname{RaPr}^{-2}=c_{1} \frac{\mathrm{Re}^{2}}{g\left(\sqrt{\mathrm{Re}_{c} / \mathrm{Re}}\right)}+c_{2} \operatorname{Re}^{3}, \\
\mathrm{Nu}-1= & c_{3} \operatorname{Re}^{1 / 2} \operatorname{Pr}^{1 / 2}\left\{f\left[\frac{2 a \mathrm{Nu}}{\sqrt{\mathrm{Re}_{c}}} g\left(\sqrt{\frac{\mathrm{Re}_{c}}{\mathrm{Re}_{\mathrm{e}}}}\right)\right]\right\}^{1 / 2} \\
& +c_{4} \operatorname{Pr} \operatorname{Re} f\left[\frac{2 a \mathrm{Nu}}{\sqrt{\mathrm{Re}_{c}}} g\left(\sqrt{\frac{\mathrm{Re}_{c}}{\mathrm{Re}}}\right)\right] .
\end{aligned}
$$

The -1 on the left-hand side of Eq. (22) stems from the contribution of the molecular transport, which survives when the Peclet number $\mathrm{Pe} \equiv \operatorname{RePr}=U L / \kappa$ tends to zero, $\mathrm{Pe} \rightarrow 0$; cf. Grossmann and Lohse (2008). This happens if either the velocity field decreases, $u_{i} \rightarrow 0$, or if the thermal diffusivity becomes large, $\kappa \rightarrow \infty$. In either case the time-averaged Oberbeck-Boussinesq equation (3) takes the form $\partial_{j}^{2} \theta=0$, whose solution with the proper boundary conditions is $\theta=-\Delta L^{-1} z$. Inserting this solution into the Nusselt number definition (5) gives $\lim _{P e \rightarrow 0} \mathrm{Nu}=1$. Of course the -1 does not matter much in the turbulent regime with large $\mathrm{Nu}$.

The six parameters in Eqs. (21) and (22) were adjusted so as to provide a fit to 155 data points for $\mathrm{Nu}(\mathrm{Ra}, \mathrm{Pr})$ from Ahlers and $\mathrm{Xu}$ (2001). These data were in the range $3 \times 10^{7} \leqslant \operatorname{Ra} \leqslant 3 \times 10^{9}$ and $4 \leqslant \operatorname{Pr} \leqslant 34$ for a $\Gamma=1$ cylindrical cell. As elaborated by Grossmann and Lohse (2002), in order to fix the parameter $a$ one also needs to know Re for (at least) one pair (Ra,Pr); Grossmann and Lohse (2002) took that value from Qiu and Tong (2001b). The final results were $a=0.482, c_{1}=8.7, c_{2}$ $=1.45, c_{3}=0.46, c_{4}=0.013$, and $\operatorname{Re}_{c}=1.0$. With this set the data of Ahlers and Xu (2001) were described very well. Later these data were adjusted for sidewall and plate corrections. However, the agreement with them as well as with additional data (Funfschilling et al., 2005) for Ra up to $3 \times 10^{10}$ and $\operatorname{Pr}=4.38$ (see Fig. 4) is still good (see Sec. III.C). For the $\mathrm{Nu}(\mathrm{Ra}, \mathrm{Pr})$ and $\mathrm{Re}(\mathrm{Ra}, \mathrm{Pr})$ predicted with these parameters over wide ranges of $\mathrm{Ra}$ and $\mathrm{Pr}$ we refer the reader to the figures given by Grossmann and

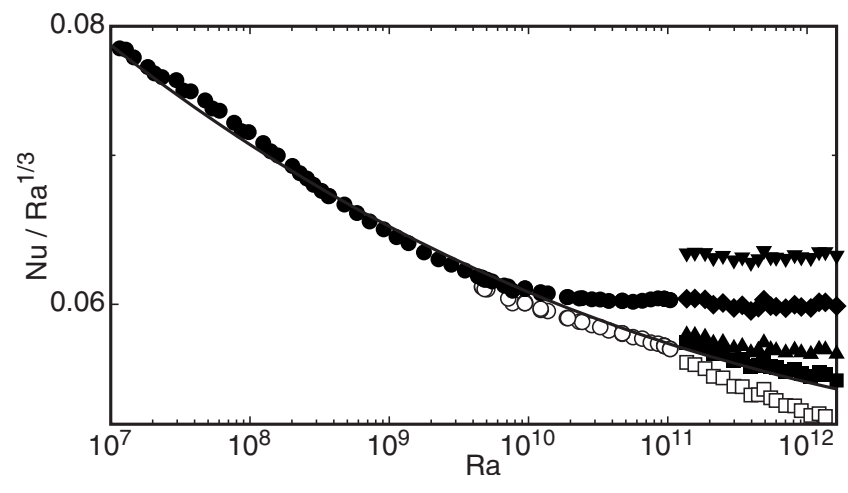

FIG. 4. Nusselt number versus Rayleigh number. Reduced $\mathrm{Nu}$ for $\Gamma=1$, obtained using water $(\operatorname{Pr} \approx 4.4)$ and copper plates, as a function of Ra. Open symbols, uncorrected data. Solid symbols, after correction for the finite plate conductivity. Circles, Funfschilling et al. (2005). Squares, Sun, Ren, et al. (2005). The downwards and upwards triangles are upper and lower bounds on the actual Nusselt number at large $\mathrm{Ra}$; the diamonds originate from an estimate, see the text for details. The solid line is the GL prediction (Grossmann and Lohse, 2001).

Lohse $(2001,2002)$. We mention that in principle one expects an aspect-ratio dependence of $c_{i}$, since the relative contributions of $\mathrm{BL}$ and bulk change with aspect ratio. However, from experiment it is known that the $\Gamma$ dependence of $\mathrm{Nu}(\mathrm{Ra}, \mathrm{Pr})$ is very weak in the explored range of $\mathrm{Ra}$ and $\mathrm{Pr}$ (see Sec. III.E).

After the determination of these six parameters, $\mathrm{Nu}(\mathrm{Ra}, \mathrm{Pr})$ and $\mathrm{Re}(\mathrm{Ra}, \mathrm{Pr})$ are given for all $\mathrm{Ra}$ and $\mathrm{Pr}$ by Eqs. (21) and (22). In addition, the Ra-Pr parameterspace structure with all transitions from one regime to another is also determined. The corresponding phase diagram is reproduced in Fig. 3; the respective "pure" power laws for $\mathrm{Nu}$ and $\mathrm{Re}$ of the various regimes are given in Table II.

One central assumption of the GL theory is the applicability of the scaling of the Prandtl-Blasius laminar BL theory. For increasing $\mathrm{Ra}$ and thus increasing $\mathrm{Re}$ this assumption will ultimately break down; the BLs are expected to become turbulent as well. Grossmann and Lohse $(2000,2002)$ provided an estimate for the Rayleigh number at which the breakdown occurs, based on the shear Reynolds number $\operatorname{Re}_{s}=\lambda_{u} U / \nu=a \sqrt{\operatorname{Re}}$. For RB experiments using classical fluids over "typical" Ra and Pr ranges $\mathrm{Re}_{s}$ is not particularly large. This reflects the relatively low degree of turbulence in the interior, which also becomes evident from flow visualizations similar to those by Tilgner et al. (1993), Xia et al. (2003), Funfschilling and Ahlers (2004), and Xi et al. (2004). For example, with $\operatorname{Pr} \simeq 4$ one has $\operatorname{Re}_{s}=15$ when $\mathrm{Ra}=10^{8}$ and $\mathrm{Re}$ $\approx 900$, and $\mathrm{Re}_{s}=190$ for $\mathrm{Ra}=10^{14}$ and $\mathrm{Re} \approx 140000$. The dotted line in Fig. 3 is based on the critical value $\mathrm{Re}_{s}^{*}$ $\simeq 420$. Beyond $\mathrm{Re}_{s}^{*}$ the kinetic BLs become fully turbulent and the Prandtl-Blasius scaling is no longer applicable. It is not totally clear what will happen in this ultimate regime of thermal convection. That will be discussed in the next section. 
TABLE II. The pure power laws for $\mathrm{Nu}$ and $\mathrm{Re}$ in the various regimes. From Grossmann and Lohse, 2001.

\begin{tabular}{ccccc}
\hline \hline Regime & Dominance of & $\mathrm{BLs}$ & $\mathrm{Nu}$ & $\mathrm{Re}$ \\
\hline $\mathrm{I}_{l}$ & $\epsilon_{u, \mathrm{BL}}, \epsilon_{\theta, \mathrm{BL}}$ & $\lambda_{u}<\lambda_{\theta}$ & $\mathrm{Ra}^{1 / 4} \operatorname{Pr}^{1 / 8}$ & $\mathrm{Ra}^{1 / 2} \operatorname{Pr}^{-3 / 4}$ \\
$\mathrm{I}_{u}$ & & $\lambda_{u}>\lambda_{\theta}$ & $\mathrm{Ra}^{1 / 4} \mathrm{Pr}^{-1 / 12}$ & $\mathrm{Ra}^{1 / 2} \operatorname{Pr}^{-5 / 6}$ \\
$\mathrm{I}_{\infty}$ & $\lambda_{u}=L / 4>\lambda_{\theta}$ & $\mathrm{Ra}^{1 / 5}$ & $\mathrm{Ra}^{3 / 5} \operatorname{Pr}^{-1}$ \\
$\mathrm{II}_{l}$ & $\epsilon_{u, \text { bulk }}, \epsilon_{\theta, \mathrm{BL}}$ & $\lambda_{u}<\lambda_{\theta}$ & $\mathrm{Ra}^{1 / 5} \mathrm{Pr}^{1 / 5}$ & $\mathrm{Ra}^{2 / 5} \operatorname{Pr}^{-3 / 5}$ \\
$\mathrm{II}_{u}$ & & $\lambda_{u}>\lambda_{\theta}$ & $\mathrm{Ra}^{1 / 5}$ & $\mathrm{Ra}^{2 / 5} \operatorname{Pr}^{-2 / 3}$ \\
$\mathrm{III}_{u}$ & $\epsilon_{u, \mathrm{BL}}, \epsilon_{\theta, \text { bulk }}$ & $\lambda_{u}>\lambda_{\theta}$ & $\mathrm{Ra}^{3 / 7} \operatorname{Pr}^{-1 / 7}$ & $\mathrm{Ra}^{4 / 7} \operatorname{Pr}^{-6 / 7}$ \\
$\mathrm{III}_{\infty}$ & & $\lambda_{u}=L / 4>\lambda_{\theta}$ & $\mathrm{Ra}^{1 / 3}$ & $\mathrm{Ra}^{2 / 3} \operatorname{Pr}^{-1}$ \\
$\mathrm{IV}_{l}$ & $\epsilon_{u, \text { bulk }}, \epsilon_{\theta, \text { bulk }}$ & $\lambda_{u}<\lambda_{\theta}$ & $\mathrm{Ra}^{1 / 2} \operatorname{Pr}^{1 / 2}$ & $\mathrm{Ra}^{1 / 2} \operatorname{Pr}^{-1 / 2}$ \\
$\mathrm{IV}_{u}$ & & $\lambda_{u}>\lambda_{\theta}$ & $\mathrm{Ra}^{1 / 3}$ & $\operatorname{Ra}^{4 / 9} \operatorname{Pr}^{-2 / 3}$ \\
\hline \hline
\end{tabular}

Note that the notion of laminar kinetic BLs in RB flow should not be confused with time independence or lack of chaotic behavior. The detaching thermal plumes introduce time dependences and chaotic behavior into the kinetic BL; but, as shown by Grossmann and Lohse (2004), the Prandtl-Blasius scaling laws for the thicknesses of the BLs still hold. Assuming a turbulent BL already for $\mathrm{Ra} \ll 10^{14}$ as done by Shraiman and Siggia (1990) leads to a dependence of $\mathrm{Nu}$ on Pr that disagrees with experiments and numerical simulations.

A detailed comparison of the GL theory with various data is given in Secs. III and IV. Here we stress only that the theory has predictive power: The determination of the free parameters was done in the limited parameter range $3 \times 10^{7} \leqslant \mathrm{Ra} \leqslant 3 \times 10^{9}$ and $4 \leqslant \operatorname{Pr} \leqslant 34$; see stars in Fig. 3. The predictions of the theory, however, hold over a much larger domain in the Ra-Pr parameter space.

We further note that due to Eq. (9) knowledge of the Nusselt number allows for an estimate of the volume averaged energy dissipation rate and derived quantities. For example, when taking the conditions of the Oregon cryogenic helium experiment (Niemela et al., 2000), for $\mathrm{Ra}=10^{10}$ and $\mathrm{Pr}=0.7$ one obtains either directly from experiment or from the GL theory a Nusselt number of 120 and with the experimental values $L=1 \mathrm{~m}$ and $\nu=5$ $\times 10^{-6} \mathrm{~m}^{2} / \mathrm{s}$ an energy dissipation rate of $\epsilon_{u}=3$ $\times 10^{-4} \mathrm{~m}^{2} / \mathrm{s}^{3}$. At $\mathrm{Ra}=10^{14}$ and $\operatorname{Pr}=0.7$ one obtains $\mathrm{Nu}$ $\approx 2400$ and with $\nu=10^{-7} \mathrm{~m}^{2} / \mathrm{s}$ a value of $\epsilon_{u}=5$ $\times 10^{-4} \mathrm{~m}^{2} / \mathrm{s}^{3}$. Both of these energy dissipation rates are about three orders of magnitude smaller than in typical wind tunnel experiments. From the volume-averaged energy dissipation rate equation (9) one can also obtain global estimates for the spatial coherence length $\ell$ which typically is about ten times the Kolmogorov length scale $\eta=\nu^{3 / 4} / \epsilon_{u}^{1 / 4}$. For example, for cryogenic helium ( $\operatorname{Pr}$ $=0.7)$ at $\mathrm{Ra}=10^{7}$ one obtains $\ell / L \approx 10 \eta / L$ $=10 \operatorname{Pr}^{1 / 2} /\left[(\mathrm{Nu}-1)^{1 / 4} \mathrm{Ra}^{1 / 4}\right] \approx 0.08$, which is small enough to allow for the loss of spatial coherence and the onset of turbulence in the bulk. In contrast, for the same Ra in water (at $\operatorname{Pr}=4$ ) one has $\ell / L \approx 0.18$ and in glycerol (at $\operatorname{Pr}=2000$ ) even at $\operatorname{Ra}=10^{9}$ one only has $\ell / L \approx 0.9$, so that there is no developed turbulence. In glycerol, only at
$\mathrm{Ra}=10^{11}$ one obtains $\ell / L \leqq 0.2$ and thus developed turbulence, according to this GL-model based estimate.

Finally, we note that the GL approach also has been applied to other geometries and flows: For example, Eckhardt et al. (2000, 2007a, 2007b) applied it to TaylorCouette and pipe flow and Tsai et al. $(2003,2005,2007)$ to turbulent electroconvection.

\section{Is there an asymptotic regime for large Ra?, and strict upper bounds}

Kraichnan (1962) later Spiegel (1971) postulated an "ultimate," or asymptotic, regime in which heat transfer and the strength of turbulence become independent of the kinematic viscosity and the thermal diffusivity. The physics of this ultimate regime is that the thermal and kinetic boundary layers, and thus the kinematic viscosity $\nu$ and the thermal diffusivity $\kappa$, do not play an explicit role any more for the heat flux. The flow then is bulk dominated. With proper nondimensionalization, and including logarithmic corrections due to viscous sublayers induced by no-slip boundary conditions, Kraichnan's predictions for this regime read

$$
\begin{aligned}
& \mathrm{Nu} \sim \mathrm{Ra}^{1 / 2}(\ln \mathrm{Ra})^{-3 / 2} \operatorname{Pr}^{1 / 2}, \\
& \mathrm{Re} \sim \mathrm{Ra}^{1 / 2}(\ln \mathrm{Ra})^{-1 / 2} \operatorname{Pr}^{-1 / 2},
\end{aligned}
$$

for $\operatorname{Pr}<0.15$, while for $0.15<\operatorname{Pr} \leqslant 1$ he suggested

$$
\begin{aligned}
& \mathrm{Nu} \sim \mathrm{Ra}^{1 / 2}(\ln \mathrm{Ra})^{-3 / 2} \mathrm{Pr}^{-1 / 4}, \\
& \mathrm{Re} \sim \mathrm{Ra}^{1 / 2}(\ln \mathrm{Ra})^{-1 / 2} \mathrm{Pr}^{-3 / 4} .
\end{aligned}
$$

The Ra-number dependences agree with the dependences in regimes $\mathrm{VI}_{l}$ and $\mathrm{VI}_{l}^{\prime}$ of the GL theory (Grossmann and Lohse, 2000, 2001, 2002, 2004), except for the logarithmic corrections. The Pr dependence within the GL theory in the ultimate regimes $\mathrm{VI}_{l}$ and $\mathrm{VI}_{l}^{\prime}$ is different:

$$
\mathrm{Nu} \sim \mathrm{Ra}^{1 / 2} \operatorname{Pr}^{1 / 2},
$$




$$
\operatorname{Re} \sim \operatorname{Ra}^{1 / 2} \operatorname{Pr}^{-1 / 2} .
$$

Equation (27) was derived first by Spiegel (1971) from a model for thermal convection in stars.

To illustrate the physical implications of the existence of the ultimate regime, Acrivos (2008) suggested the following gedanken experiment: Consider RB convection in a very large aspect ratio sample, with the lateral dimension (say, the diameter $D$ of a cylinder) much larger than the sample height $L$. Now fix all dimensional parameters $(\Delta, \kappa, \nu, \beta, g$, and $D)$ and increase the sample height $L$, starting from zero, but such that always still $D \gg L$, i.e., remain in the large aspect ratio limit. How does the dimensional heat flux $H=\mathrm{Nu} \Lambda \Delta / L$ behave? First, $H \sim L^{-1}$, corresponding to $\mathrm{Nu}=1$. With increasing $L$, the decrease will become weaker. The regime $\mathrm{Nu}$ $\sim \mathrm{Ra}^{1 / 3}$ corresponds to the dimensional heat flux $H$ being independent of $L$. For even further increase of $L$ (with still $D \gg L$ ), the existence of the ultimate regime $\mathrm{Nu} \sim \mathrm{Ra}^{1 / 2}$ would imply that the dimensional heat flux $H$ would increase again, namely, with $L^{1 / 2}$. This feature may be considered as counterintuitive. However, our interpretation of the ultimate regime (if it exists) is that, with fully developed turbulence in the bulk, the increasing sample height $L$ allows for larger and larger eddies which thus can transport more and more heat from the bottom to the top plate.

Ever since Kraichnan's prediction in 1962, researchers have tried to find evidence for this regime. Various experimental efforts are discussed in Sec. III.G.

There are also numerical indications of the ultimate regime: In order to obtain a $\mathrm{Nu} \sim \mathrm{Ra}^{1 / 2}$ power law, the classical velocity and temperature boundary conditions of the RB problem have been modified: Lohse and Toschi (2003) and Calzavarini et al. (2005) performed numerical simulations for so-called "homogeneous" RB turbulence, in which the top- and bottom-temperature boundary conditions have been replaced by periodic ones, with an unstratified temperature gradient imposed. The idea was to eliminate the BLs in this way. The numerical results of Calzavarini et al. (2005) -including the found Prandtl number dependence-are consistent with the ultimate scaling equations (27) and (28), where the Reynolds number is that of the velocity fluctuations. As pointed out by Calzavarini et al. (2006) however, one should note that the dynamical equations of homogeneous RB turbulence allow for exponentially growing (in time) solutions, i.e., homogeneous RB turbulence does not have any strict upper bound for $\mathrm{Nu}$.

Such upper bounds do exist for the classical RB problem. Building on Howard's seminal variational formulation (Howard, 1963, 1972), Busse (1969) could prove that $\mathrm{Nu} \leqslant(\mathrm{Ra} / 1035)^{1 / 2}$ for any Pr. Later Doering and Constantin (1996) derived a strict upper bound given by $\mathrm{Nu} \leqslant 0.167 \mathrm{Ra}^{1 / 2}-1$. They employed the so-called "background method" (Doering and Constantin, 1992). The hitherto absolute best asymptotic upper bound on $\mathrm{Nu}(\mathrm{Ra})$ comes from Plasting and Kerswell (2003), obtaining $\mathrm{Nu} \leqslant 1+0.02634 \mathrm{Ra}^{1 / 2}$, which is $20 \%$ lower than Busse's best estimate. For arbritary Pr no power-law ex- ponent of Ra smaller than $1 / 2$ could hitherto be obtained as an upper bound. However, for infinite Pr Constantin and Doering (1999) could prove that $\mathrm{Nu} \leqslant$ const $\times \mathrm{Ra}^{1 / 3}[\operatorname{ln~Ra}]^{2 / 3}$. This result was improved later to $\mathrm{Nu}$ $\leqslant 0.644 \times \mathrm{Ra}^{1 / 3}[\operatorname{ln~Ra}]^{1 / 3}$ by Doering et al. (2006). Otero et al. (2002) obtained a strict upper bound for $\mathrm{Nu}$ for $\mathrm{RB}$ convection with constant heat flux through the plates (rather than with constant temperatures of the plates), namely, $\mathrm{Nu} \leqslant$ const $\times \mathrm{Ra}^{1 / 2}$ also for this case. We note that the scaling laws resulting from the GL theory are compatible with the upper bounds, including those in the large-Pr limit.

\section{EXPERIMENTAL MEASUREMENTS OF THE NUSSELT NUMBER}

\section{A. Overview}

During the last two or three decades measurements of $\mathrm{Nu}(\mathrm{Ra})$ as a function of such parameters as $\Gamma$, the extent of departures from the OB approximation, the deliberate suppression of the large scale circulation (LSC) by internal obstructions, the roughness of the confining solid surfaces, or deliberate misalignment relative to gravity have revealed various aspects of the heattransport mechanisms involved in this system. These efforts received a significant boost when it was appreciated that liquid or gaseous helium at low temperatures offered experimental opportunities not available at ambient temperatures (Ahlers, 1974, 1975; Threlfall, 1975; Behringer, 1985; Niemela and Sreenivasan, 2006b). Extensive low-temperature measurements of $\mathrm{Nu}(\mathrm{Ra})$ were initiated by the Chicago group (Heslot et al., 1987; Castaing et al., 1989; Sano et al., 1989), followed by the Grenoble group (Chavanne et al., 1996, 1997, 2001; Roche, Castaing, Chabaud, and Hebral, 2001, 2002, 2004) and the Oregon-Trieste group (Niemela, Skrbek, Swanson, et al., 2000; Niemela et al., 2000a, 2000b, 2001; Niemela and Sreenivasan, 2003a, 2006a, 2006b). Among the advantages of the low-temperature environment is the exceptionally small shear viscosity of helium gas which, at sufficiently high density, permits the attainment of extremely large Ra. Further enhancements of the achievable $\mathrm{Ra}$ have be attained near the critical points of several fluids, including helium, where the thermal expansion coefficient diverges and the thermal diffusivity vanishes, yielding a diverging $\mathrm{Ra}$ at constant $\Delta$. Here, however, it must be noted that on average the increase of $\mathrm{Ra}$ is accompanied by an increase of $\operatorname{Pr}$ (see Fig. 6, bottom) because Pr diverges as well at the critical point. This makes it difficult to disentangle any influence of $\mathrm{Ra}$, on the one hand, and of $\mathrm{Pr}$, on the other hand, on this system. Another unique property of materials at low temperatures is the extremely small heat capacity and large thermal diffusivity of the confining top and bottom plates which permit the study of temperature fluctuations at the fluid-solid interface when the heat current is held constant and led to the observation of chaos in a system governed by continuum equations (Ahlers, 1974, 1975). Additional advances in recent times have been 
due to the application of precision measurements, using classical liquids and gases at ever increasing $\mathrm{Ra}$ ( $\mathrm{Xu}$ et al., 2000; Fleischer and Goldstein, 2002; Roche et al., 2002; Funfschilling et al., 2005; Nikolaenko et al., 2005; Sun, Ren, et al. 2005) and over a wide range of $\mathrm{Pr}$ (Ahlers and Xu, 2001; Xia et al., 2002).

\section{B. Sidewall and top- and bottom-plate-conductivity effects on $\mathrm{Nu}$}

A serious problem for quantitative measurements of $\mathrm{Nu}$ is the influence of the sidewall (Ahlers, 2000; Roche, Castaing, Chabaud, Hebral, and Sommeria, 2001; Verzicco, 2002; Niemela and Sreenivasan, 2003a). The wall is in thermal contact with the convecting fluid and shares with it, by virtue of the thermal BLs, a large vertical temperature gradient near the top and bottom and a much smaller gradient away from the plates. Thus the current entering and leaving the wall is larger for the filled sample than it is for the empty one. In the wall near the top and bottom ends there is also a lateral gradient that will cause a part of the wall current to enter the fluid in the bottom half of the sample, and to leave it again in the top half. This will influence the detailed nature of the LSC (Niemela and Sreenivasan, 2003a). However, the global heat current is determined primarily by processes within the top and bottom BLs. Thus it is insensitive to the detailed structure and intensity of the LSC and is not influenced much by this complicated lateral heat flow out of and into the wall. Therefore the problem reduces primarily to determining the current that actually enters the fluid. Approximate models that provide a correction for this wall effect have been proposed (Ahlers, 2000; Roche, Castaing, Chabaud, Hebral, and Sommeria, 2001), but these are of limited reliability when the effect is large. The cryogenic measurements have a disadvantage because the sample usually is contained by steel sidewalls that have a relatively large conductivity $\Lambda_{w} \simeq 0.2 \mathrm{~W} / \mathrm{m} \mathrm{K}$, while the fluid itself has an exceptionally small conductivity of order $0.01 \mathrm{~W} / \mathrm{m} \mathrm{K}$, giving $\Lambda_{w} / \Lambda \simeq 20$. In this case the models suggest that the correction is about $10 \%$ of $\mathrm{Nu}$ when $\mathrm{Nu} \simeq 100(\mathrm{Ra}$ $\simeq 4 \times 10^{9}$ ) and of course larger for smaller Nu. Even for $\mathrm{Ra} \simeq 10^{11}$ where $\mathrm{Nu} \simeq 280$ a correction of about $6 \%$ is suggested. The net result is that the measured effective exponent of $\mathrm{Nu}(\mathrm{Ra})$ is reduced below its true value by about 0.02 or 0.03 (Ahlers, 2000). For gases near ambient temperatures with typical thermal conductivities near $0.03 \mathrm{~W} / \mathrm{m} \mathrm{K}$, such as sulfur hexafluoride $\left(\mathrm{SF}_{6}\right)$ and ethane $\left(\mathrm{C}_{2} \mathrm{H}_{6}\right)$, confined by a high-strength-steel sidewall with a conductivity of $66 \mathrm{~W} / \mathrm{m} \mathrm{K}$ (Ahlers et al., 2007), one approaches the case of perfectly conducting lateral boundaries where subtraction of the current measured for the empty cell actually becomes a good approximation. Nonetheless, results for $\mathrm{Nu}$, although very precise, cannot be expected to be very accurate. An exceptionally favorable case is that of water confined by relatively thin plastic walls (Ahlers, 2000), where $\Lambda_{w} / \Lambda$ $\simeq 0.3$. In that case the sidewall correction can be as small as a fraction of a percent and may safely be ignored for most purposes. An intermediate case, for which reasonably reliable corrections can be made, is that of organic fluids confined by various plastic walls which typically have $\Lambda_{w} / \Lambda=\mathcal{O}(1)$ (Ahlers and $\mathrm{Xu}, 2001$ ). In the case of liquid metals, which are of interest because they have very small Prandtl numbers of order $10^{-2}$ or less, $\Lambda_{w} / \Lambda$ is small ( $\simeq 2$ for $\mathrm{Hg}$ and $\simeq 0.2$ for $\mathrm{Na}$ as examples) and again the wall corrections are small or negligible.

A second problem involves the finite conductivity $\Lambda_{p}$ of the top and bottom plates (Chaumat et al., 2002; Verzicco, 2004). One would like $X \equiv \Lambda_{p} L / e \Lambda$ Nu to be very large (here $e$ is the thickness of one plate). Else the emission of a plume from the top (bottom) boundary will leave an excess (deficiency) of enthalpy in its former location, generating a relatively warm (cold) spot near the plate where the probability of the emission of the next plume is diminished until this thermal "hole" has diffused away by virtue of the plate conductivity. This issue was explored experimentally by Brown, Funfschilling, et al. (2005) by measuring $\mathrm{Nu}(\mathrm{Ra})$ with high precision using water $(\Lambda \simeq 0.6 \mathrm{~W} / \mathrm{m} \mathrm{K})$ as the fluid and first $\mathrm{Al}$ and then $\mathrm{Cu}$ top and bottom plates of identical shape and size (see Fig. 4). The conductivities $\Lambda_{p, \mathrm{Cu}}$ $\simeq 400 \mathrm{~W} / \mathrm{m} \mathrm{K}$ of $\mathrm{Cu}$ and $\Lambda_{p, \mathrm{Al}} \simeq 170 \mathrm{~W} / \mathrm{m} \mathrm{K}$ of Al differ by a factor of about 2.3 and thus yield different reductions of $\mathrm{Nu}(\mathrm{Ra})$ below the ideal value $\mathrm{Nu}_{\infty}$ for isothermal boundary conditions. The results permitted the extrapolation of $\mathrm{Nu}$ to $\mathrm{Nu}_{\infty}$ by the use of the empirical formula

$$
\mathrm{Nu}=f(X) \mathrm{Nu}_{\infty}, \quad f(X)=1-\exp \left[-(a X)^{b}\right] .
$$

The parameters were $a=0.275$ and $b=0.39$ for $L$ $=0.50 \mathrm{~m}$, and $f(X)$ was closer to unity for smaller $L$. At fixed $L$ both $a$ and $b$ [and thus $f(X)$ ] were independent of $\Gamma$. This plate-conductivity effect is expected to be relatively small for the cryogenic and room-temperature compressed-gas experiments because typically $\Lambda_{p} / \Lambda$ $=\mathcal{O}\left(10^{4}\right)$ and larger and thus $X$ is very large unless $\mathrm{Nu}$ becomes extremely large. At modest $\mathrm{Ra}$, say $\mathrm{Ra} \leq 3$ $\times 10^{9}$, it is small also for $\mathrm{Cu}$ plates and organic fluids where $\Lambda_{p} / \Lambda=\mathcal{O}\left(10^{3}\right)$. The plate correction is a serious problem for measurements with liquid metals where for instance, $\Lambda_{p} / \Lambda \simeq 50$ for $\mathrm{Hg}$ and $\simeq 5$ for $\mathrm{Na}$. It has been suggested that this problem might be overcome using a composite plate containing a volume partially filled with a liquid of high vapor pressure. In that case the condensation and vaporization of this fluid inside the plate can yield an effective plate conductivity much larger than that of the metal alone. To our knowledge this idea has not yet been implemented.

The influence of the boundary conditions at the top and bottom plates was recently addressed through numerical simulations by Amati et al. (2005) and Verzicco and Sreenivasan (2008). Results for $\mathrm{Nu}(\mathrm{Ra})$ obtained with constant heat-flux boundary conditions (BCs) at the lower plate and constant-temperature BCs at the upper plate were compared with $\mathrm{Nu}(\mathrm{Ra})$ for constant- 
temperature BCs at both plates. The results for both BCs agreed reasonably well with each other and with experiment up to $\mathrm{Ra} \approx 10^{9}$. This is also found by comparing two-dimensional (2D) numerical simulations with constant temperature and constant flux BCs [Johnston and Doering $(2007,2009)]$. Beyond $\mathrm{Ra}=10^{9}$, early 3D numerical simulations (Amati et al., 2005; Verzicco and Sreenivasan, 2008) had suggested differences in the Nusselt numbers between constant-temperature and constant-flux BCs, with the former up to $30 \%$ larger than the latter and the experimental results. However, later numerical simulations with greater resolution revealed that the Nusselt numbers obtained from the numerical simulations with constant-temperature BCs are consistent with the constant-flux results and with the experimental data (Stevens, Verzicco, and Lohse, 2009). The conclusion is that constant-temperature and constant-flux boundary conditions within the present numerical accuracy lead to the same $\mathrm{Nu}$.

\section{The Nusselt number for $\operatorname{Pr} \simeq 4.38$ obtained using water as the fluid}

For $\operatorname{Pr} \simeq 4.4$ and $\Gamma=1.00$ high-accuracy measurements of $\mathrm{Nu}$ (Funfschilling et al., 2005) for $10^{7} \lesssim \mathrm{Ra} \leq 10^{11}$ using water and copper plates are shown in Fig. 4 as circles. We focus on these data because for them the sidewall corrections are negligible and top- and bottom-plate corrections based on experiments with plates of different conductivities were made (see Sec. III.B). The wide Ra range was achieved using three samples with different $L$. The data before the plate correction are given as open circles. Corrected data are presented as solid circles.

For $\Gamma=1$ and $\operatorname{Pr} \simeq 4$ the experiment reaching the largest $\mathrm{Ra}$ was conducted using $\mathrm{Cu}$ plates and a water sample with $L=100 \mathrm{~cm}$ and reached $\mathrm{Ra} \simeq 10^{12}$ (Sun, Ren, et al., 2005). These data are shown as open squares in the figure. It is gratifying that they are remarkably consistent with the open circles. However, they used an empirical plate correction with $a=0.987$ and $b=0.30$ which yielded the solid squares in the figure. In an attempt to develop an estimate of the uncertainty, we applied a correction using Eq. (29) and the parameters $a$ $=0.275$ and $b=0.39$ obtained from the $L=0.5 \mathrm{~m}$ sample. This yielded the up-pointing triangles. This correction is too small because measurements with a $L=0.25 \mathrm{~m}$ sample and the $L=0.50 \mathrm{~m}$ sample by Brown, Funfschilling, et al. (2005) revealed that the correction increases with $L$. Arbitrarily assuming a power-law dependence $a=a_{0} L^{x_{a}}$ and $b=b_{0} L^{x_{b}}$, an extrapolation to $L=1 \mathrm{~m}$ yielded $a=0.221$ and $b=0.264$, and via Eq. (29) led to the down-pointing triangles. We consider the up-pointing and down-pointing triangles to be estimates of lower and upper bounds on the actual $\mathrm{Nu}_{\infty}$. Arbitrarily adjusting $a$ and $b$ to the intermediate values 0.25 and 0.32 , respectively, yielded the solid diamonds which are consistent with the data from the $L=0.5 \mathrm{~m}$ sample. New measurements in this very large cell with $\mathrm{Al}$ plates, which together with the $\mathrm{Cu}$-plate data will yield better values of $a$ and $b$, are anxiously awaited.

The solid line in Fig. 4 is the GL prediction (Grossmann and Lohse, 2001). It gives the shape of the experimentally found $\mathrm{Nu}(\mathrm{Ra})$ very well for $\mathrm{Ra} \leq 10^{10}$. For larger Ra the data suggest $\gamma_{\text {eff }}=1 / 3$ whereas the model only reaches such a value for $\gamma_{\text {eff }}$ as $\mathrm{Ra} \rightarrow \infty$ where the model is no longer expected to be applicable.

A detailed discussion of a number of other measurements for $\operatorname{Pr}=\mathcal{O}(1)$ and $\Gamma=0.5$ or 1 (Niemela et al., 2000a; Xu et al., 2000; Ahlers and Xu, 2001; Chavanne et al., 2001; Fleischer and Goldstein, 2002; Roche et al., 2002, 2004; Niemela and Sreenivasan, 2003a; Nikolaenko and Ahlers, 2003; Nikolaenko et al., 2005; Sun, Ren, et al., 2005) is beyond the scope of this review, although we re-visit a few of them in Sec. III.G. We refer the reader to publications by Niemela and Sreenivasan (2003a) and Nikolaenko et al. (2005) where many data sets have been compared. There is excellent agreement between several of them; however, in the range $\mathrm{Ra}$ $\lesssim 10^{12}$ there are differences of up to $20 \%$ or so between some of them. It is not clear whether the origin of these differences is to be found in experimental uncertainties, perhaps associated with wall or plate corrections or other experimental effects, or, as suggested by Niemela and Sreenivasan (2003a), in genuine differences of the fluid dynamics of the various samples. We find the latter explanation somewhat unlikely because, as discussed in Sec. III.F, the heat transport is determined primarily by boundary layer instabilities and is relatively insensitive to the structure of the LSC.

\section{The Prandtl-number dependence of the Nusselt number}

Fluids with $\operatorname{Pr}>1$ are plentiful in the form of various liquids, although accurate determinations of $\mathrm{Nu}(\mathrm{Ra})$ are in many cases problematic because the required physical properties are not known well enough. Typical gases not too close to the critical point have $\operatorname{Pr}=\mathcal{O}(1)$. The range $\operatorname{Pr} \lesssim 0.7$ is difficult to access because most ordinary fluids have $\operatorname{Pr}$ greater than or close to the hard-sphere-gas value 2/3 [see, for instance, Hirschfelder et al. (1964)]. Liquid metals, by virtue of the electronic contribution to the thermal conductivity, have $\operatorname{Pr}=\mathcal{O}\left(10^{-2}\right)$ or smaller, leaving a wide gap in the range from $10^{-2}$ to 0.7 . For the liquid metals it is difficult to obtain very large values of $\mathrm{Ra}$ because the large thermal conductivity requires large heat currents and tends to yield small Rayleigh numbers unless very large samples are constructed. Another problem for liquid metals (see Sec. III.B) is the uncertainty introduced by a large plate correction; however, sidewall corrections should be negligible.

In spite of these difficulties, several researchers attempted low-Pr measurements of $\mathrm{Nu}$, in order to study the $\operatorname{Pr}$ dependence. Measurements with mercury (Pr $=0.025)$ were done by Rossby (1969) $\left(2 \times 10^{4} \leqslant \mathrm{Ra} \leqslant 5\right.$ $\times 10^{5}$ ), by Takeshita et al. (1996) and Naert et al. (1997) 

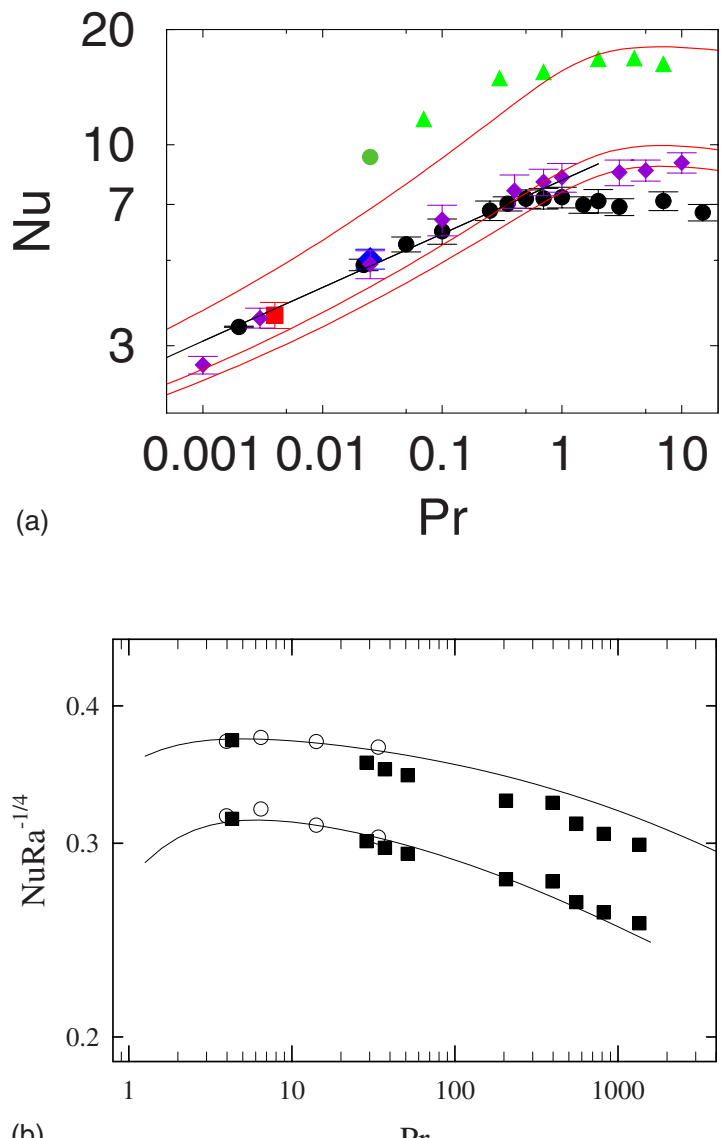

(b)

$\operatorname{Pr}$

FIG. 5. (Color online) Nusselt number versus Prandtl number. (a) $\mathrm{Nu}(\mathrm{Pr})$ for $\mathrm{Ra}=6 \times 10^{5}$ and $\Gamma=1$ from numerical simulations by Verzicco and Camussi (1999) (circles), from experiments with mercury by Rossby (1969) (diamond), and from the experiments with sodium by Horanyi et al. (1999) (square). The straight solid line is a fit to the numerical data with $\operatorname{Pr}<1$ (Verzicco and Camussi, 1999), giving $\mathrm{Nu}=8.1 \mathrm{Pr}^{0.14 \pm 0.02}$. The exponent is in agreement with the low-Pr expectation 1/8 of the GL theory. The upper triangles are the numerical data for $\mathrm{Ra}=10^{7}$ by Kerr and Herring (2000), the dark circle results from the experimental data of Cioni et al. (1997) for the same $\mathrm{Ra}=10^{7}$, and the diamonds are numerical results for $\mathrm{Ra}=10^{6}$ by Breuer et al. (2004). The three solid lines are the results from the GL theory equations (21) and (22) for the three Rayleigh numbers of the numerical data sets, namely, $\mathrm{Ra}=6 \times 10^{5}$, $\mathrm{Ra}=10^{6}$, and $\mathrm{Ra}=10^{7}$, bottom to top. (b) The reduced Nusselt number $\mathrm{NuRa}^{-1 / 4}$ as a function of the Prandtl number for the two Rayleigh numbers $1.78 \times 10^{9}$ (upper set) and $1.78 \times 10^{7}$ (lower set) in the large-Pr regime. Open circles, Ahlers and $\mathrm{Xu}$ (2001). Solid symbols, Xia et al. (2002). Various organic fluids were used. From Xia et al., 2002.

$\left(10^{5} \leqslant \mathrm{Ra} \leqslant 10^{9}\right)$, by Cioni et al. $(1995,1996,1997)$ $\left.\times 10^{6} \leqslant \mathrm{Ra} \leqslant 2 \times 10^{9}\right)$, and by Glazier et al. (1999) (2 $\left.\times 10^{5} \leqslant \mathrm{Ra} \leqslant 8 \times 10^{10}\right)$. Horanyi et al. (1999) made measurements with liquid sodium $\left(\operatorname{Pr}=0.005, \mathrm{Ra} \leqslant 10^{6}\right)$. Together with the results for helium gas, air $(\operatorname{Pr}=0.7)$, and water $(4 \leqslant \operatorname{Pr} \leqslant 7)$, these low-Pr data imply a strong increase of $\mathrm{Nu}$ with $\mathrm{Pr}$ at constant $\mathrm{Ra}$, as shown in Fig. 5(a). For Pr larger than about 1 a saturation sets in and $\mathrm{Nu}$ becomes Pr independent for some Pr range. Recent results using helium gas at low temperatures (Roche et al., 2002) and covering the range $0.7 \lessgtr \operatorname{Pr} \lesssim 21$ suggest a very mild, if any, increase with Pr. Results obtained with various organic fluids (Ahlers and Xu, 2001; Xia et al., 2002) for $\mathrm{Ra}=1.78 \times 10^{9}$ and $1.78 \times 10^{7}$ are shown in Fig. 5 , bottom, and indicate a maximum in $\mathrm{Nu}(\mathrm{Pr})$ near $\mathrm{Pr}$ $\simeq 3$, followed by a very gradual decrease of $\mathrm{Nu}$ with $\mathrm{Pr}$ that can be described by $\mathrm{Nu} \propto \operatorname{Pr}^{-0.03}$ over the $\mathrm{Pr}$ range of the experiments.

One of the successes of the GL model is that it contains most of the features of $\mathrm{Nu}(\mathrm{Pr})$ observed in experiment. When $\mathrm{Ra}$ is not too large, it predicts $\mathrm{Nu} \sim \operatorname{Pr}^{1 / 8}$ at constant $\operatorname{Ra}$ for $\operatorname{Pr} \lesssim 1$, a maximum near $\operatorname{Pr}=3$, and the very gradual decline for larger Pr. For large Pr the GL prediction is shown by the solid lines in Fig. 5(b). Although the parameters of the model had been adjusted using data for Pr up to about 30 (including the open circles in the figure), the model agrees with the measurements up to $\operatorname{Pr} \simeq 2000$. The large Pr behavior resulting from the GL theory has been discussed by Grossmann and Lohse (2001) and the small Pr behavior by Grossmann and Lohse (2008).

\section{E. The aspect-ratio dependence of the Nusselt number}

Several experiments (Wu and Libchaber, 1992; Xu et al., 2000; Ahlers and Xu, 2001; Fleischer and Goldstein, 2002; Funfschilling et al., 2005; Nikolaenko et al., 2005; Sun, Ren, et al., 2005; Niemela and Sreenivasan, 2006a) have probed the dependence of $\mathrm{Nu}$ at constant $\mathrm{Ra}$ and $\operatorname{Pr}$ on $\Gamma$. Using water with $\operatorname{Pr} \simeq 4$, it is found for $\Gamma \lesssim 5$ that $\mathrm{Nu}$ increases, albeit only very slightly, with decreasing $\Gamma$. For larger $\Gamma$ the measurements up to $\Gamma=20$ suggest no further change, indicating that a large- $\Gamma$ regime may have been reached. The weak $\Gamma$ dependence suggests an insensitivity to the nature of the LSC (see also Sec. III.F), which surely changes as $\Gamma$ increases well beyond 1 , and is consistent with the determination of $\mathrm{Nu}$ by instabilities of the thermal BLs. Theoretical efforts to understand the influence of $\Gamma$ on $\mathrm{Nu}$ have been quite limited; see, e.g., Grossmann and Lohse (2003) and Ching and Tam (2006).

\section{F. The insensitivity of the Nusselt number to the LSC}

Several experiments suggest that the Nusselt number in the $\mathrm{Ra}$ range below the transition to the ultimate regime is insensitive to the strength and structure of the LSC. Cioni et al. (1996) measured $\mathrm{Nu}(\mathrm{Ra})$ with a sample of water with $\operatorname{Pr} \simeq 3$ in a container of rectangular cross section in which the azimuthal LSC orientation was more or less fixed. They determined the heat flux both of the original water samples and of the same 
samples after several vertically positioned screens had been installed within them. In the absence of screens shadowgraph visualizations showed that plumes generated at the bottom boundary layer were swept laterally just above the boundary layer by a LSC. The plumes rose vertically in the presence of screens, suggesting a dramatically altered and much weaker LSC. For both cases the heat current was the same within a few percent. This experiment suggests that the heat current is determined primarily by the conductance and instability of the thermal boundary layers which are not influenced significantly by the LSC, and that the plumes with their excess enthalpy will find their way to the top one way or another regardless of any LSC. Cioni et al. (1996) also found that tilting their cells relative to gravity by an angle $\alpha$ as large as $0.06 \mathrm{rad}$, which enhances the Reynolds number of the LSC, had no influence on the heat transport within their resolution of a few percent.

More recently Ahlers, Brown, and Nikolaenko (2006) measured $\mathrm{Nu}(\mathrm{Ra})$ for a cylindrical water sample with $\Gamma$ $=1$ and $\operatorname{Pr}=4.4$ as a function of the tilt angle $\alpha$ with a precision of $0.1 \%$. They found, for example, a very small reduction, by about $0.4 \%$, for a tilt angle $\alpha=0.12 \mathrm{rad}$. In the same experiment the LSC Reynolds number was determined and found to increase by about $25 \%$ for $\mathrm{Ra}$ $=10^{9}$ and by about $12 \%$ for $\mathrm{Ra}=10^{11}$. If the Reynolds number had any direct influence on $\mathrm{Nu}$, one would have expected an increase of $\mathrm{Nu}$ with $\mathrm{Re}$. Again one is led to conclude that the heat transport is independent of the vigor of the LSC and thus presumably determined by LSC-independent boundary layer properties. This finding seems to be in conflict with the final GL results equations (21) and (22), in which $\mathrm{Nu}$ and Re are intimately coupled to each other.

For a $\Gamma=0.5$ water sample Chillà et al. (2004a) measured a reduction of $\mathrm{Nu}$ by about $5 \%$ when they tilted their system by about $0.03 \mathrm{rad}$. Samples of this aspect ratio are more complex because the LSC can consist either of a single convection roll or of a more complex structure approximated by two rolls stacked one above the other (Verzicco and Camussi, 2003; Xi and Xia, 2008b). They conjectured that the tilt stabilizes the single-roll structure, and that this structure gives a smaller heat transport than the two-roll structure, thus accounting for the reduction of Nu. However, it seems surprising to us that for $\Gamma=0.5$ the Nusselt number should be more sensitive to the LSC than it is for the $\Gamma=1$ system.

More evidence for the insensitivity of $\mathrm{Nu}$ to changes in the LSC has been given by Xia and Lui (1997), who altered the LSC into an oscillating four-roll flow pattern by placing staggered fingers on the sidewall and found that Nu changed very little. Xia and Qiu (1999) made an even stronger perturbation to the system by placing a baffle at the cell's mid-height, again finding insensitivity of $\mathrm{Nu}$.

In addition to the evidence of the insensitivity of $\mathrm{Nu}(\mathrm{Ra})$ to changes in the LSC, there is good evidence for the sensitivity of $\mathrm{Nu}(\mathrm{Ra})$ to the structure of the thermal BLs. This is provided by an experiment of $\mathrm{Du}$ and
Tong $(2000,2001)$ who covered the top and bottom plates with triangular grooves that were much deeper than the BL thickness. They found an enhancement of $\mathrm{Nu}(\mathrm{Ra})$ by as much as $76 \%$, with no significant change in the dependence on Ra [see also Ciliberto and Laroche (1999)]. Flow visualization revealed an increase of plume shedding by the protrusions as the mechanism of the $\mathrm{Nu}$ enhancement. Similar results were found by Stringano and Verzicco (2006) in their numerical simulations of RB convection over grooved plates.

\section{G. The dependence of $\mathrm{Nu}$ on $\mathrm{Ra}$ at very large $\mathrm{Ra}$}

Below the transition to the ultimate regime the Nusselt number is determined essentially by properties of the top and bottom thermal boundary layers (see Sec. III.F). As discussed in Secs. II.B and II.C, this is expected to change dramatically in a critical range around some $\mathrm{Ra}^{*}$, defined by the condition that the shear across the laminar (albeit fluctuating) kinetic BL due to the LSC becomes so large that a transition to turbulence is induced within it. Note that the exact value of $\mathrm{Ra}^{*}$ depends on the strength and type of the turbulent noise that perturbs the BLs, but the transition is expected to happen once the shear Reynolds number $\mathrm{Re}_{s}$, based on the kinetic $\mathrm{BL}$ thickness, exceeds $\mathrm{Re}_{s}^{*}=\mathcal{O}(400)$. For $\Gamma=1$ estimates of $\mathrm{Ra}^{*}$ based on the GL theory (Grossmann and Lohse, 2002) and corresponding to $\mathrm{Re}_{s}^{*}=440$ and 220 are shown in Fig. 6(b) as dotted and dashed lines, respectively (since the parameters of the GL theory have been determined only for $\Gamma=1$, an equivalent prediction of $\mathrm{Ra}^{*}$ for general $\Gamma$ unfortunately is not available). These estimates are based on the assumption that a LSC continues to exist at these very large Rayleigh numbers. If it does not, then the transition should eventually be triggered by a destruction of the kinetic BL by turbulent fluctuations rather than by a laminar (albeit fluctuating) flow across the plates. Understanding the regime above $\mathrm{Ra}^{*}$ is of particular importance because it is believed by many to be the asymptotic regime that permits, in principle, an extrapolation to arbitrarily large values of Ra, including those of astrophysical and geophysical interest.

Experimentally it should be possible to observe the predicted transition by a dramatic change in the magnitude and/or the Rayleigh-number dependence of the Nusselt number. For $\mathrm{Nu}(\mathrm{Ra})$ one expects a change from an effective power law with $\gamma_{\text {eff }} \simeq 0.32$ as observed below $\mathrm{Ra}^{*}$ to $\gamma_{\mathrm{eff}} \simeq 0.4$, which due to the logarithmic corrections is somewhat below the predicted asymptotic value $\gamma=1 / 2$ (see Sec. II.C). Another dramatic change, according to the theory, should be the dependence on Pr. For $\mathrm{Ra}<\mathrm{Ra}^{*}$ the Nusselt number is essentially independent of $\operatorname{Pr}$ for $\operatorname{Pr} \gtrsim 1$. For $\mathrm{Ra}>\mathrm{Ra}^{*}$ the Kraichnan prediction is $\mathrm{Nu} \sim \mathrm{Pr}^{-1 / 4}$ [see Eq. (25)], at least for Pr near 1. However, the GL theory predicts $\mathrm{Nu} \sim \operatorname{Pr}^{1 / 2}$ [see Eq. (27)], so there remains some uncertainty on this issue. Nonetheless, any significant $\operatorname{Pr}$ dependence would lead to a 


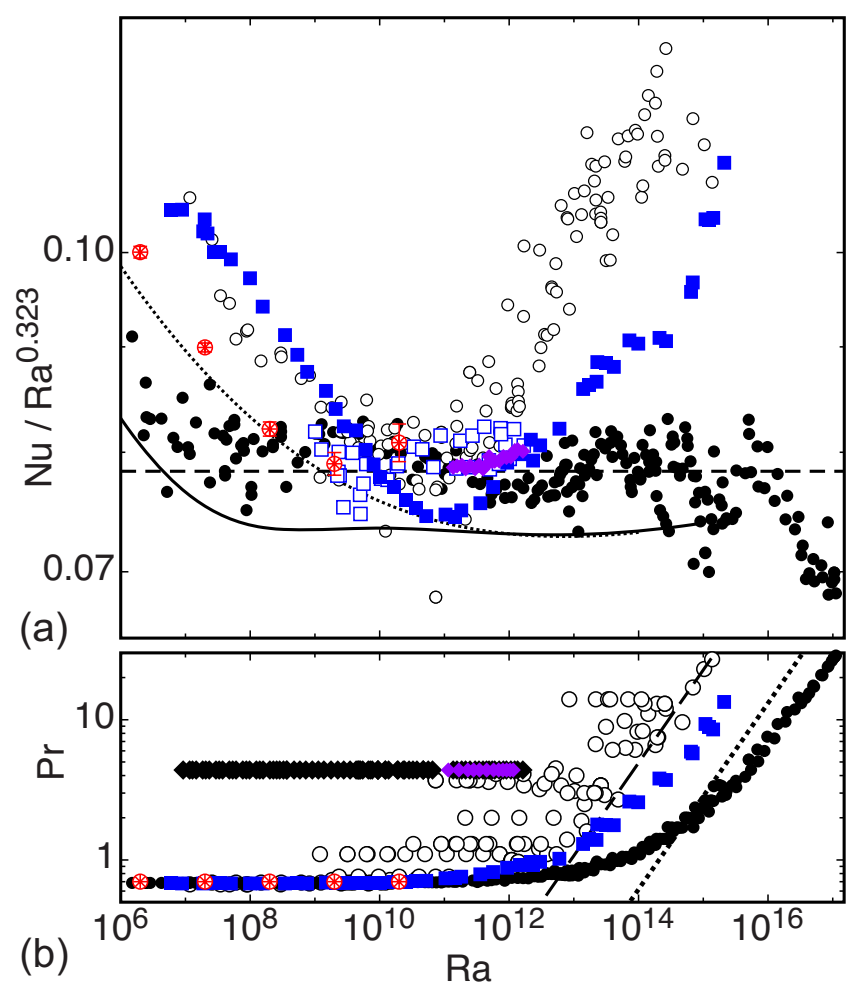

FIG. 6. (Color online) High-precision data comparison of Nusselt versus Rayleigh numbers. (a) $\mathrm{Nu} / \mathrm{Ra}^{\gamma_{\text {eff }}}$ with $\gamma_{\text {eff }}=0.323$ as a function of Ra. Solid circles, $\Gamma=0.5$ (Niemela et al., 2000a) after a correction for plate and sidewall effects (Niemela and Sreenivasan, 2006b). Open circles, $\Gamma=0.5$ (Chavanne et al., 2001). Solid squares, $\Gamma=1$ (Niemela and Sreenivasan, 2003a). Solid diamonds, $\Gamma=0.67$ and 0.43 (Nikolaenko et al., 2005). Open squares, $1 \leqq \Gamma \leqq 3, \operatorname{Pr}=0.7$ (Fleischer and Goldstein, 2002). Stars in circles, numerical results for $\Gamma=1 / 2, \operatorname{Pr}=0.7$ (Stevens et al., 2009) for constant-temperature boundary conditions at the plates. Solid (dotted) line, the GL prediction for $\operatorname{Pr}=0.8(\operatorname{Pr}=29)$. (b) The Prandtl numbers corresponding to the data in (a). In addition, Pr for the measurements for $\Gamma=1$ with water in Fig. 4 are shown as diamonds. The dashed and dotted lines in the bottom figure are estimates of the location of the transition to the Kraichnan regime for $\Gamma=1$, assuming critical boundary layer Reynolds numbers $\operatorname{Re}_{s}^{*}=220$ and 440, respectively (since the parameters of the GL theory have been determined only for $\Gamma=1$, a prediction of $\mathrm{Ra}^{*}$ for smaller $\Gamma$ is not available).

discontinuity of $\mathrm{Nu}(\mathrm{Ra})$ of a size that would depend on Pr.

From Fig. 6(b), one sees that the measurements with water at $\operatorname{Pr}=4.4$ and $\Gamma=1,0.67$, and 0.43 have not reached the regime above $\mathrm{Ra}^{*}$ predicted for $\Gamma=1$. As expected, the measurements for $\Gamma=0.67$ and 0.43 shown in Fig. 6(a), as well as those for $\Gamma=1$ shown in Fig. 4, give no indication of the BL-turbulence transition. Neither do other measurements for $\operatorname{Pr} \approx 4, \Gamma$ between 0.67 and 20, and $\mathrm{Ra}$ up to $5 \times 10^{12}$ (Sun, Ren, et al., 2005).

Measurements using cryogenic helium by Niemela $e t$ al. (2000a) for $\Gamma=0.5$ are shown in Fig. 6, top, as solid circles. The data were corrected recently by some of the original authors (Niemela and Sreenivasan, 2006a) for sidewall and plate effects (see Sec. III.B). ${ }^{2}$ The data are for Rayleigh numbers as large as $10^{17}$, and for Ra up to about $10^{12}$ they are for $\operatorname{Pr} \simeq 0.7$. In the $\mathrm{Ra}$ range of overlap, they are in excellent agreement with the water measurements for $\operatorname{Pr}=4.4$ and $\Gamma=0.67$ and 0.43 , and with the results for compressed gases (Fleischer and Goldstein, 2002) with $\operatorname{Pr} \simeq 0.7,1 \leqslant \Gamma \leqslant 3$, and $1 \times 10^{9} \leq \operatorname{Ra} \leqslant 2 \times 10^{12}$, demonstrating again the insensitivity of $\mathrm{Nu}$ to $\mathrm{Pr}$ and $\Gamma$ in this Ra range, as well as a consistency between the cryogenic and room-temperature experiments. At large Ra the values of Pr for the Niemela et al. (2000a) data increased because of the proximity to the critical point. As shown in Fig. 6(b), the $\Gamma=0.5$ data might have been expected to cross $\mathrm{Ra}^{*}$ somewhere near $\mathrm{Ra}=10^{13}$ or $10^{14}$, but apparently did not do so since they reveal no change of the dependence of $\mathrm{Nu}$ on $\mathrm{Ra}$. Two possible explanations come to mind. Perhaps the LSC was less vigorous in this experiment than it was for $\Gamma=1$. In that case the expected $\mathrm{Re}_{s}^{*} \simeq 400$ would only be reached at even higher Ra. Alternatively, at the very high Ra the LSC may have deteriorated into an unrecognizable entity consisting essentially only of vigorous fluctuations as suggested by Sreenivasan et al. (2002). In that case the GL estimate for $\mathrm{Ra}^{*}$ would no longer be quantitatively applicable.

Within their experimental uncertainty and over the very wide range $10^{7} \leq \mathrm{Ra} \leq 5 \times 10^{15}$ the data of Niemela et al. (2000a) can be described by a single power law $\mathrm{Nu}=N_{0} \mathrm{Ra}^{\gamma_{\text {eff }}}$ with an effective exponent $\gamma_{\text {eff }}=0.323$ and $N_{0}=0.0783$. This power law agrees well with a fit to the data of Fleischer and Goldstein (2002) which (over their much more narrow range of Ra) yielded $N_{0}=0.0714$ and $\gamma_{\mathrm{eff}}=0.327$. Both sets of measurements are inconsistent with an exponent of $1 / 3$. However, they are remarkably consistent with the prediction of GL, which is shown by the solid line in the figure. The drop below the power law for $\mathrm{Ra} \gtrsim 5 \times 10^{15}$ is unexplained. One might have attributed it to non-Boussinesq effects, but for gases near the critical point these would cause an increase of $\mathrm{Nu}$ and not a decrease; see Ahlers et al. (2008) and Sec. VII. Alternatively, one might look at the variation of Pr as an explanation, but in the GL model $\mathrm{Nu}$ is essentially independent of Pr at these large Ra; cf. regime $\mathrm{IV}_{u}$ in Sec. II.B.

An earlier set of data using helium at low temperatures and $\Gamma=0.5$ was obtained by Chavanne et al. (1996, 1997, 2001). It extends up to $\mathrm{Ra} \simeq 10^{15}$, and the results listed by Chavanne et al. (2001) are shown as open circles in Fig. 6. In the range $10^{10} \leqslant \mathrm{Ra} \leqslant 10^{11}$ they agree very well with the other data shown in the figure. For smaller $\mathrm{Ra}$ they are higher than the Niemela et al.

\footnotetext{
${ }^{2}$ Interestingly, this sidewall correction, which was based on the model of Roche, Castaing, Chabaud, Hebral, et al. (2001), yielded corrected data that are nearly identical to those that had been obtained using model 1 of Ahlers (2000) and shown in Fig. 5 of that reference. The plate-effect corrections are quite small for the cryogenic data and have little influence on the interpretation of the data.
} 
(2000a) data. A possible reason might be found in a difference of the sidewall correction that was applied. ${ }^{3}$ More interesting is the difference between the two data sets that evolves as $\mathrm{Ra}$ grows beyond $10^{11}$. In that regime the open circles in the figure can be described within their scatter by a power law with $\gamma_{\mathrm{eff}} \simeq 0.38$. Chavanne et al. (1997) interpret this result as corresponding to the expected $\gamma=1 / 2$ in the Kraichnan regime, modified by the logarithmic corrections that are attributable to a viscous sublayer. Thus they claim to have entered the "ultimate," or asymptotic, regime of turbulent RB convection (Chavanne et al., 1997). However, the transition at $\mathrm{Ra}^{*}$ just above $10^{11}$ is lower than the theoretical estimates for the shear-flow boundary layer instability. For $\operatorname{Pr}=1$ and $\mathrm{Ra}=3 \times 10^{11}$ the GL model yields (in the $\Gamma=1$ case) $\mathrm{Re}_{s} \simeq 100$, which is too low for a shearinduced transition to turbulence in the boundary layer. An explanation in terms of a shear-induced BL transition would require a more vigorous LSC for the $\Gamma=0.5$ case than was measured (see Sec. IV) for the $\Gamma=1.0$ case. ${ }^{4}$ In any case, the data of Chavanne et al. (2001), and the interpretation in terms of a transition to the Kraichnan regime, differ dramatically from the measurements of Niemela et al. (2000a) who did not find this transition even though their data extend to higher values of $\mathrm{Ra}$, and were done at somewhat lower Pr where the shear transition should occur at even smaller $\mathrm{Ra}$. The reason for this difference remains unresolved at this time, and the resolution of this apparent conflict between the two data sets is one of the major challenges in this field of research.

Yet another set of data, shown as solid squares in Fig. 6(a), was obtained with low-temperature helium by $\mathrm{Ni}$ emela and Sreenivasan (2003a), using the original apparatus of the $\Gamma=0.5$ measurements by Niemela et al. (2000a), but with a sample of reduced height that had $\Gamma=1.0$. Unfortunately these results do not help to clarify the situation. They are consistent with other data in the $\mathrm{Ra}$ range near $10^{11}$. At smaller Ra they agree fairly well with the Chavanne et al. (2001) data, but differ from the sidewall-corrected Niemela et al. (2000a) data. Here again one would be tempted to invoke the sidewall effect as a possible explanation. More difficult to disregard are the data for $\mathrm{Ra} \gtrsim 10^{12}$, where sidewall corrections are

\footnotetext{
${ }^{3}$ In fact, for $\mathrm{Ra}<10^{9}$ the data from Niemela et al. (2000a) before their sidewall correction were much closer to the data from Chavanne et al. (2001) than after this correction was made.

${ }^{4}$ Chavanne et al. (2001) measured the vertical LSC velocity component $v_{z}(r, z, \theta)$ in the horizontal midplane $(z=0)$ and at a radial position $r=L / 4$ halfway between the center line and sidewall. The significant size found for $v_{z}$ suggests the existence of well developed upflow and/or downflow in the midplane, implying that the LSC consisted of a single convection roll rather than of two rolls one above the other. The measurement yielded a Reynolds number that is not very different from that for the $\Gamma=1$ case (see Sec. IV), but it is difficult to know precisely the corresponding shear across the viscous BLs at the top and bottom plates.
}

negligible. There the data fall between the two $\Gamma=0.5$ cryogenic data sets, thus adding to the complexity of experimental information about a possible Kraichnan transition.

The Grenoble-Lyon group undertook several investigations in an attempt to find an explanation for the differences between the various data sets for $\mathrm{Ra} \gtrsim 10^{12}$. For instance, Chillà et al. (2004b) developed a model that attempted to explain the difference in terms of a finite plate-conductivity effect (see Sec. III.B); but measurements with relatively low-conductivity brass plates by Roche et al. (2005) yielded results comparable to the high-conductivity copper-plate results. In a separate experiment Roche, Castaing, Chabaud, and Hebral (2001) made measurements using helium in a sample cell with $\Gamma=0.5$ with walls and plates that were covered completely by grooves. The depth of the grooves was stated to be less than the thermal boundary layer thickness. Such a geometry is asserted to remove the influence of the sublayer which is responsible for the logarithmic corrections. For $\mathrm{Ra} \gtrsim 10^{12}$ this experiment yielded an exponent quite close to 0.5 , consistent with the expected Kraichnan value of $1 / 2$. However, Niemela and Sreenivasan (2006a) pointed out that the BL thickness decreases with increasing $\mathrm{Ra}$ and becomes comparable to the groove depth in the Ra range of the measurements. In such a case the measurements of Du and Tong (2000) using a sample with grooves in the plates that were deeper than the BL thickness indicate that the prefactor of an effective power law describing $\mathrm{Nu}(\mathrm{Ra})$ increases by as much as $76 \%$ for deep grooves. Thus it was suggested by Niemela and Sreenivasan (2006a) that the results of Roche, Castaing, Chabaud, and Hebral (2001) might possibly be due to a crossover between rough surfaces with a groove depth less than the BL thickness to a regime where the groove depth is larger than the $\mathrm{BL}$ thickness. More work is needed to resolve this issue.

An interesting experiment related to the Kraichnan regime was by Gibert et al. (2006), taking up earlier experiments by Perrier et al. (2002) and experimentally realizing the theoretically suggested homogeneous RB turbulence (Lohse and Toschi, 2003; Calzavarini et al., 2006). Gibert et al. (2006) used a vertical channel with wide entrance and exit sections that avoided the influence of the thermal BLs on Nu. They found relationships for $\mathrm{Nu}$ and $\mathrm{Re}$ (based on the velocity fluctuations) consistent with Eqs. (27) and (28) when they redefined $\mathrm{Ra}$ in terms of an intrinsic $\Delta$-dependent length scale proportional to the ratio of temperature-fluctuation amplitudes and the vertical thermal gradient, instead of using a sample-geometry-dependent and $\Delta$-independent length. The same scaling was also obtained by Cholemari and Arakeri $(2005,2009)$ for buoyancy driven turbulent exchange flow in a vertical pipe. The flow was driven by an unstable density difference across the ends of the pipe, created using brine and distilled water. Away from either end, a fully developed region of turbulence existed with a linear density gradient. With a Rayleigh number based on the local density gradient re- 
lations consistent with Eqs. (27) and (28) were found experimentally.

Also local measurements of the heat flux can result in the $1 / 2$ exponent predicted by Kraichnan (1962). Shang et al. (2008), based on their earlier measurements (Shang et al., 2003, 2004), determined a time-averaged local Nusselt number at fixed positions $\boldsymbol{x}$ and given by

$$
\mathrm{Nu}(\boldsymbol{x})=\left\langle u_{3}(\boldsymbol{x}, t) \theta(\boldsymbol{x}, t)\right\rangle_{t} / \kappa \Delta L^{-1}
$$

in a $\Gamma \approx 1$ cylindrical cell filled with water, in the range $10^{8} \leqq \mathrm{Ra} \lesssim 2 \times 10^{10}$. At half height they found $\mathrm{Nu}(\boldsymbol{x})$ $=1.5 \mathrm{Ra}^{0.24 \pm 0.03}$ when $\boldsymbol{x}$ was close to the sidewall where the heat was primarily transported by plumes, and $\mathrm{Nu}(\boldsymbol{x})=3.5 \times 10^{-4} \mathrm{Ra}^{0.49 \pm 0.03}$ when $\boldsymbol{x}$ was close to the cell center where the plume density was much less. ${ }^{5}$ These two different local scaling laws had been predicted by Grossmann and Lohse (2004); they correspond to the two independent scaling contributions $\epsilon_{\theta, \mathrm{pl}}$ $=\kappa \Delta^{2} L^{-2} \mathrm{Nu}_{\text {side }}$ and $\epsilon_{\theta, \mathrm{bg}}=\kappa \Delta^{2} L^{-2} \mathrm{Nu}_{\text {center }}$ to the thermal dissipation rate $\epsilon_{\theta}=\kappa \Delta^{2} L^{-2} \mathrm{Nu}$ [see Eq. (13)]. The theory predicts that for $\mathrm{Ra} \leq 10^{14}$ the heat transport via the flow close to the sidewall dominates, but around $\mathrm{Ra} \approx 10^{14}$ the heat flux through the center and thus the background fluctuations take over. Interestingly, when extrapolating the measured power laws for the local Nusselt number in the center and close to the sidewall towards larger Ra, Shang et al. (2008) also obtain a crossover around $\mathrm{Ra} \approx 10^{14}$. The spatial inhomogeneity of the local heat flux also has been confirmed by the numerical simulations of Shishkina and Wagner (2007).

\section{EXPERIMENTAL MEASUREMENTS OF THE REYNOLDS NUMBERS}

\section{A. Reynolds numbers based on the large scale convection roll}

The geometrical features and dynamics of the large scale circulation (LSC) depend on the symmetry and aspect ratio of the sample. Here we focus on cylindrical samples with $\Gamma \simeq 1$ because for them the flow geometry is relatively simple, the dynamics is very rich, and the experimental studies are most extensive. Then the flow occurs in a near-vertical plane and yields near-elliptical stream lines, with the long axis of the ellipse slightly tilted relative to gravity [see, for instance, Verzicco and Camussi (1999), Qiu et al. (2004), Sun, Xia, et al. (2005)], as shown in the time-average velocity map Fig. 1(c). Depending on $\mathrm{Ra}$, two small counter-rotating vortices positioned near the corners close to the minor axis

\footnotetext{
${ }^{5}$ Note that in earlier work by Ching et al. (2004) a less steep scaling of $\mathrm{Nu}(\boldsymbol{x})$ with $\mathrm{Ra}$ was derived at the center of the convection cell. One possible source of discrepancy is that the association of the length scale resulting from balancing buoyancy and viscous forces with the thermal boundary layer thickness done in that work does not generally hold.
}

of the ellipse may be more or less prominent. In the 2D numerical simulations of Sugiyama et al. (2009) they can be very pronounced, depending on Ra.

Often it is said that the large scale convection roll is driven by the localized volumes of hot or cold fluid known as plumes that are emitted from the top and bottom thermal BLs as a result of a BL instability, and no doubt these plumes play an important role. But in addition some of the heat current is conducted across the BLs, warming or cooling the fluid adjacent to them. This in itself, without the presence of the plume inhomogeneities, would drive the flow as it does closer to the onset of convection where there are no plumes. Thus presumably the LSC derives its existence from a combination of these two heat-transport mechanisms with the relative importance of each depending on $\mathrm{Pr}$ and $\mathrm{Ra}$, and indeed it seems difficult to separate one from the other. For $\mathrm{Pr} \simeq 4$ it is known that, away from the BLs, this heat current leads to a destabilizing timeaveraged vertical gradient of the azimuthal average of the temperature which is strongest near the sidewall while the interior is more nearly isothermal (Brown and Ahlers, 2007b). Superimposed upon this azimuthally averaged gradient is the warm upwelling and cold downwelling current of the LSC which, when time averaged, leads to a near-sinusoidal azimuthal temperature variation with period $2 \pi$ and amplitude $\delta$ at the side wall (Brown and Ahlers, 2007b), and a near-linear temperature variation along a diameter (Qiu and Tong, 2001b).

Although the speed of the flow shown in Fig. 7(a) varies considerably with position, one might expect that the large central roll can be described by a unique turnover time $\mathcal{T}$. Using $L$ as a relevant length scale, one can define a Reynolds number

$$
\operatorname{Re}^{\mathrm{LSC}} \equiv \frac{2 L^{2}}{\mathcal{T}_{\nu}}
$$

We would not expect $\mathrm{Re}^{\mathrm{LSC}}$ to describe all aspects of the flow field; for instance, the small counter-rotating vortices might require a different Reynolds number which might even have a different dependence on Ra; but the main features, for instance, those predicted by the GL model, might be related to $\mathrm{Re}^{\mathrm{LSC}}$. Further, it has been suggested that the actual path length of the LSC circulation varies with $\mathrm{Ra}$ because the shape of the flow field changes; this feature would introduce an additional $\mathrm{Ra}$ dependence of $\mathrm{Re}^{\mathrm{LSC}}$ because the length scale, set equal to $2 L$ in Eq. (31), would no longer be a constant (Niemela and Sreenivasan, 2003b; Sun and Xia, 2005).

To our knowledge there are no direct measurements of $\mathcal{T}$. However, $\mathcal{T}$ was inferred from local measurements of various velocity components [see Fig. 7(a) for an example] and the assumption of a constant circulation path length proportional to $L$. The time-averaged maximum vertical velocity component $v_{\max }$ (Qiu and Tong, 2001a; Lam et al., 2002) near the region between the viscous boundary layers and the bulk of the system [sometimes known as the "mixing zone" (Castaing et al., 1989)] gave

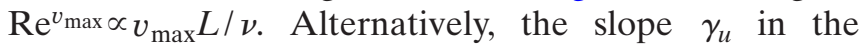



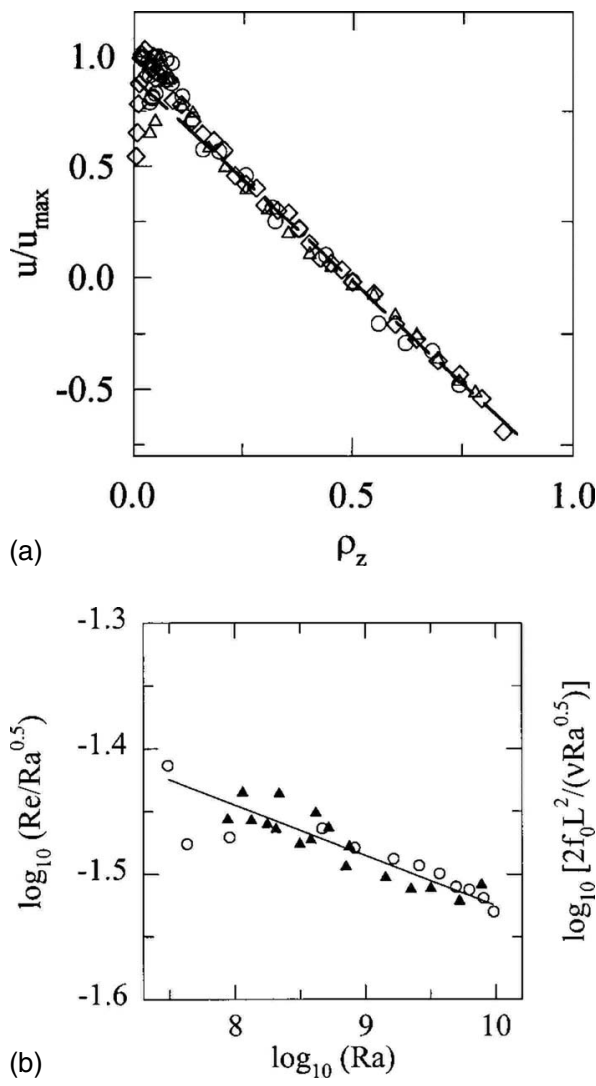

FIG. 7. Profile and scaling of velocity. (a) Normalized horizontal velocity $u / u_{\max }$ as a function of the vertical position $\rho_{z}$ $=z / L$. The measurements were made at $\mathrm{Ra}=3.08 \times 10^{7}$ (circles), $9.14 \times 10^{7}$ (triangles), and $3.7 \times 10^{9}$ (diamonds). From Qiu and Tong, 2001b. Note that the data extend only up to $\rho_{z} \simeq 0.85$; thus the minimum near the sample top is not shown. (b) Reduced Reynolds numbers $\mathrm{Re}^{u} / \mathrm{Ra}^{1 / 2}$ (circles) and $\mathrm{Re}^{\omega} / \mathrm{Ra}^{1 / 2}$ (solid triangles), defined in the text, as functions of $\mathrm{Ra}$. The measurements were done in water at $\operatorname{Pr} \sim 5.5$. From Qiu and Tong, 2002.

sample interior away from the mixing zone of the timeaveraged horizontal component $u$ of the LSC velocity as a function of the vertical position along the sample axis [see Fig. 7(a)] was used to define and determine $\mathrm{Re}^{u}$ $=\gamma_{u} L^{2} / \nu$ (Qiu and Tong, 2001a, 2002) [open circles in Fig. 7(b)].

An interesting property of the LSC is a torsional azimuthal oscillation mode with frequency $f_{0}=\omega_{0} / 2 \pi$ (Funfschilling and Ahlers, 2004; Funfschilling et al., 2008) that can be used to define $\operatorname{Re}^{\omega} \equiv 2 L^{2} f_{0} / \nu$. Well before the spatial nature of this mode was known, its frequency was measured in numerous single-point determinations of the temperature or the velocity (Heslot et al., 1987; Castaing et al., 1989; Ciliberto et al., 1996; Takeshita et al., 1996; Xin et al., 1996; Cioni et al., 1997; Xin and Xia, 1997; Qiu et al., 2000, 2004; Qiu and Tong, 2001a, 2001b, 2002; Lam et al., 2002), both of which have an oscillatory contribution provided the probe is not located in the horizontal midplane of the sample where the amplitude of this mode vanishes (Funfschilling et al., 2008). Some of the single-point measurements yielded results for $\mathrm{Re}^{\omega}$ that were equal to $\mathrm{Re}^{u}$ within experimental resolution (Qiu and Tong, 2002), as shown in Fig. 7(b). The reason for this equality is not known at this time. Some other experimental investigations indicated that there is a distinct difference between the Ra dependence of $\operatorname{Re}^{u_{\max }}$ and $\operatorname{Re}^{\omega}$ (Lam et al., 2002) and that these Ra dependences change with Pr. Clearly there remain some unresolved issues. ${ }^{6}$

\section{B. Reynolds numbers based on plume motion}

An estimate of another Re was based on the motion of plumes. When a cold or warm plume passes a local temperature probe, it produces a positive or negative deviation of the local temperature from the mean. The local time-averaged vertical plume speed $v_{\mathrm{pl}}=l / t_{\mathrm{pl}}$ was thus determined from the peak location $t_{\mathrm{pl}}$ of time crosscorrelation functions between two temperatures measured with probes separated vertically by a small distance $l$ (Castaing et al., 1989; Sano et al., 1989; Takeshita et al., 1996; Chavanne et al., 1997, 2001; Niemela et al.,

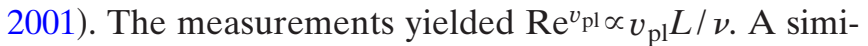
lar technique used thermometers mounted in the sidewall at the horizontal midplane. A single temperature sensor yielded a time autocorrelation (AC) function with a broad peak at a delay time corresponding to the plume turnover time $\mathcal{T}_{\mathrm{pl}}$ (Ahlers, Brown, and Nikolaenko, 2006; Brown et al., 2007). Similarly, two sensors on opposite sides of the sample yielded time cross-correlation (CC) functions with broad peaks corresponding to half the plume turnover time $\mathcal{T}_{\mathrm{pl}} / 2$. These measurements gave Reynolds numbers $\mathrm{Re}^{\mathrm{pl}} \equiv\left(2 L / \mathcal{T}_{\mathrm{pl}}\right) L / \nu$. Quite remarkably, measurements indicate over a wide parameter range that $\mathrm{Re}^{v_{\max }}=\mathrm{Re}^{u}=\mathrm{Re}^{\mathrm{pl}}=\mathrm{Re}^{v_{\mathrm{pl}}}$ within fairly small experimental errors. This can be interpreted to mean that the plume circulation is slaved to the LSC, or vice versa, and that all of these quantities (where they agree with each other) yield a reliable representation of $\mathrm{Re}^{\mathrm{LSC}}$.

The cross-correlation functions for two thermometers at the midplane but on opposite sides had extrema that were negative, indicting a correlation between the warm rising plumes on one side and the cold falling plumes on the other side. This tends to support the ideas of Villermaux (1995), who suggested that a plume impinging on the BL causes an instability and an associated emission of a new plume of the opposite type. However, the width of the cross-correlation function extremum, which was not very different from $\mathcal{T}_{\mathrm{pl}}$, indicates that this is not a periodic process as originally suggested. The periodic signal, when observed in some experiments, presumably

\footnotetext{
${ }^{6}$ Evaluation of experiments is complicated by experimentalists that often slightly tilt their samples so as to obtain a dominant LSC orientation for their measurements. Measurements of $\mathrm{Re}$ are sensitive to the sample alignment relative to gravity (Ahlers, Brown, and Nikolaenko, 2006), and in a tilted sample $f_{0}$ can be observed even at the horizontal midplane.
} 


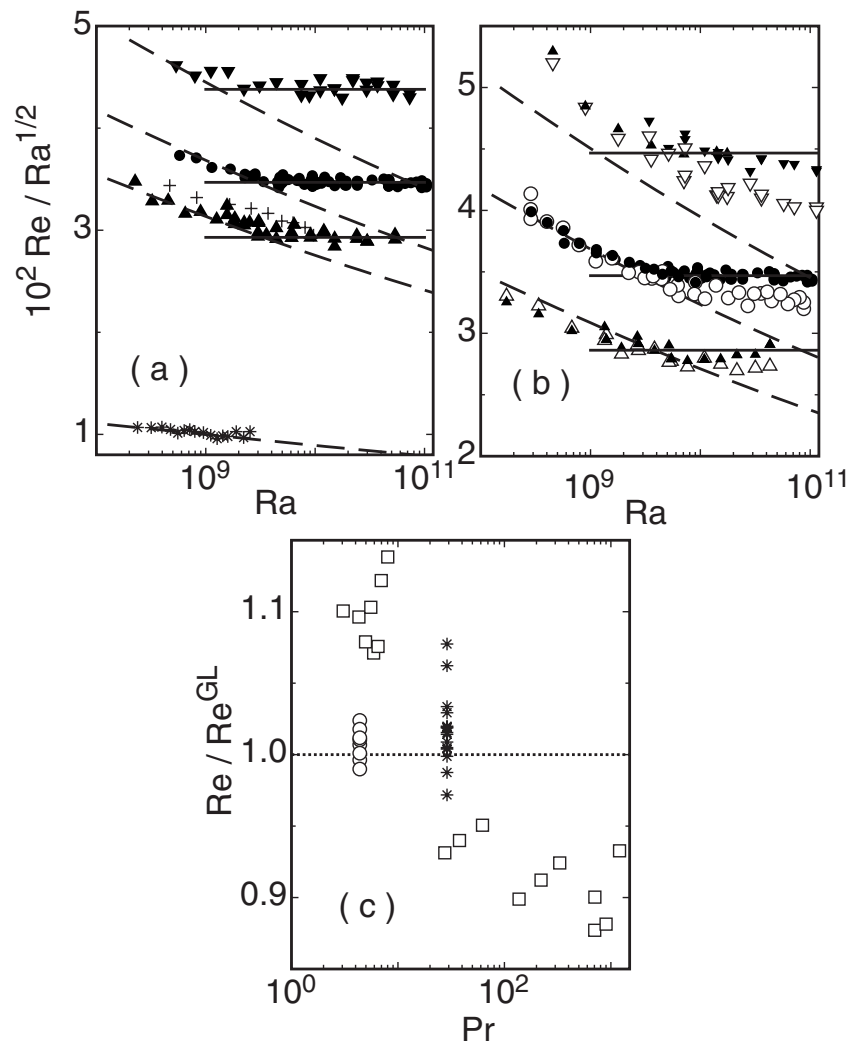

FIG. 8. Parameter dependences of Reynolds number. (a), (b) Reduced Reynolds numbers $\mathrm{Re} / \mathrm{Ra}^{1 / 2}$. (a) $\mathrm{Re}^{\mathrm{pl}}$ for $\operatorname{Pr}=4.38$ (circles), $\operatorname{Pr}=5.55$ (up-pointing triangles), and $\operatorname{Pr}=3.32$ (downpointing triangles), from Brown et al., 2007. Stars, $\mathrm{Re}^{\omega}$ for Pr =28.9, from Brown et al., 2007. Pluses, $\mathrm{Re}^{u}$, from Qiu and Tong, 2002, $\operatorname{Pr} \simeq 5.4$. Dashed lines (from top to bottom), GL predictions for $\mathrm{Pr}=3.32,4.38,5.55$, and 28.9. (b) $\mathrm{Re}^{\mathrm{pl}}$ (solid symbols) and $\mathrm{Re}^{\omega}$ (open symbols) for $\operatorname{Pr}=4.38$ (circles), $\operatorname{Pr}$ $=5.68$ (up-pointing triangles), and $\operatorname{Pr}=3.26$ (down-pointing triangles). Dashed lines (from top to bottom), GL predictions for $\operatorname{Pr}=3.26,4.38$, and 5.68. (c) The ratio of Re to the GL prediction as a function of $\operatorname{Pr}$ for $\mathrm{Ra} \leqslant 3 \times 10^{9}$. Open circles, $\mathrm{Re}^{\mathrm{pl}}$, $\operatorname{Pr}=4.38$. Stars, $\mathrm{Re}^{\omega}, \operatorname{Pr}=29.8$. Squares, $\mathrm{Re}^{\omega}$, from Lam et al., $2002,5.6 \leqslant \operatorname{Pr} \leqslant 1206$.

is due to the torsional oscillation discussed above and in Sec. VIII. ${ }^{7}$

For modest $\mathrm{Ra}$, say $\mathrm{Ra} \leq 3 \times 10^{9}$ when $\mathrm{Pr} \simeq 4$, the GL prediction (Grossmann and Lohse, 2002) is in good agreement with experimental results (Qiu and Tong, 2002; Brown et al., 2007) for $\operatorname{Re}^{u}(\operatorname{Ra}, \operatorname{Pr}), \operatorname{Re}^{\omega}(\operatorname{Ra}, \operatorname{Pr})$, and $\operatorname{Re}^{\mathrm{pl}}(\mathrm{Ra}, \mathrm{Pr})$ (see Fig. 8). In that parameter range all three measured quantities, so far as they have been de-

\footnotetext{
${ }^{7}$ Very recently Xi et al. (2009) observed that the dynamics of the LSC, in addition to the twisting mode discovered by Funfschiling and Ahlers (2004), involves also a "sloshing mode," see Sec. VIII.A. A theoretical explanation of the origin of both modes was given recently by Brown and Ahlers (2009). Rather than coherent plume motion, it is possible that the sloshing mode is responsible for the signal corresponding to $\mathrm{Re}^{\mathrm{pl}}$. In that case the remarks about plume correlation would be inapplicable.
}

termined, agree with each other. In that regime both the prediction and the experiment can be well described by a power law $\mathrm{Re} \propto \mathrm{Ra}^{\gamma_{\text {eff }}}$ with a very slightly $\mathrm{Ra}$ dependent effective exponent $\gamma_{\text {eff }} \simeq 0.44$ [there also are some notable differences between the predictions and some other measurements (Lam et al., 2002)]. However, for $\mathrm{Ra} \geq 3 \times 10^{9}$ the measurements reveal a relatively sudden transition to a new state of the system, with a $\mathrm{Re}^{\mathrm{pl}}$ that is larger than $\mathrm{Re}^{\omega}$, which in turn is larger than the GL prediction. These results agree well with recent experimental results for $\mathrm{Re}^{\mathrm{pl}}$ obtained by Sun and Xia (2005) in the range $7 \times 10^{10} \leqq \mathrm{Ra} \lesssim 2 \times 10^{12}$. If the GL model still correctly predicts the $\mathrm{Ra}$ dependence of $\mathrm{Re}^{\mathrm{LSC}}$ in this parameter range, then the experimental results for $\mathrm{Re}^{\mathrm{pl}}$ and $\mathrm{Re}^{\omega}$ suggest the existence of a new LSC state in which the plumes and the LSC are no longer slaved to each other and where the twist oscillation of the LSC is no longer synchronous with the LSC turnover time [see also Xi et al. (2006)]. The observation that $\mathrm{Re}^{\mathrm{pl}}>\mathrm{Re}^{\mathrm{LSC}}$ would then indicate that the plumes rise and fall more rapidly than the background flow of the LSC. However, Niemela and Sreenivasan (2003a) and Sun and Xia (2005) suggested that it is more likely that the LSC evolves into a more complex flow structure where its dynamics can no longer be described quantitatively by a uniquely defined Reynolds number. It is unclear at present whether the difference between this state and the one at smaller $\mathrm{Ra}$ will be found in the geometry of the flow, in the nature of the viscous boundary layers that interact with it, or in the nature and frequency of plume shedding by the thermal boundary layers adjacent to the top and bottom plates.

The dependence of $\mathrm{Re}^{\omega}$ on $\operatorname{Pr}$ has been investigated over a wide range of Pr by Lam et al. (2002). The data for $\mathrm{Ra} \leqslant 3 \times 10^{9}$ are shown as squares in Fig. 8(c). Their agreement with the GL prediction is not as good as one might wish. However, at $\operatorname{Pr} \simeq 4$, they also differ from the measurements of $\mathrm{Re}^{\mathrm{pl}}$ by Brown et al. (2007). More work over a wide Pr range is desirable.

\section{Nu(Ra,Pr) AND Re(Ra,Pr) IN DIRECT NUMERICAL SIMULATIONS}

Direct numerical simulations (DNS) of RayleighBénard flow have several advantages in comparison to experiments: (i) Any local or global quantity can be "measured" without interfering with the probe or having restricted accessibility. (ii) The boundary conditions can be chosen as in the idealized RB case, i.e., with exactly zero heat flux through the sidewalls and constant temperatures at the top and bottom plates. Therefore a modeling of sidewall and plate corrections (see Sec. III.B) is not necessary. (iii) The material properties of the fluid can be chosen at will. One can study fluids, for example, which exactly obey the Boussinesq approximation, or fluids which show temperature dependence of one material property only. By doing simulations with such fluids, theoretically suggested mechanisms can be tested. 
However, obviously there are also major disadvantages of DNS, the main one being the limitations in the obtainable sizes of $\mathrm{Ra}$ and $\mathrm{Pr}$. For fixed $\mathrm{Pr} \approx 1$ the required CPU time increases roughly as $\mathrm{Ra}^{3 / 2} \ln \mathrm{Ra}$. The spatial resolution requirements for thermal convection have been defined by Grötzbach $(1982,1983)$. In some simulations good small scale resolution, which is particularly crucial in the BLs, is sacrified for higher Ra.

In this DNS part of the review we focus on global properties such as $\mathrm{Nu}$ and $\mathrm{Re}$ and the dynamics of the wind, omitting DNS results for the small scales.

The RB simulations by DeLuca et al. (1990), Werne et al. (1991), and Werne (1993) were restricted to two dimensions and employed free-slip (but impermeable) velocity boundary conditions at the sidewalls, to make efficient use of pseudospectral methods. Note that freeslip sidewall BCs lead to a different large scale circulation (LSC) pattern than no-slip sidewall BCs. In particular, the counter rolls in the corners of the cell are suppressed. With this method, DeLuca et al. (1990) carried out simulations up to $\mathrm{Ra}=1.6 \times 10^{8}$ for $\mathrm{Pr}=1$, finding an effective $\mathrm{Nu}$ vs Ra power-law exponent consistent with $2 / 7$ at the largest Ra. Werne (1993) extended these 2D simulations to $\operatorname{Pr}=7$.

3D simulations in the early 1990s-also employing spectral methods-could only achieve relatively small Ra, e.g., $\mathrm{Ra}=6.5 \times 10^{6}$ in the work by Balachandar et al. (1989) and Sirovich et al. (1989) or $\mathrm{Ra}=6.3 \times 10^{5}$ in the work by Christie and Domaradzki (1992); both are too small to make statements on exponents for power-law dependences on $\mathrm{Ra}$, and also because there are still coherent flow structures.

One of the first 3D RB simulations from which such exponents could be extracted was that by Kerr (1996). $\mathrm{He}$ employed lateral periodic $\mathrm{BCs}$ and achieved $\mathrm{Ra}=2$ $\times 10^{7}$ on a $288 \times 288 \times 96$ grid. For $\mathrm{Pr}=0.7$ he found an effective power law $\mathrm{Nu} \sim \mathrm{Ra}^{0.28}$. Later, Kerr and Herring (2000) extended these simulations to Prandtl numbers in the range $0.07<\operatorname{Pr}<7$, finding $\mathrm{Nu} \sim \mathrm{Ra}^{0.25}$ for the smallest $\operatorname{Pr}$ and effective exponents consistent with $2 / 7$ for $\mathrm{Pr} \geqslant 0.7$. For the Prandtl dependence of $\mathrm{Nu}$ at fixed $\mathrm{Ra}$ $=10^{7}$ and $\operatorname{Pr} \lesssim 0.7$ (see Fig. 5, top), Kerr and Herring (2000) give an effective exponent of 0.12. A more recent example of a spectral RB code is the work of Hartlep et al. (2003), achieving $\mathrm{Ra}=10^{7}$. In that work the focus was on the flow organization in RB convection with sidewise periodic boundary conditions.

A second class of DNS for RB convection is that of lattice-Boltzmann (LB) simulations (Benzi et al., 1994). In one of the first large LB RB simulations Benzi et al. (1998) achieved $\mathrm{Ra} \approx 3.5 \times 10^{7}$ and found $\gamma_{\mathrm{Nu}}$ $=0.283 \pm 0.003$ for the effective $\mathrm{Nu}$ vs Ra power-law exponent (they used $\operatorname{Pr}=1$ and free slip on vertical walls).

The third class of simulations contains DNS based on finite difference or finite volume. Verzicco and Camussi (1999), building on their earlier work (Verzicco and Camussi, 1997; Camussi and Verzicco, 1999), employed such finite-difference simulations to obtain effective power-law exponents for both $\mathrm{Nu}$ and $\mathrm{Re}$ vs $\mathrm{Ra}$ for sev- eral Pr for Ra up to $2 \times 10^{7}$ [see Fig. 5(a)]. The advantage of this method is that the correct no-slip BCs at the sidewall can easily be implemented and that simulations can also be performed efficiently in the cylindrical geometry as used in many experiments, so that a direct comparison with the experimental results is possible. A further advantage of finite-difference or finitevolume methods is that the spatial resolution can easily be refined at will in the BLs, using a coarser grid in the bulk. For the small Prandtl number $\operatorname{Pr}=0.025$ and $\Gamma=1$ the effective power law $\mathrm{Nu} \approx 0.119 \mathrm{Ra}^{0.25}$ was found in the regime $5 \times 10^{4} \leqslant \mathrm{Ra} \leqslant 10^{6}$. For $\operatorname{Pr}=0.7$ Verzicco and Camussi (1999) obtained the effective power law $\mathrm{Nu}$ $\sim \mathrm{Ra}^{0.285}$. They also explored the Pr dependence of $\mathrm{Nu}$ over the range $0.0022 \leqslant \operatorname{Pr} \leqslant 15$ at fixed $\mathrm{Ra}=6 \times 10^{5}$, finding $\mathrm{Nu} \approx 8.5 \mathrm{Pr}^{0.14}$ as an effective power law. For the Reynolds number they found $\operatorname{Re} \sim \operatorname{Pr}^{-0.73}$ for $\operatorname{Pr}<1$ and $\operatorname{Re} \sim \operatorname{Pr}^{-0.94}$ for $\operatorname{Pr}>1$. Similarly, Breuer et al. (2004) performed finite volume integrations of the Boussinesq equations, finding $\mathrm{Nu} \sim \operatorname{Pr}^{0.182 \pm 0.012}$ and $\mathrm{Re}$ $\sim \operatorname{Pr}^{-0.607 \pm 0.012}$ for $10^{-3} \leqslant \operatorname{Pr} \leqslant 1$ and $\mathrm{Nu} \sim \operatorname{Pr}^{0.032 \pm 0.003}$ and $\operatorname{Re} \sim \operatorname{Pr}^{-0.998 \pm 0.014}$ for $1 \leqslant \operatorname{Pr} \leqslant 10^{2}$, for no-slip boundary conditions and $\mathrm{Ra}=10^{6}$.

Verzicco and Camussi (2003) and Stringano and Verzicco (2006) extended the earlier simulations to the remarkably high $\mathrm{Ra}=2 \times 10^{11}$ for a slender $\Gamma=1 / 2$ cell and $\operatorname{Pr}=0.7$. Beyond $\mathrm{Ra}=10^{9}$ the numerical data are consistent with $\mathrm{Nu} \sim \mathrm{Ra}^{1 / 3}$. The focus of those papers is on the flow organization in the $\Gamma=1 / 2$ cell: beyond $10^{10}$ and for $\operatorname{Pr} \approx 0.7$ the single convection roll can break up into two smaller counter-rotating rolls, each approximately of aspect ratio 1. Stringano and Verzicco (2006) in addition showed that the thermal properties of the sidewalls can stabilize the large-scale convection roll.

At present the largest-Ra DNS of RB flow are the ones by Amati et al. (2005) and Verzicco and Sreenivasan (2008), achieving $\mathrm{Ra}=2 \times 10^{14}$ for $\Gamma=1 / 2$ and $\operatorname{Pr}=0.7$, though, as recently shown by Stevens et al. (2009), at the prize of under-resolving the fine structures of the flow, which leads to Nusselt numbers that are too large. Sidewall corrections (Verzicco, 2002) and plate corrections (Verzicco, 2004) have been studied, which were discussed in Sec. III.B.

Another advantage of finite-difference simulations is that complicated geometries like those with rough walls can be treated; see Stringano et al. (2006). The results-an enhanced heat flux consistent with $\mathrm{Nu}$ $\sim \mathrm{Ra}^{0.37}$ over the range $2 \times 10^{9} \leqslant \mathrm{Ra} \leqslant 2 \times 10^{11}$-are in reasonable agreement with the experimental results of Qiu et al. (2005), who found a power-law exponent of 0.35 in the range $10^{8} \leqslant \mathrm{Ra} \leqslant 10^{10}$, but in conflict with the earlier mentioned results by Du and Tong (2000), who found unchanged effective power-law exponents for the rough wall case, but a larger prefactor. Similarly, Verzicco (2003) found that by manipulating the velocity BC at the plates the viscous BL could be affected and also the absolute value of $\mathrm{Nu}$, but its power-law exponent was rather robust against such manipulations. Clearly, more research is necessary to clarify this matter. 
The numerical simulations of Shishkina and Wagner (2006, 2007, 2008) also are based on a finite volume scheme; they focus on the role of plumes and the flow organization in $\mathrm{RB}$ flow with $\mathrm{Pr}=0.7$ and $\mathrm{Pr}=5.4$ and $\mathrm{Ra}$ up to $2 \times 10^{9}$. For $\mathrm{Ra}=2 \times 10^{10}$ large-eddy simulations (LESs) were done. In this subgrid modeling the length scales in the dissipative and diffusive regime are underresolved.

Finally, when full spatial resolution of the turbulence field was abandoned even further, obviously much larger $\mathrm{Ra}$ could be achieved. An example for such a calculation is the so-called transient Reynolds averaged NavierStokes (RANS) method, which Kenjeres and Hanjalic (2002) applied to RB convection. They found $\mathrm{Nu}$ $\sim \mathrm{Ra}^{0.31}$ between $\mathrm{Ra}=10^{5}$ and $10^{15}$. For the last two $\mathrm{Ra}$ decades some reorganization of the plumes and a slightly enhanced Nusselt number were observed, but given the progressively decreasing spatial resolution of the numerical scheme at these high $\mathrm{Ra}$ the implications of this finding are presently unclear and a detailed discussion of RANS simulations and LES of RB flow is beyond the scope of this review.

Given the enormous CPU power needed to achieve large $\mathrm{Ra}$, one may wonder whether two-dimensional simulations would not be sufficient to reflect at least some aspects of the dynamics of the three-dimensional RB problem. This point has been analyzed by Schmalzl et al. (2002, 2004) whose conclusion is that for $\operatorname{Pr} \geqslant 1$ various properties observed in numerical $3 \mathrm{D}$ convection (and thus also in experiment) are indeed well reflected in 2D simulations. This in particular holds for the BL profiles and for the Nusselt number. Also Ahlers et al. (2008) and Sugiyama et al. (2007, 2009) employed 2D numerics to study the non-Oberbeck-Boussinesq deviations of the Nusselt number and of the bulk (central) temperature from that in the Oberbeck-Boussinesq cases; see Sec. VII.

\section{BOUNDARY LAYERS}

\section{A. Relevance of boundary layers and challenges}

Boundary layers describe the temperatures and flow fields in the vicinity of the plates and walls. They are characterized by their time-averaged profiles in the direction perpendicular to the respective solid boundary, i.e., in the $z$ direction off the bottom and top plates or in the $x$ direction off the sidewalls. As the top and bottom boundary layers contribute the main resistance for the heat transfer through the cell and thus dominantly determine the Nusselt number, they deserve special attention. Indeed, nearly all theories of $\mathrm{Nu}(\mathrm{Ra}, \mathrm{Pr})$ in $\mathrm{RB}$ convection are in essence boundary-layer theories: This holds for the now classical mixing-layer theory of Castaing et al. (1989), the turbulent-BL based scaling theory of Shraiman and Siggia (1990) and extensions thereof [e.g., Ching (1997)], the turbulent BL type theories of Dubrulle (2001, 2002) and Hölling and Herwig (2005, 2006), and the theory of Grossmann and Lohse (2000, 2001, 2002, 2004) which scalingwise builds heavily on the laminar, though time-dependent Prandtl-Blasius BL theory as elaborated in Sec. II.B. It is therefore of prime importance to directly study the BLs, to see whether the assumptions on which these theories are based are fulfilled at least to a reasonable approximation.

Unfortunately, directly studying the BLs in the large-Ra regime is equally challenging experimentally, numerically, and theoretically. Experimentally, the required spatial resolution is very difficult to achieve. Even for the $L \approx 6.5 \mathrm{~m}$ high Ilmenau RB barrel both the thermal and the kinetic $\mathrm{BL}$ thicknesses are only a few millimeters when, e.g., $\mathrm{Ra} \approx 10^{12}$; see du Puits, Resagk, and Thess (2007) and du Puits, Resagk, Tilgner, et al. (2007). In addition, both laser-Doppler velocimetry and hot-wire anemometry are difficult to employ in regions with large temperature fluctuations and a small meanflow velocity; both complications occur in the BLs. Numerically, large Rayleigh numbers are hard to reach, in particular with sufficient spatial resolution; see Sec. V. Even state-of-the-art DNS such as those of Verzicco and Sreenivasan (2008) have only a few grid points in the BLs. Theoretically, no generally accepted BL theory for flow over a strongly heated surface exists. Even in the truly laminar case an analytic theory for the two-way coupling of the temperature to the velocity field is missing (i.e., taking the temperature as an active scalar); see, e.g., Schlichting and Gersten (2000). And even if the two-way coupling is suppressed as done by Ching and Lo (2001) and Ching and Pang (2002), no exact analytical results exist for the thermal BL thickness and thus for the heat transfer through the shear flow over the heated plate.

The analysis of the BLs is further complicated by their extreme complexity, not only in time, where there is plume detachment (see Fig. 1), but also in space. Recent experimental studies such as those by Lui and $\mathrm{Xia}$ (1998), Wang and Xia (2003), Maystrenko et al. (2006), and Sun et al. (2008) and numerical studies such as those by Sugiyama et al. (2009) give increasing evidence that the BL thicknesses as well as the profiles of the mean quantities and of the fluctuations depend on the position along and above the plate(s), not only relative to the walls, but also relative to the fluctuating large convection roll (see Sec. IV). It is this main convection roll which creates the viscous BL because of the no-slip condition at the top and bottom plates.

The aim of this section is to give an overview of what has been found experimentally, theoretically, and numerically about the BL thicknesses.

\section{B. Thermal boundary layers}

The thermal boundary layer thickness can be defined in several ways. From an experimental point of view, it is easiest to time average the temperature profile at fixed lateral positions $(x, y)=*$ for various $z$ and to extract a thermal boundary layer thickness $\lambda_{\theta}(*)$ from the resulting local profile. From the theoretical viewpoint area averages on $x, y$ (in addition to the time averaging) are of main interest. Indeed, it is the thickness $\lambda_{\theta}$ of the area- 
averaged temperature profile in the $z$ direction which enters theory while comparison with experiment so far refers to locally measured BL thicknesses $\lambda_{\theta}(*)$. Note that strictly speaking the global thickness $\lambda_{\theta}$ is not equal to $\left\langle\lambda_{\theta}(*)\right\rangle_{A}$ in general; the reason is that the thickness is a (nonlinearly defined) property of a given profile. Then $\lambda_{\theta}$ in particular is a property of the area-averaged profile and not the average of properties $\lambda_{\theta}(*)$ of the respective local profiles. Along large plates the local BL width may well represent the area-averaged global one; but for RB samples with $\Gamma$ of order 1 one has to expect significant differences, even different scaling of the global and local thicknesses, since the area-averaged profiles comprise all inhomogeneities of the temperature and flow fields along the plates, including backflows, the regions between them, and the near wall ranges. These all will locally have quite different profiles. The more homogeneous the flow, the more similar the global width will be to the average of the local widths. Experimental analysis of these details is still a big challenge.

The (global) thermal BL thickness $\lambda_{\theta}$ can be defined in several ways. The most popular way is to define $\lambda_{\theta}$ through the slope of the area-averaged time-mean temperature profile at the plates: Take the tangent of the area-averaged mean temperature $\theta(z)$ at the plate. That distance between the plate and the vertical position where this tangent crosses the bulk (or center) temperature $T_{c}$ is then called $\lambda_{\theta}^{\mathrm{sl}}$. The center temperature $T_{c}$ is equal to the mean temperature $T_{m}=\left(T_{t}+T_{b}\right) / 2$ if the Oberbeck-Boussinesq approximation holds because of up-down symmetry. The very notion of $T_{c}$ already refers to an area-averaged profile.

Locally, i.e., for fixed $(x, y) \equiv *$, the slope of the timeaveraged profile, as well as the temperature for $z \rightarrow \infty$ and thus the local width $\lambda_{\theta}^{\mathrm{sl}}(*)$, will depend on the horizontal position $*$ where the local time-mean temperature profile is taken.

One of the first measurements of temperature and also velocity profiles in RB cells was by Tilgner et al. (1993), namely, in water $(\mathrm{Pr}=6.6)$ at fixed $\mathrm{Ra}=1.1 \times 10^{9}$ and at a fixed lateral position $*$. Belmonte et al. (1993) extended these measurements to the Ra range $5 \times 10^{5}$ $\leqslant \mathrm{Ra} \leqslant 10^{11}$ in compressed gas (air) at room temperature $(\operatorname{Pr}=0.7)$, but still at fixed lateral position. Lui and Xia (1998) measured the lateral dependence of $\lambda_{\theta}^{\mathrm{sl}}(*)$ on the positions $x$ (in the mean LSC direction) and $y$ (perpendicular to the preferred LSC direction) in a cylindrical water filled $\mathrm{RB}$ cell with $\Gamma=1$ in the regime $\mathrm{Ra}=2$ $\times 10^{8}-2 \times 10^{10}$. They reported variations of the $\mathrm{BL}$ width by nearly a factor of 2 , depending on the lateral location where the profile was measured. Wang and Xia (2003) found similar results for a cubic cell. Even the scaling exponent of $\lambda_{\theta}^{\mathrm{sl}}(*)$ with Ra depends on the position and varies between -0.35 and -0.28 . Lui and Xia (1998) found the thermal BL to be thinnest close to the center of the plates; there $2 \lambda_{\theta}^{\mathrm{sl}}(0,0) / L$ $=(0.23 \pm 0.02) \mathrm{Ra}^{-0.285 \pm 0.004}$ holds.

One cannot draw conclusions about the dependence of $\mathrm{Nu}$ on $\mathrm{Ra}$ from such measurements of local $\lambda_{\theta}^{\text {sl }}(*)$ at a particular lateral location. It is the length scale resulting from the laterally averaged BL temperature profile which is connected with the Nusselt number through the following exact relation:

$$
\mathrm{Nu}=\frac{\mid \partial_{z}\langle\theta(x, y, z=0 \text { or } z=L)\rangle_{A} \mid}{\Delta L^{-1}}=\frac{L}{2 \lambda_{\theta}^{\mathrm{sl}}}
$$

which follows from Eq. (5).

A different definition for a thermal boundary-layer thickness is the one employed by classical (laterally homogeneous) laminar BL theories (Prandtl, 1905; Blasius, 1908; see also Meksyn, 1961; Schlichting and Gersten, 2000; Cowley, 2001). This thickness, known as $\lambda_{\theta}^{99 \%}$, is defined as the vertical distance from the plate to the point where $99 \%$ of the temperature difference between plate and mean center temperature is achieved. For the bottom plate $\lambda_{\theta}^{99 \%}$ is the distance where the temperature reaches $T=T_{b}-0.99 \Delta_{b}$. This definition is in analogy to the common definition of the thickness $\delta$ of the kinetic BL, defined by the distance where, say, $99 \%$ of the maximum bulk velocity is achieved. The profile-based thickness $\lambda_{\theta}^{99 \%}$ can be calculated within the truly laminar Prandtl-Blasius BL theory, which implies lateral homogeneity and thus yields local and area-averaged widths that are the same. One obtains

$$
\frac{\lambda_{\theta}^{99 \%}}{L}=\frac{C(\operatorname{Pr})}{\operatorname{Re}^{1 / 2} \operatorname{Pr}^{1 / 3}},
$$

with a function $C(\operatorname{Pr})$ given by Meksyn (1961). For large Prandtl numbers $C(\operatorname{Pr})$ becomes constant, whereas for small $\operatorname{Pr}$ one finds $C(\operatorname{Pr}) \propto \operatorname{Pr}^{-1 / 6}$. We note that $\lambda_{\theta}^{99 \%}$ can display different scaling behavior than $\lambda_{\theta}^{\mathrm{sl}}$, as a function both of $\mathrm{Ra}$ and of $\mathrm{Pr}$, depending on the parameter-space regime. To our knowledge this issue has not yet been explored systematically, neither for $\lambda_{\theta}^{99 \%}$ nor for its local counterpart $\lambda_{\theta}^{99 \%}(*)$ which depends on the lateral position $*$ and refers to the local, only time-averaged, profiles.

A third way to define a thermal BL thickness, suggested by Belmonte et al. (1994), is to take the position of the maximal temperature fluctuations at the edge of the thermal BL. We call the respective BL thicknesses defined in this way $\lambda_{\theta}^{\sigma}$ and $\lambda_{\theta}^{\sigma}(*)$. du Puits, Resagk, Tilgner, et al. (2007) showed that thermal BL thicknesses defined in this way and measured in the barrel of Ilmenau using air at one atmosphere as the fluid behave differently from $\lambda_{\theta}^{\text {sl }}$. However, since BL thicknesses are determined largely by diffusive processes, it cannot be ruled out that a mixture like air (where both mass diffusion and heat diffusion play a role and where marginal stability depends on both concentration and temperature gradients) might in this respect behave differently from a pure fluid.

Profile measurements, and thus the determination of BL thicknesses, are obviously less difficult if the absolute length scale $L$ is larger. That is why the about $6.5 \mathrm{~m}$ high barrel of Ilmenau is particularly suited for these purposes. du Puits, Resagk, Tilgner, et al. (2007) mea- 

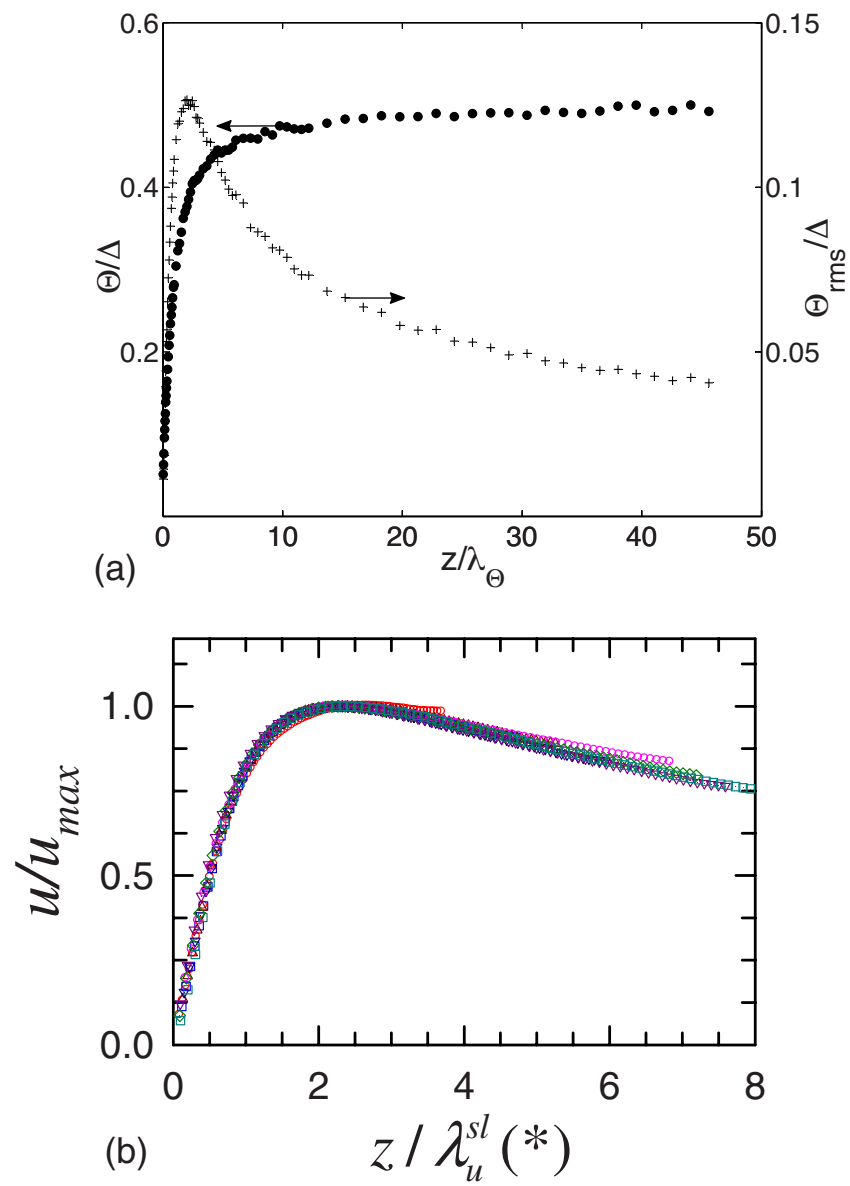

FIG. 9. (Color online) Temperature and velocity profiles. (a) Local, only time-averaged, temperature, and temperaturefluctuation profiles at $\mathrm{Ra}=7.7 \times 10^{11}$ and $\Gamma=1.13$ as functions of the height $z$ (normalized by $\lambda_{\theta}^{\mathrm{sl}}$ ), measured below the center of the top plate. The fluid is air at atmospheric pressure, the cell height is $6.3 \mathrm{~m}$. From du Puits, Resagk, Tilgner, et al., 2007. (b) Rescaled mean velocity profiles $U(z)$ above the center of the plate for $\mathrm{Ra}=1.25 \times 10^{9}$ (shortest data set) up to $\mathrm{Ra}=2.02$ $\times 10^{10}$ (longest data set) for RB flow in water. $u$ is normalized by $u_{\max }$ and $z$ by the kinetic BL thickness $\lambda_{u}^{s l}(*)$. From Sun et al., 2008.

sured local time-averaged temperature profiles up to $\mathrm{Ra}=7.7 \times 10^{11}$; for an example see Fig. 9(a). Outside a small linear regime near the plate they obtain a power law $\theta(z) \sim z^{\alpha}$ with $\alpha \approx 0.5$, only weakly dependent on Ra and $\Gamma$. For even larger $z$ the temperature saturated at its bulk value. The $1 / 2$ power law for intermediate heights supports neither the logarithmic scaling laws for turbulent BL theories nor the temperature profile for a oneway coupled temperature field (i.e., taking the temperature as a passive scalar) predicted by the (laminar) Prandtl-Blasius approach. However, the power law $\lambda_{\theta}^{\mathrm{sl}}(*) / L \propto \mathrm{Ra}^{-0.254}$ found by du Puits, Resagk, Tilgner, et al. (2007) below the center of the top plate is not inconsistent with what follows from the scalingwise laminar Prandtl-Blasius approach. Sun et al. (2008) obtained $\lambda_{\theta}^{\mathrm{sl}}(*) / L \propto \mathrm{Ra}^{-0.33 \pm 0.05}$ from temperature-profile measurements above the center of the plate in water with $\mathrm{Ra}$ up to $2 \times 10^{10}$.
Thermal BL thicknesses also have been determined from DNS. For a $\Gamma=1 \mathrm{RB}$ cell Verzicco and Camussi (1999) obtained for the area-averaged width $\lambda_{\theta}^{\text {sl }}$ $=3.1 \mathrm{Ra}^{-0.29}$ for $\operatorname{Pr}=0.7$ over the range $5 \times 10^{5} \leqslant \mathrm{Ra} \leqslant 2$ $\times 10^{7}$ and $\lambda_{\theta}^{\mathrm{sl}}=2.8 \mathrm{Ra}^{-0.25}$ for $\mathrm{Pr}=0.022$ in the regime 5 $\times 10^{4} \leqslant \mathrm{Ra} \leqslant 10^{6}$. When going towards the larger Ra regime $2 \times 10^{6} \leqslant \mathrm{Ra} \leqslant 2 \times 10^{11}$ Verzicco and Camussi (2003) found $\lambda_{\theta}^{\sigma} \sim \mathrm{Ra}^{-0.31}$ for $\operatorname{Pr}=0.7$ and $\Gamma=1 / 2$. For even larger Ra up to $2 \times 10^{14}$ Verzicco and Sreenivasan (2008) reported $\lambda_{\theta}^{\sigma} \sim \mathrm{Ra}^{-1 / 3}$.

The profiles of the rms temperature fluctuations are much less settled; see, e.g., Lui and Xia (1998), Fernandes and Adrian (2002), Wang and Xia (2003), du Puits, Resagk, Tilgner, et al. (2007), Sun et al. (2008). This issue will not be discussed in the present review.

As stated in Sec. II.B, thermal plumes can be viewed as detached pieces of the thermal BL. Since their discovery in RB convection by Zocchi et al. (1990) they have been studied intensively-experimentally, numerically, and theoretically - that a review of its own on this subject would be justified. A number of recent papers were devoted to them (Theerthan and Arakeri, 1998, 2000; Zhou and Xia, 2002; Breuer et al., 2004; Funfschilling and Ahlers, 2004; Haramina and Tilgner, 2004; Xi et al., 2004; Puthenveettil and Arakeri, 2005, 2008; Puthenveettil et al., 2005; Shishkina and Wagner, 2006, 2008; Zhou et al., 2007b) Their nature was discussed most recently by Funfschilling et al. (2008). Here we only make a few remarks. They seem to originate as onedimensional excitations of the marginally stable BL. As they are borne out of the BL, they become oriented by the LSC with their long axis in the direction of the flow, and then are swept by the LSC toward the sidewall. Along their way they separate from the BL and progress vertically near the wall, developing their famous mushroom top (Zocchi et al., 1990; Xi et al., 2004; Puthenveettil and Arakeri, 2005; Zhou et al., 2007b) in this process. Although they are often referred to as "sheetlike," implying an extension in two spatial dimensions, the term "linelike" would more appropriately describe their length scale of order $L$ in one dimension and of order the thermal boundary-layer thickness in the other two.

\section{Kinetic boundary layers}

The kinetic BL thicknesses $\lambda_{u}$ are determined from the velocity profiles or the velocity-fluctuation profiles. Also $\lambda_{u}$ can be defined in various ways, starting from either time- and area-averaged quantities or only locally time-averaged ones. An additional alternative here is to average the velocity vectors themselves or to take the rms velocities, componentwise or the full magnitude. Given the velocity profile of interest, its thickness may be defined (i) via the slope of the velocity profile in the $z$ direction (for the BL above or below the bottom or top plate) at a given position $*$ on the plate, known as $\lambda_{u}^{\text {sl }}(*)$, (ii) through the distance $\lambda_{u}^{m}(*)$ to the local maximal mean velocity, (iii) through the distance $\lambda_{u}^{\sigma}(*)$ to the maximal velocity rms fluctuations, etc. Again, all these 
quantities depend on the lateral position $*=(x, y)$, unless area averages are considered.

The first direct systematic measurements of velocity profiles in RB convection as a function of Ra were by Xin et al. (1996) and Xin and Xia (1997), using a novel light-scattering technique in a cylindrical cell. They found $\lambda_{u}^{\mathrm{sl}}(*) / L \sim \mathrm{Ra}^{-0.16}$ from the velocity profile above the center of the lower plate. At the sidewalls at half height as functions of $x$ instead of $z$ they measured $\lambda_{u}^{\text {sl }}(*) / L \sim \mathrm{Ra}^{-0.25}$. Qiu and Xia (1998a, 1998b) extended these measurements to convection in cubic cells, finding the same scaling exponents as in the cylindrical case. Using various organic liquids, Lam et al. (2002) could also explore the Pr dependence over the range $6 \leqslant \operatorname{Pr} \leqslant 1027$ and $2 \times 10^{8} \leqslant \mathrm{Ra} \leqslant 2 \times 10^{10}$, finding $\lambda_{u}^{\mathrm{sl}}(*) / L \sim \operatorname{Pr}^{0.24} \mathrm{Ra}^{-0.16}$ above the center plate position. The small $\mathrm{Ra}$ exponent of the effective power law is remarkable, as for a Prandtl-Blasius area-averaged BL profile one would expect an exponent of -0.25 , just as measured at the sidewalls, roughly corresponding to the Prandtl-Blasius scaling $\lambda_{u}^{\mathrm{sl}} / L \sim \mathrm{Re}^{-1 / 2}$ with (approximately) $\mathrm{Re} \sim \mathrm{Ra}^{1 / 2}$. We return to this point later. Note that these measurements were still done above a single position over the plate.

In 2003, particle image velocimetry (PIV) measurements revolutionarized the experimental analysis of the velocity field in thermal convection (Xia et al., 2003; Sun, Xi, and Xia, 2005; Sun, Xia, et al., 2005; Sun et al., 2006), including the analysis of the kinetic BLs (Sun et al., 2008). This allowed not only for the direct identification of various Reynolds numbers, cf. Sec. IV, but also for that of various kinetic BL thicknesses such as $\lambda_{u}^{\mathrm{sl}}(*)$, $\lambda_{u}^{m}(*)$, or $\lambda_{u}^{\sigma}(*)$, even at different positions $*$ in the cell.

For $10^{9} \leqslant \mathrm{Ra} \leqslant 10^{10}$ and $\operatorname{Pr}=4.3$ in a rectangular cell with $\Gamma=1$, Sun et al. (2008) obtained time-averaged velocity profiles in the center above the bottom plate. They found that if the vertical lengths are rescaled by $\lambda_{u}^{\text {sl }}(*)$ and the velocities by the maximal velocity, then the shape of the local velocity profile does not depend on $\mathrm{Ra}$; in this sense it is universal in that regime [see Fig. 9(b)]. Moreover, Sun et al. (2008) found $\lambda_{u}^{\mathrm{sl}}(*) / L \sim \lambda_{u}^{m}(*) / L \sim \mathrm{Ra}^{-0.27 \pm 0.01}$ [see Fig. 10(a)], in contrast to the much weaker Ra dependence reported earlier (Xin et al., 1996; Xin and Xia, 1997; Qiu and Xia, 1998a, 1998b). The origin of this discrepancy is not clear at this point. However, we note that the newly found scaling $\lambda_{u}^{\mathrm{sl}}(*) / L \sim \lambda_{u}^{m}(*) / L \sim \mathrm{Ra}^{-0.27 \pm 0.01}$ is consistent with the Prandtl-Blasius expectation. As a double check, Sun et al. (2008) plotted $\lambda_{u}^{\mathrm{sl}}(*) / L$ and $\lambda_{u}^{m}(*) / L$ against the independently determined Reynolds number, finding the Prandtl-Blasius scaling $\lambda_{u}^{\mathrm{sl}}(*) / L \sim \lambda_{u}^{m}(*) / L \sim \mathrm{Re}^{-0.50 \pm 0.03}$; see Fig. 10(b). In numerical simulations of RB flow for $\operatorname{Pr}=1$ and $\Gamma=1$ Breuer et al. (2004) found $\lambda_{u}^{\mathrm{sl}} / L$ $\sim \operatorname{Re}^{0.44 \pm 0.02}$, but a weaker $\operatorname{Re}$ dependence for $\lambda_{u}^{m} / L$. Numerical simulations by Verzicco and Camussi (1999) for the same $\Gamma=1$ and $\operatorname{Pr}=0.7$ gave an area-averaged profile thickness exponent consistent with the PrandtlBlasius BL theory, namely, $\lambda_{u}^{\text {sl }} / L=0.95 \mathrm{Ra}^{-0.23}$. For lower
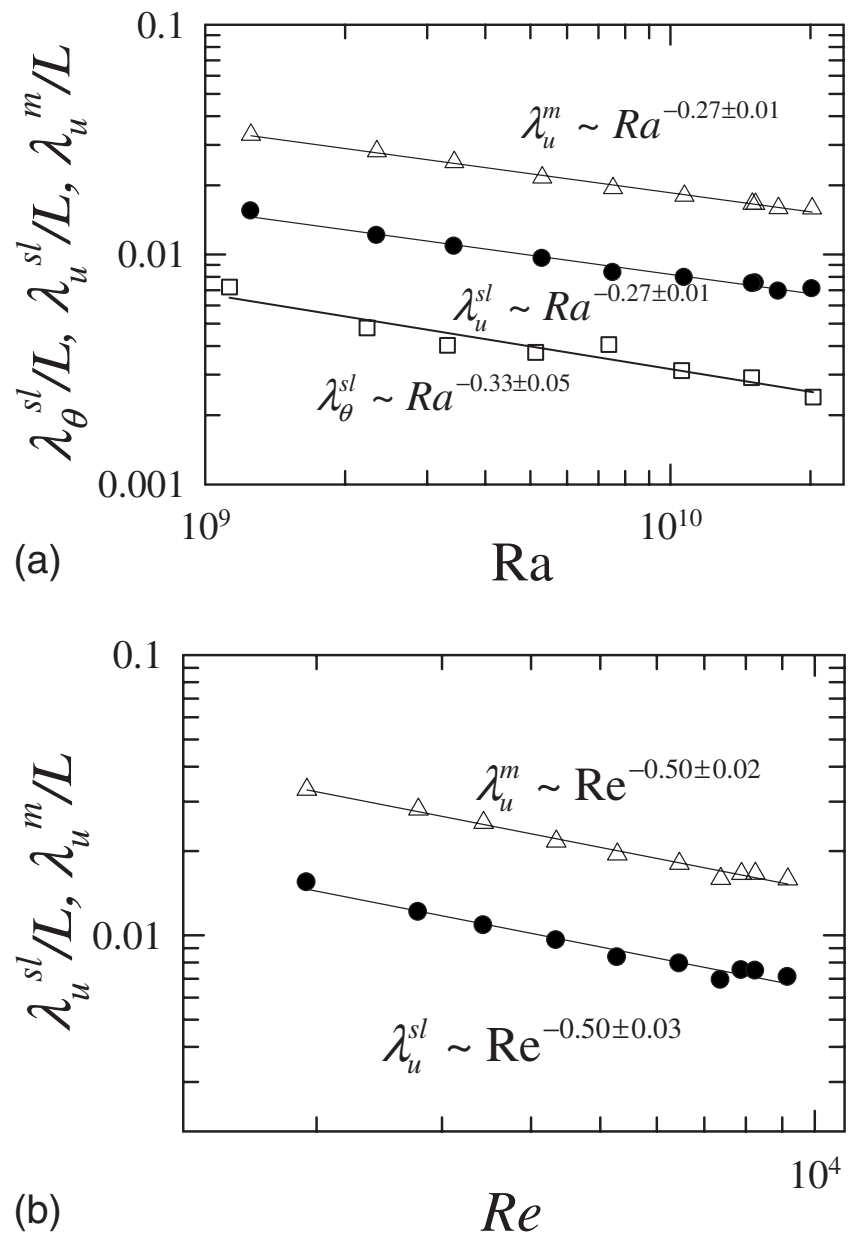

FIG. 10. Boundary layer thickness. Scaling of the kinetic BL thicknesses $\lambda_{u}^{\mathrm{sl}}(*)$ and $\lambda_{u}^{m}(*)$ as functions of (a) Ra and (b) Re, taken above the center of the lower plate. From Sun et al., 2008. In (a) the scaling of the thermal BL thickness $\lambda_{\theta}^{\mathrm{sl}}(*)$ is also shown. It has been deduced from the temperature profile above the center of the lower plate. The lines are power-law fits.

$\operatorname{Pr}=0.022$ Verzicco and Camussi (1999) obtained $\lambda_{u}^{\mathrm{sl}} / L$ $=0.1 \mathrm{Ra}^{-0.18}$.

The PIV BL study of Sun et al. (2008) also yielded the wall shear stress $\tau_{w}$, the viscous sublayer length scale $y_{w}=\nu(z=0) / u_{\tau}$, the skin-friction velocity $u_{\tau}=\left(\tau_{w} / \rho\right)^{1 / 2}$, and the skin-friction coefficient $c_{f}=\tau_{w} / \rho U_{\max }^{2}$. (These quantities have been measured above the plate center, but here for simplicity we suppress the $*=(x, y)$ dependence in the notation.) They found $\tau_{w} /\left(\rho \nu^{2} / L^{2}\right) \sim \mathrm{Re}^{1.55}$, $y_{w} / L \sim \operatorname{Re}^{-0.91}, u_{\tau} /(\nu / L) \sim \operatorname{Re}^{0.80}$, and $c_{f} \sim \operatorname{Re}^{-0.34}$. The respective Prandtl-Blasius scaling exponents for a laminar BL over a flat plate are $3 / 2,-1,3 / 4$, and $-1 / 2$. Apart from the last one all are in fair agreement with the measurements. It is hard to judge the importance of the discrepancy between -0.34 and -0.50 for the scaling of the skin-friction coefficient, in particular because the last three data points around $\operatorname{Re} \approx 10^{4}$ show a steeper dependence than -0.34; see Fig. 22(d) of Sun et al. (2008). Moreover, in the numerical simulations of Verzicco and Camussi (2003) $\left(\Gamma=1 / 2\right.$, Ra up to $\left.2 \times 10^{11}\right)$ the 
friction coefficient $c_{f}$ does show a Reynolds-number scaling exponent consistent with $-1 / 2$. (That work did not find any scaling for $\lambda_{u}^{\sigma}$ as function of Ra, presumably because of a reorganization of the flow in the $\Gamma=1 / 2$ cell with increasing Ra.) Also in the hitherto largest numerical RB simulations of Amati et al. (2005) $c_{f} \sim \mathrm{Re}^{-1 / 2}$ is found; see Fig. 6(b) of that paper. Next, also the experimental analysis of Chavanne et al. (2001) found a $-1 / 2$ scaling of a surrogate of the friction factor with Re up to $\mathrm{Ra}=10^{11}$. However, at $\mathrm{Ra}=10^{11}$, corresponding to $\mathrm{Re}$ $=8 \times 10^{4}$, the data of Chavanne et al. (2001) give evidence for a transition towards a weaker dependence. Verzicco and Camussi (2003) found the same transition in this friction factor surrogate, but not in the actual friction factor $c_{f}$.

Finally, we mention the interesting numerical study by Yu et al. (2007), who analyzed the heat transfer and boundary-layer thicknesses in laminar and steady convection with $\mathrm{Ra}$ up to $10^{8}$, finding basically the same Ra-scaling laws for $\mathrm{Nu}, \lambda_{u}$, and $\lambda_{\theta}$ as in classical RB convection. Their conclusion is that turbulence does not play a decisive role for the heat transfer.

In summary, although some of the various velocity BL profiles differ from the idealized Prandtl-Blasius truly laminar profile due to the permanently ongoing plume emission, the scaling of the kinetic BL thickness with $\mathrm{Ra}, \mathrm{Re}$, and $\mathrm{Pr}$ is consistent with the laminar PrandtlBlasius theory at least up to $\mathrm{Ra}=10^{11}$, but presumably also beyond. There is no indication of any transition towards a different kinetic BL thickness scaling even at the largest $\mathrm{Ra}$ realized up to now. Moreover, for $\operatorname{Pr} \gtrsim 1$ the thermal $\mathrm{BL}$ is nested in the kinetic one, whereas for $\operatorname{Pr} \leq 1$ it is the other way around.

The scaling of the rms velocity fluctuations [see, e.g., du Puits, Resagk, and Thess (2007) and Sun et al. (2008) for recent work on this issue] will not be discussed in this review.

\section{NON-OBERBECK-BOUSSINESQ EFFECTS}

The problem of $\mathrm{RB}$ convection is commonly analyzed within the so-called Oberbeck-Boussinesq (OB) approximation (Oberbeck, 1879; Boussinesq, 1903), in which the fluid properties are assumed to be temperature independent, apart from the density for which the linear temperature dependence equation (1) is assumed. Under normal conditions, i.e., "small" temperature differences $\Delta$ between the bottom and top plates, this approximation is rather good. However, in order to achieve ever larger values of Ra for given $L$ and fluid properties $\beta, c_{p}, \kappa$, and $\nu$, the temperature difference $\Delta$ between top and bottom plates quite frequently was increased to such an extent that the OB approximation had to be expected to fail. Non-Oberbeck-Boussinesq (NOB) effects, i.e., deviations of various properties including $\mathrm{Nu}$ and the center temperature $T_{c}$ from the $\mathrm{OB}$ case, then had to be expected at the largest $\mathrm{Ra}$ in several experiments (Castaing et al., 1989; Chavanne et al., 1997; Ashkenazi and Steinberg, 1999; Niemela et al., 2000a;
Niemela and Sreenivasan, 2003a, 2006b). Particularly problematic were measurements in the vicinity of liquidgas critical points, where Re tends to become exceptionally large but where NOB effects are to be expected at relatively small $\Delta$. To be able to interpret these high-Ra data-in particular, with respect to the question of whether there is an intrinsic large-Ra transition in the flow-it is therefore important to understand the physical nature, the signatures, and the size of the NOB effects. In addition to this practical consideration, there is much interesting physics to be learned from their study.

NOB effects in high-Ra convection were measured and analyzed first using helium gas by $\mathrm{Wu}$ and Libchaber (1991), and then using water and glycerol by Zhang et al. (1997). They confirmed experimentally that the temperature dependence of the fluid properties leads to a symmetry breaking between the top and the bottom of the sample. The temperature drops $\Delta_{b}$ and $\Delta_{t}$ across the bottom and top boundary layers become different, $\Delta_{b} \neq \Delta_{t}$, and so do the thicknesses of the thermal BLs, $\lambda_{\theta, b}^{\mathrm{sl}} \neq \lambda_{\theta, t}^{\mathrm{sl}}$. Both phenomena are associated with a corresponding shift of the temperature $T_{c}=T_{b}-\Delta_{b}=T_{t}$ $+\Delta_{t}$ in the center (bulk) of the cell away from the arithmetic mean temperature $T_{m}=\left(T_{b}+T_{t}\right) / 2$ of the bottom and top plate temperatures $T_{b}$ and $T_{t}$. Moreover, one would expect NOB observables to include deviations of $\mathrm{Nu}$ and $\mathrm{Re}$ from their $\mathrm{OB}$ values if $\Delta$ becomes large. Surprisingly, Wu and Libchaber (1991) and Zhang et al. (1997) found that $\mathrm{Nu}$ is remarkably insensitive to NOB effects. To our knowledge, NOB effects on Re have yet to be observed experimentally.

In order to assess the validity of the $\mathrm{OB}$ approximation, Niemela and Sreenivasan (2003a) suggested that the following three measures are basically equivalent: Busse's weighted sum of fractional deviations $\left(X_{t}\right.$ $\left.-X_{b}\right) / X_{m}$ for the relevant material properties $X$, the ratio $\chi=\Delta_{b} / \Delta_{t}$ [introduced by Wu and Libchaber (1991)] of the temperature drops across the bottom and top BLs, and the relative change of the density $\beta \Delta$. The last criterion is the simplest, and Niemela and Sreenivasan (2003a) suggested on empirical grounds that OB conditions can reasonably be expected to prevail when $\beta \Delta$ is less than about $0.1-0.2$. We show that the situation is more complicated and that there is a plethora of different NOB effects.

NOB effects for RB convection in water were measured and analyzed systematically by Ahlers, Brown, Fontenele Araujo, et al. (2006). Of the relevant fluid properties, the kinematic viscosity $\nu$ had the largest temperature dependence in this case. They compared $\mathrm{Nu}$ for three samples with different $L$, but the same Ra, all with $\Gamma=1$. The same $\mathrm{Ra}$ was realized in different ways by keeping $L^{3} \Delta$ constant while $L$ and $\Delta$ were changed. On the one hand, NOB effects were negligible in a cell with large $L$ and sufficiently small $\Delta$. On the other, a cell with relatively small $L$ but larger $\Delta$ up to $40 \mathrm{~K}$ could have strong NOB effects. For the largest $\Delta$ the deviation of the center temperature $T_{c}$ from the mean temperature $T_{m}$ was only about $1.8 \mathrm{~K}$ [see Fig. 11(c)], corresponding 


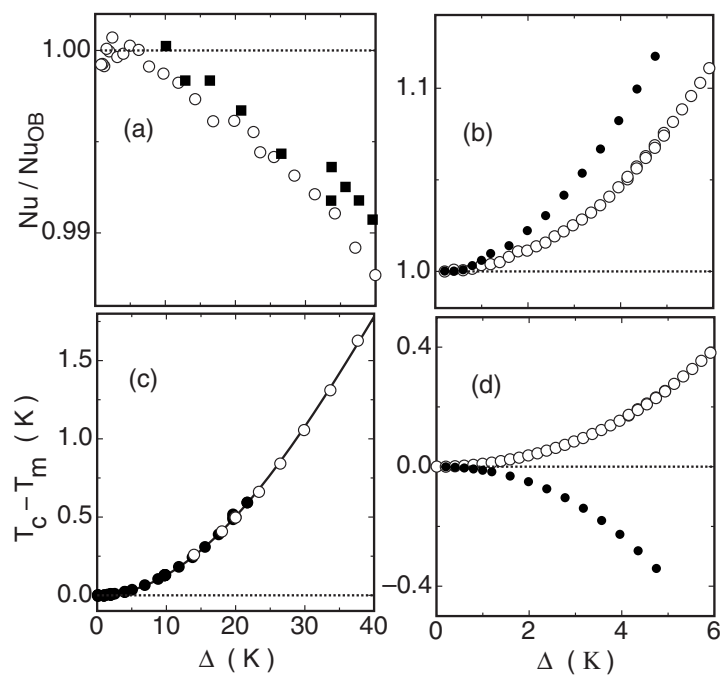

FIG. 11. Deviations from the Oberbeck-Boussinesq approximation. (a), (b) The ratio $\mathrm{Nu} / \mathrm{Nu}_{\mathrm{OB}}$ as a function of $\Delta$ for fixed $T_{m}$. (c), (d) $T_{c}-T_{m}$ as a function of $\Delta$, also fixed $T_{m}$. (a), (c) Water at $T_{m}=40.0{ }^{\circ} \mathrm{C}, \Gamma=1.0$. Solid circles, $L=49.7 \mathrm{~cm}$. Open circles, $L=24.8 \mathrm{~cm}$. Solid squares, $L=9.2 \mathrm{~cm}$. (b), (d) Ethane at a pressure of 55.17 bars for $\Gamma=0.5$. Open symbols, $T_{m}$ $=35.0^{\circ} \mathrm{C}$ below the critical isochore temperature $T_{\phi}$ $=38.06{ }^{\circ} \mathrm{C}$ at this pressure. Solid symbols, $T_{m}=41.0^{\circ} \mathrm{C}$, above $T_{\phi}$.

to $\chi \simeq 0.85$. Brown, Fontenele Araujo, Ahlers, et al. (2006) calculated the increase of $T_{c}$ theoretically by developing an extended Prandtl-Blasius BL theory, which takes the $T$ dependence of $\nu$ and $\kappa$ into account.

Surprisingly, for these water measurements the NOB effects on $\mathrm{Nu}$ were small, see Fig. 11(a): less than $1.4 \%$ even for $\Delta=40 \mathrm{~K}$. To account for this finding, Ahlers, Brown, Fontenele Araujo, et al. (2006) derived an extension of the exact relation (32) for the Nusselt number to the NOB case, proving the generally valid exact relation

$$
\frac{\mathrm{Nu}}{\mathrm{Nu}_{\mathrm{OB}}}=\frac{2 \lambda_{\theta, \mathrm{OB}}^{\mathrm{sl}}}{\lambda_{\theta, b}^{\mathrm{sl}}+\lambda_{\theta, t}^{\mathrm{sl}}} \frac{\kappa_{b} \Delta_{b}+\kappa_{t} \Delta_{t}}{\kappa_{m} \Delta} \equiv F_{\lambda} F_{\Delta} .
$$

In each of the two factors $F_{\lambda}$ and $F_{\Delta}$ the symmetrybreaking different bottom and top BL properties enter additively and thus tend to compensate each other, leading to a cancellation of the linear contributions to their temperature dependences. Thus the remaining NOB effects on $\mathrm{Nu}$ originate only from the quadratic and higher-order variations of the material properties with temperature.

With the help of Eq. (34) Ahlers, Brown, Fontenele Araujo, et al. (2006) could identify the origins of the NOB corrections for water: For this fluid it is mainly the temperature dependence of the thermal diffusivity $\kappa$ that is responsible for the $\mathrm{NOB}$ correction of $\mathrm{Nu}$, while the NOB correction of the center temperature $T_{c}$ has its main origin in the temperature dependence of the kinematic viscosity $\nu$. Instead in glycerol, which displays an extreme dependence of $\nu$ on the temperature, the NOB corrections for $T_{c}$ are much larger, both in experiment
(Zhang et al., 1997) and in the two-dimensional numerical simulations of Sugiyama et al. (2007).

Theoretical calculations of $T_{c}$ from the extended Prandtl-Blasius BL theory of Ahlers, Brown, Fontenele Araujo, et al. (2006) for various waterlike fluids with hypothetical temperature dependences of the material parameters were in good agreement with the corresponding numerical results of Sugiyama et al. (2007, 2009). Ahlers et al. (2007) applied the extended Prandtl-Blasius theory of Ahlers, Brown, Fontenele Araujo, et al. (2006) to NOB effects in gases, finding good agreement for $T_{c}$ with experiments using pressurized ethane gas, in which the material properties strongly depend on temperature. For this case a decrease of $T_{c}$ relative to $T_{m}$ and an increase of $\mathrm{Nu}$ in comparison to the $\mathrm{OB}$ case was found. Note that both effects are in the opposite directions as compared to the NOB effects in water or glycerol.

In experiments by Ahlers et al. (2008) the measurements using ethane were extended to the region near the critical point; see Figs. 11(b) and 11(d). On first sight the results for $T_{c}$ seem surprising. NOB effects in liquidlike ethane ( $\rho>\rho_{c}$ where $\rho_{c}$ is the critical density) caused an increase of $T_{c}$ relative to $T_{m}$, whereas in gaslike ethane $\left(\rho<\rho_{c}\right)$ NOB effects made $T_{c}$ smaller than $T_{m}$. The physical reason for this qualitative difference was found in the opposite sign of the bottom-top asymmetry of the buoyancy strength due to the opposite temperature dependence of $\beta(T)$ for the two cases. In the liquidlike case the buoyancy, proportional to $\beta(T)=\beta\left(T_{m}\right)+(T$ $\left.-T_{m}\right) \beta^{\prime}\left(T_{m}\right)$, is larger at the bottom and smaller at the top, supporting the uprising warmer bottom plumes more than the down-coming colder top plumes. This brings predominantly hotter material into the bulk, leading to $T_{c}>T_{m}$. For gaslike ethane the buoyancy is larger at the cooler top, favoring down-going cold over uprising warm plumes. This in turn brings more cooler material into the bulk, resulting in $T_{c}<T_{m}$. It is the sign of the slope $\beta^{\prime}$ of $\beta$ at $T_{m}$ that is the relevant quantity for this type of NOB correction.

Of course, the extended Prandtl-Blasius BL theory fails when the thermal expansion coefficient $\beta$, which is disregarded in that theory by construction as it treats the temperature as a passive scalar, shows an extreme temperature dependence such as close to a criticial point. The above explanation in terms of the strong temperature dependence of $\beta$, which leads to broken buoyancy symmetry, as an additional cause of NOB effects on $T_{c}$ was verified numerically by Ahlers et al. (2008).

Even though the two cases $\rho<\rho_{c}$ and $\rho>\rho_{c}$ discussed above had $T_{c}$ displaced away from $T_{m}$ in opposite directions, they both yielded an enhancement of $\mathrm{Nu}$ above the $\mathrm{OB}$ value, i.e., in the same direction despite opposite deviations of $T_{c}$ from $T_{m}$. This insensitiveness to the sign of $T_{c}-T_{m}$ can be understood from Eq. (34). Physically, the BLs act as two thermal resistances in series, since Nusselt number deviations only depend on the sum of both, and it does not matter much which of them is reduced and which one is enhanced.

The flow organization due to NOB effects in water 

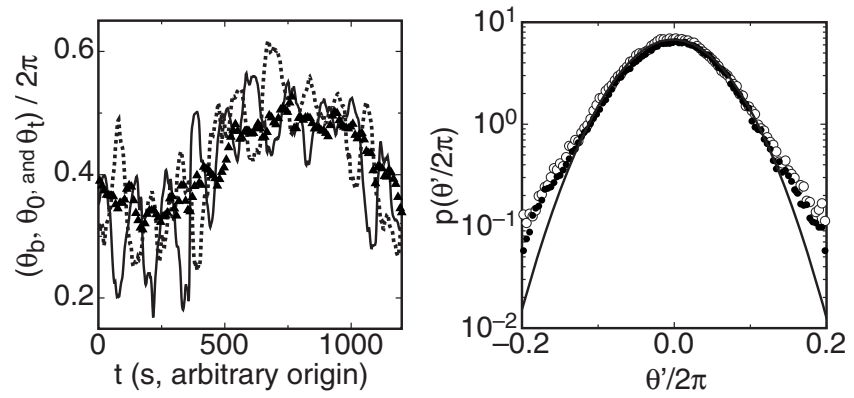

FIG. 12. Behavior of LSC. Left: The LSC orientation as a function of time at $z=-L / 4\left(\theta_{b}\right.$, dotted line $), z=0\left(\theta_{0}\right.$, triangles), and $z=L / 4\left(\theta_{t}\right.$, solid line $)$. Right: The probability distribution $p\left(\theta^{\prime} / 2 \pi\right)$ as a function of $\theta^{\prime} / 2 \pi$. Here $\theta^{\prime}=\theta_{t}-\theta_{0}$ (open circles) and $\theta^{\prime}=\theta_{b}-\theta_{0}$ (solid circles). Solid line, a Gaussian distribution. Adapted from Funfschilling et al., 2008.

and other liquids was studied numerically by Sugiyama et al. (2009), again with 2D RB simulations. It was confirmed that buoyancy, in particular, the temperature dependence of the thermal expansion coefficient $\beta$, is the main origin of the NOB effects on the centerroll Reynolds number, which roughly behaves like $\mathrm{Re} / \operatorname{Re}_{O B} \approx\left[\beta\left(T_{c}\right) / \beta\left(T_{m}\right)\right]^{1 / 2}$. Reynolds-number measurements using water (Ahlers, Brown, Fontenele Araujo, et al., 2006) were still unable to resolve NOB effects within their resolution of $1 \%$ or $2 \%$ even though $\Delta$ was as large as $38 \mathrm{~K}$.

\section{GLOBAL WIND DYNAMICS}

\section{A. Experiment}

For cylindrical samples with $\Gamma=1$ the LSC circulation plane breaks the rotational invariance of the cell. This leads to interesting dynamics which includes oscillations of the circulation plane (Heslot et al., 1987; Castaing et al., 1989; Ciliberto et al., 1996; Takeshita et al., 1996; Cioni et al., 1997; Qiu et al., 2000, 2004; van Doorn et al., 2000; Niemela et al., 2001; Qiu and Tong, 2001a, 2001b, 2002; Sreenivasan et al., 2002; Xi et al., 2006; Resagk et al., 2006; Xi and Xia, 2007). These are caused by a torsional mode in which the orientation of the upper half of the LSC undergoes azimuthal oscillations (Funfschilling and Ahlers, 2004; Resagk et al., 2006) that are out of phase with those of the lower half (Funfschilling and Ahlers, 2004; Funfschilling et al., 2008). This is shown in the left part of Fig. 12, which shows the LSC azimuthal orientations (characterized by the angle) $\theta_{b}$ at the vertical position $z=-L / 4$, the azimuthal orientation $\theta_{0}$ at $z$ $=0$, and the azimuthal orientation $\theta_{t}$ at $z=+L / 4$ (the origin of the $z$ axis is at the cell center). These orientations were determined by measuring the azimuthal temperature variation of the side wall at the three vertical positions. Casual inspection shows that $\theta_{t}$ and $\theta_{b}$ oscillate, out of phase with each other and perhaps with random amplitudes, about $\theta_{0}$. Quantitative analysis using correlation functions confirms this qualitative result.

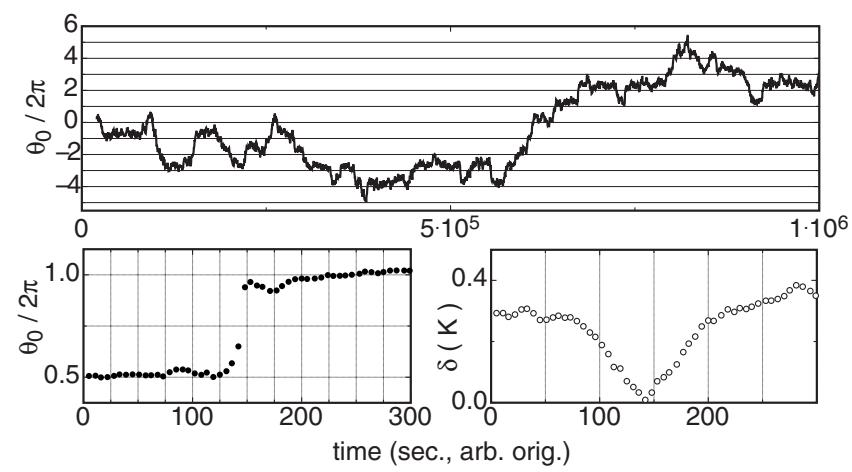

FIG. 13. Time dependence of LSC orientation. Top: A time series of $\theta_{0}(t)$ spanning about 11 days. Adapted from Brown and Ahlers, 2006b. Bottom left: The LSC orientation $\theta_{0}(t)$. Bottom right: The LSC azimuthal temperature amplitude $\delta(t)$ at the sidewall, during a cessation. Adapted from Brown, Nikolaenko, et al., 2005.

Important insight into the origin of the twisting mode is gained from the probability distribution functions $p\left(\theta_{b, t}^{\prime}\right)$ shown in the right part of Fig. 12. Here $p\left(\theta_{b, t}^{\prime}\right)$ is the probability of the azimuthal displacement $\theta_{b, t}^{\prime} \equiv \theta_{b, t}$ $-\theta_{0}$, relative to $\theta_{0}$, of $\theta_{b}$ or $\theta_{t}$. If the mode had its origin in a Hopf bifurcation, then one would expect it to have a characteristic finite amplitude $A$ which would lead to two peaks of $p\left(\theta_{b, t}^{\prime}\right)$ at or near $\theta^{\prime}= \pm A$. In contrast to this, the experimental result for $p\left(\theta_{b, t}^{\prime}\right)$ is a nearGaussian distribution with only one maximum centered at $\theta_{t, b}^{\prime}=0$. Such a distribution is characteristic of a damped oscillator driven by a broad-band noise source [see, for instance, Gitterman (2005)]. This driving is attributed to the action of the small scale turbulent fluctuations on the large scale flow.

The twisting mode does not influence the LSC orientation $\theta_{0}$ at $z=0$ because there it has a node. However, the LSC breaks the rotational symmetry of a cylindrical sample and its circulation plane must somehow choose an azimuthal orientation. This orientation has been found to undergo spontaneous diffusive meandering (Sun, Xi, and Xia, 2005; Brown and Ahlers, 2006a, 2006b; Xi et al., 2006) in the sense that its mean square azimuthal displacement is proportional to the elapsed time. This diffusion is shown in the top of Fig. 13 by a long time series of $\theta_{0}(t)$. It is also attributed to the action of the small scale fluctuations on the large scale flow. Measurements of its diffusivity have yielded results for the intensity of the fluctuating force (Brown and Ahlers, 2006a, 2006b).

Recently Xi et al. (2009) and Zhou, Xi, et al. (2009) discovered a "sloshing" mode in a $\Gamma=1$ cylindrical sample that occurs in addition to the torsional mode and that was missed by the analysis method employed by Funfschilling and Ahlers (2004) and Funfschilling et al. (2008). This mode consists of an in-phase horizontal displacement of the entire LSC. A theoretical explanation of the origin of both modes was given recently by Brown and Ahlers (2009). The sloshing mode is responsible for the oscillations seen in some of the local temperature 
measurements that had been attributed by some to periodic plume emission from the thermal boundary layers.

The LSC also undergoes re-orientations both by azimuthal rotations (Cioni et al., 1997; Brown and Ahlers, 2006b) and by cessations in which the LSC slows to a stop and restarts in a random new orientation (Brown, Nikolaenko, et al., 2005; Brown and Ahlers, 2006b; Xi and Xia, 2007). A cessation is shown in the lower part of Fig. 13. The left part reveals a sudden change in orientation. The right one shows that the temperature amplitude $\delta$, and thus presumably the LSC itself, vanishes or comes close to zero at the time of the cessation. The angular change $\Delta \theta_{c}$ during a cessation has a nearly uniform probability distribution, indicating that once the flow stops it re-organizes itself in a random new orientation. The angular change $\Delta \theta_{r}$ of rotations has a powerlaw distribution, with small changes more likely then large ones. The time interval $\tau_{1}$ between successive events for either cessations or rotations is Poisson distributed, showing that successive events are uncorrelated.

On longer time scales, the Earth's Coriolis force (at a latitude of $34^{\circ}$ in the Northern Hemisphere) was found to cause a net clockwise (as seen from above) rotation of the LSC orientation on average once every 3 days, and to align the LSC in a preferred orientation close to West. The measured probability distribution $p\left(\theta_{0}\right)$ agreed quantitatively with a model calculation of the Coriolisforce interaction that involved no adjustable parameters (Brown and Ahlers, 2006a). The net clockwise rotation was also consistent with this model.

The LSC in other geometries and aspect ratios adds to the richness of this phenomenon but is beyond the scope of this review. We mention briefly that DNS revealed two counter-rotating near-circular rolls, stacked vertically one above the other (Verzicco and Camussi, 2003) as the dominating feature of the LSC in cylindrical samples with $\Gamma \leqq 0.5$. Recent experimental evidence provided by Xi and Xia (2008b) for $\Gamma=0.5$ and 0.33 indicates random temporal successions of one-roll and two-roll states, with the two-roll state becoming more prevalent as $\Gamma$ is decreased. Xi and Xia (2008a) found a strong aspect ratio dependence when comparing the azimutal motion, reorientation, cessation, and reversal of the large scale circulation in cylindrical RB samples with $\Gamma$ $=2.3,1$, and 0.5. In samples of square or rectangular cross section the LSC is locked in a predominant orientation along a diagonal (Daya and Ecke, 2001). This is now understood in terms of the pressure gradients that arise when the rotational invariance of a cylindrical sample is broken (Brown and Ahlers, 2008a). Zhou et al. (2007a) studied temperature and velocity oscillations in a rectangular cell finding that the temperature oscillations scale differently with $\mathrm{Ra}$ than the velocity oscillations, which are affected by the cell geometry. Of great interest, but largely unexplored, is the flow structure that will be found in large- $\Gamma$ systems where numerous convection rolls potentially can coexist next to each other. Recent papers addressing this issue numerically include that by Hartlep et al. (2005). Recent experimental work going in this direction includes the measurements by Niemela and Sreenivasan (2006a).

\section{B. Models}

Stochastic models of flow reversal have been proposed by Sreenivasan et al. (2002) and Benzi (2005). They treated diffusion of the LSC strength in a potential well, but there was no physical motivation for the shape of the potential that was used and the model parameters were chosen phenomenologically. They also did not address the azimuthal dynamics of the LSC. Two other models describe the LSC with deterministic ordinary differential equations that have chaotic solutions (Fontenele Araujo et al., 2005; Resagk et al., 2006). They were derived by retaining some relevant aspects of the Navier-Stokes equations and making various approximations. The model of Fontenele Araujo et al. (2005) is based on assumptions about the lifetimes $\tau_{p}$ of plumes. It is estimated that plumes with a sufficiently large $\tau_{p}$ will be carried over by the LSC to the far side where their buoyancy tends to act against the prevailing flow. This physical process can lead to cessations. Since this model does not contain an azimuthal mode, it cannot describe the rich azimuthal dynamics of the physical system.

Recently a model consisting of two stochastic ordinary differential equations, one each for the circulation strength $U$ and the azimuthal circulation-plane orientation $\theta_{0}$, was developed by Brown and Ahlers (2007a, 2008b). Starting with the Navier-Stokes (NS) equation, it was argued that $U$ is driven by the buoyancy term and hindered by the dissipation in the viscous boundary layers near the walls. A phenomenological stochastic driving term representing the interaction between the small scale turbulent fluctuations and the large scale circulation is added to the deterministic model. For $\theta_{0}$ the only driving is the turbulent fluctuations, but the component of the nonlinear term in the NS equation that describes the rotational inertia of the LSC provides damping and couples the two equations. Assuming that the experimentally accessible azimuthal temperature amplitude $\delta$ is proportional to $U$ near the side wall, the model becomes

$$
\dot{\delta}=\frac{\delta}{\tau_{\delta}}-\frac{\delta^{3 / 2}}{\tau_{\delta} \sqrt{\delta_{0}}}+f_{\delta}(t), \quad \ddot{\theta}_{0}=-\frac{\dot{\theta}_{0} \delta}{\tau_{\theta} \delta_{0}}+f_{\dot{\theta}}(t)
$$

with the coefficients

$$
\delta_{0} \equiv \frac{18 \pi \Delta \sigma \mathrm{Re}^{3 / 2}}{R}, \quad \tau_{\delta} \equiv \frac{L^{2}}{18 \nu \mathrm{Re}^{1 / 2}}, \quad \tau_{\theta} \equiv \frac{L^{2}}{2 \nu \mathrm{Re}} .
$$

The intensities of the stochastic forces $f_{\delta}(t)$ and $f_{\dot{\theta}}(t)$ are obtained from model-independent measurements of the diffusivities of $\delta$ and $\dot{\theta}_{0}$. 
The potential corresponding to the $\dot{\delta}$ equation has a $\delta^{5 / 2}$ rather than the usual quartic nonlinearity. However, this does not change its qualitative structure, which has an unstable fixed point at $\delta=0$ and a stable one at $\delta$ $=\delta_{0}$. The driving will cause diffusion in the vicinity of the stable fixed point, with occasional excursions to $\delta=0$ corresponding to cessations. During a cessation (when $\delta$ is small) the damping term on the right hand side of the $\ddot{\theta}_{0}$ equation, and thus the angular momentum of the LSC, become small, and it becomes easy for the stochastic driving to cause relatively large angular changes $\Delta \theta_{c}$. The model agrees well with many experimental results for the LSC dynamics (Brown and Ahlers, 2008b), including a time interval between cessations of 1-2 days, a near-uniform distribution $p\left(\Delta \theta_{c}\right)$ for cessations, and the dependence of $\delta_{0}$ on $\mathrm{Re}$ and $\mathrm{Ra}$ expressed by $\left(\delta_{0} / \Delta\right)(\mathrm{Ra} / \operatorname{Pr}) \propto \mathrm{Re}^{3 / 2}$. There is, however, a significant disagreement between the model and the measured tail of the probability distribution $p(\delta)$ at small $\delta$, for $\delta$ $\lesssim \delta_{0} / 2$. It has been suggested (Brown and Ahlers, 2008b) that this is caused by the neglect of thermal conduction across the thermal BLs which is expected to become important when $\delta$ is small.

Recently the above model was extended by Brown and Ahlers (2008a) by including various perturbations that break the rotational invariance of the sample. These include (i) the effect of Earth's Coriolis force, (ii) an elliptic rather than circular horizontal cross section of the sample, (iii) a tilt of the sample axis relative to gravity, and (iv) a small horizontal temperature gradient at the top or bottom plate. It turns out that (i) and (ii) only influence the $\ddot{\theta}_{0}$ equation and not the $\dot{\delta}$ equation. Perturbations of this type, although they introduce a preferred orientation of the LSC, leave the Reynolds number and the frequency of cessations largely unchanged. However, perturbations like (iii) and (iv) which affect $\dot{\delta}$ influence Re and suppress cessations. A tilt or elliptic eccentricity of sufficient magnitude creates a new oscillatory mode of the LSC which is different from the torsional oscillation in that the phase of the oscillation is uniform along the height of the sample. For the tilted sample this mode was found in recent experiments (Brown and Ahlers, 2008a).

\section{ISSUES FOR FUTURE RESEARCH}

As shown in this review, since Siggia's article (1994) tremendous progress in the understanding of the turbulent Rayleigh-Bénard system has been achieved by experiment, theory, and numerical simulation. However, it has also become clear that our understanding is far from complete. In the following we summarize what we consider as major issues for future research.

Presumably the most important challenge is to clarify whether and, if so, where in parameter space the ultimate state of convection exists. Estimates suggest that (for Pr near 1) a transition to such an ultimate state should occur at Rayleigh numbers around $10^{13}-10^{14}$. Be- yond that transition the Nusselt number should increase more rapidly with $\mathrm{Ra}$ than below it. Though the Grenoble experiments suggest such a transition near $\mathrm{Ra}=10^{11}$, neither the Oregon-Trieste experiments nor numerical simulations do so. The reason for the discrepancy is unresolved at present. It is important to clarify this issue in order to allow extrapolations of the heat transfer to the very large Ra regime of geophysical and astrophysical interest. Perhaps related to this issue is whether a coherent LSC continues to exist at very large $\mathrm{Ra}$, or whether it is totally overwhelmed by fluctuations. Several experimental efforts are planned to try to answer these questions.

Rather than focusing on global quantities such as the heat flux, which may be difficult to control in the largescale setups necessary in an ultimate regime, a promising complementary strategy may be to focus on a detailed analysis of the top and bottom BLs, whose structure should reflect such a transition. Here the challenge is the opposite: Extremely small structures must be spatially resolved. Even for an about 7-m-high sample (such as the barrel of Ilmenau) the thermal boundary layer is only about $1 \mathrm{~mm}$ thick when $\mathrm{Ra}=10^{14}$. Helpful strategies may be to try to trigger the transition in the $\mathrm{BL}$, by controlled roughness or even by moving parts.

Also the three-dimensional dynamics of the large scale convection roll will need further attention and analysis. As shown in Sec. VIII, it is rather rich, including torsional modes, rotation, cessation, and sloshing. Here the key question is: What features of the LSC can be described through a deterministic model somehow based on the Oberbeck-Boussinesq dynamics or some force balance and what features need stochastic elements for a description? Routes for further research on this subject will include experiments and numerical simulations with modified geometries as, e.g., cylindrical samples with a horizontal axis.

Another issue for future research is the exploration of the aspect-ratio dependence of the flow. Though the $\Gamma$ dependence of $\mathrm{Nu}$ is found experimentally to be weak, a full understanding of the RB system requires further theoretical efforts also in that direction. For the characterization of the corresponding flow organizations detailed PIV measurements could play a crucial role. These will also shed light on the Ra and Pr dependences of the various Reynolds numbers that one can define, and their connections.

From the theoretical viewpoint, the GL theory has provided a good guideline for the understanding of $\mathrm{Nu}(\mathrm{Ra}, \mathrm{Pr})$ and $\mathrm{Re}(\mathrm{Ra}, \mathrm{Pr})$ and even allowed various predictions, but the theory has its limitations

- It builds on the Prandtl-Blasius BL theory for the temperature as a passive scalar. The buoyancy term is skipped by construction in the Prandtl BL equations, but it is of high importance for thermally driven flow, leading to plume detachment from the thermal BLs. This detachment mechanism as a timedependent BL separation process needs further study and analysis of its parameter dependences. The 
GL theory only represents the global effect of the plumes and the resulting self-organizing buoyancydriven flow, namely, the large scale wind.

- The three-dimensional (torsional) dynamics of the large scale wind is not taken into consideration by the GL theory (or any other model).

- Experiments suggest that $\mathrm{Nu}$ and $\mathrm{Re}$ are not as intimately related as the relations (21) and (22) of the GL theory suggest. For instance, by tilting the system $\mathrm{Re}$ can be enhanced considerably whereas $\mathrm{Nu}$ changes very little.

- Figure 4 implies that there are more sudden transitions between the various regimes in reality than within the GL theory.

- Finally, in the $\mathrm{Nu} \propto \mathrm{Ra}^{1 / 3}$ regime the experimentally found coexistence of a measured Reynolds number $\propto \mathrm{Ra}^{1 / 2}$, achieved in Santa Barbara, and the GL prediction $\mathrm{Re} \propto \mathrm{Ra}^{4 / 9}$ for the global wind has to be clarified and resolved.

More theoretical work is necessary to improve on the GL theory. This unfortunately may only be possible by sacrificing its conceptual appeal and simplicity.

An important further extension of the present theoretical understanding of $\mathrm{RB}$ convection, but of course also of the numerical simulations and the experiments, is the extension towards rotating $\mathrm{RB}$ convection. With rotation, the $(\mathrm{Ra}, \mathrm{Pr})$ parameter space is extended to ( $\mathrm{Ra}, \mathrm{Pr}, \mathrm{Ro}$ ), where the Rossby number Ro is defined as $\operatorname{Ro}=(2 \Omega)^{-1} \sqrt{\beta g \Delta / L}$, i.e., as the ratio of the time scale given by the rotation rate $\Omega$ and the one given by buoyancy. The obvious questions to address are: What is the dependence of the Nusselt number on the control parameters $\mathrm{Ra}, \mathrm{Pr}$, and $\mathrm{Ro}$, i.e., what is $\mathrm{Nu}(\mathrm{Ra}, \mathrm{Pr}, \mathrm{Ro})$ ? $^{8}$ And how do the large scale convection roll and the Reynolds number react to the rotation, i.e., what is $\operatorname{Re}(\mathrm{Ra}, \mathrm{Pr}, \mathrm{Ro})$ ? When will the large scale convection roll break down? How are the top-bottom, and sidewall kinetic and thermal BLs modified through the rotation? Only a small fraction of the parameter space $(\mathrm{Ra}, \mathrm{Pr}, \mathrm{Ro})$ has hitherto been explored. Given that rotating turbulence is known for its counterintuitive features (e.g., the Taylor-Proudman effect), we expect many surprising results ahead of us. We do not want to give an extensive literature review on rotating RB here, but good starting points are Greenspan (1990) or the classical article by Rossby (1969) himself.

Future developments undoubtedly will also include the extension of the current state of the art of RB convection to complex fluids, to fluids containing bubbles or suspensions of particles, to fluids undergoing phase transitions, and so on. But these topics are beyond the scope of the present review.

Fifteen years after Siggia's article on RB convection (Siggia, 1994), and inspite of the huge progress achieved

\footnotetext{
${ }^{8}$ For a very recent reference on this issue we refer to Zhong et al. (2009), and references therein.
}

during this time, we close with a similar statement as he did: The current rate of experimental and numerical advances will again soon antiquate this summary.

\section{ACKNOWLEDGMENTS}

We thank all our co-workers and colleagues for their contributions to our understanding of this great problem, for the many stimulating discussions we had the privilege to enjoy over the years, and for their many valuable comments on this manuscript. The work of G.A. was supported in part by U.S. National Science Foundation Grant No. DMR07-02111 and by the Alexander von Humboldt Stiftung. D.L. acknowledges FOM for the continuous support.

\section{REFERENCES}

Acrivos, A., 2008, private communication.

Ahlers, G., 1974, "Low temperature studies of the RayleighBénard instability and turbulence," Phys. Rev. Lett. 33, 11851188.

Ahlers, G., 1975, in Fluctuations, Instabilities and Phase Transitions, edited by T. Riste (Plenum, New York), pp. 181-193. Ahlers, G., 2000, "Effect of sidewall conductance on heattransport measurements for turbulent Rayleigh-Bénard convection," Phys. Rev. E 63, 015303(R).

Ahlers, G., E. Brown, F. Fontenele Araujo, D. Funfschilling, S. Grossmann, and D. Lohse, 2006, "Non-Oberbeck-Boussinesq effects in strongly turbulent Rayleigh-Bénard convection," J. Fluid Mech. 569, 409-445.

Ahlers, G., E. Brown, and A. Nikolaenko, 2006, "The search for slow transients, and the effect of imperfect vertical alignment, in turbulent Rayleigh-Bénard convection," J. Fluid Mech. 557, 347-367.

Ahlers, G., E. Calzavarini, F. Fontenele Araujo, D. Funfschilling, S. Grossmann, D. Lohse, and K. Sugiyama, 2008, "NonOberbeck-Boussinesq effects in turbulent thermal convection in ethane close to the critical point," Phys. Rev. E 77, 046302. Ahlers, G., F. Fontenele Araujo, D. Funfschilling, S. Grossmann, and D. Lohse, 2007, "Non-Oberbeck-Boussinesq effects in strongly turbulent Rayleigh-Bénard convection," Phys. Rev. Lett. 98, 054501.

Ahlers, G., and X. Xu, 2001, "Prandtl-number dependence of heat transport in turbulent Rayleigh-Bénard convection," Phys. Rev. Lett. 86, 3320-3323.

Amati, G., K. Koal, F. Massaioli, K. R. Sreenivasan, and R. Verzicco, 2005, "Turbulent thermal convection at high Rayleigh numbers for a constant-Prandtl-number fluid under Boussinesq conditions," Phys. Fluids 17, 121701.

Ashkenazi, S., and V. Steinberg, 1999, "High Rayleigh number turbulent convection in a gas near the gas-liquid critical point,” Phys. Rev. Lett. 83, 3641-3644.

Balachandar, S., M. R. Maxey, and L. Sirovich, 1989, "Numerical simulation of high Rayleigh number convection," J. Sci. Comput. 4, 219-236.

Behringer, R. P., 1985, "Rayleigh-Bénard convection and turbulence in liquid-helium," Rev. Mod. Phys. 57, 657-687.

Belmonte, A., A. Tilgner, and A. Libchaber, 1993, "Boundary layer length scales in thermal turbulence," Phys. Rev. Lett. 70, 4067-4070.

Belmonte, A., A. Tilgner, and A. Libchaber, 1994, "Tempera- 
ture and velocity boundary layers in turbulent convection," Phys. Rev. E 50, 269-279.

Benzi, R., 2005, "Flow reversal in a simple dynamical model of turbulence," Phys. Rev. Lett. 95, 024502.

Benzi, R., F. Toschi, and R. Tripiccione, 1998, "On the heat transfer in the Rayleigh-Bénard system," J. Stat. Phys. 93, 901-918.

Benzi, R., R. Tripiccione, F. Massaioli, S. Succi, and S. Ciliberto, 1994, "Scaling behavior of the velocity and temperature correlation-functions in 3D convective turbulence," Europhys. Lett. 25, 341.

Blasius, H., 1908, "Grenzschichten in Flüssigkeiten mit kleiner Reibung," Z. Math. Phys. 56, 1-37.

Bodenschatz, E., W. Pesch, and G. Ahlers, 2000, "Recent developments in Rayleigh-Bénard convection," Annu. Rev. Fluid Mech. 32, 709-778.

Bolgiano, R., 1959, "Turbulent spectra in a stably stratified atmosphere," J. Geophys. Res. 64, 2226.

Boussinesq, J., 1903, Theorie Analytique de la Chaleur (Gauthier-Villars, Paris), Vol. 2.

Brent, A. D., V. R. Voller, and K. J. Reid, 1988, "Enthalpyporosity technique for modeling convection-diffusion phase change-Application to the melting of a pure metal," Numer. Heat Transfer 13, 297-318.

Breuer, M., S. Wessling, J. Schmalzl, and U. Hansen, 2004, "Effect of inertia in Rayleigh-Bénard convection," Phys. Rev. E 69, 026302.

Brown, E., and G. Ahlers, 2006a, "Effect of the Earth's Coriolis force on turbulent Rayleigh-Bénard convection in the laboratory," Phys. Fluids 18, 125108.

Brown, E., and G. Ahlers, 2006b, "Rotations and cessations of the large-scale circulation in turbulent Rayleigh-Bénard convection," J. Fluid Mech. 568, 351-386.

Brown, E., and G. Ahlers, 2007a, "Large-scale circulation model for turbulent Rayleigh-Bénard convection," Phys. Rev. Lett. 98, 134501.

Brown, E., and G. Ahlers, 2007b, "Temperature gradients, and search for non-Boussinesq effects, in the interior of turbulent Rayleigh-Bénard convection,” Europhys. Lett. 80, 14001.

Brown, E., and G. Ahlers, 2008a, "A dynamical model of the large-scale circulation in turbulent Rayleigh-Bénard convection: azimuthal asymmetries," Phys. Fluids 20, 105105.

Brown, E., and G. Ahlers, 2008b, "A model of diffusion in a potential well for the dynamics of the large-scale circulation in turbulent Rayleigh-Bénard convection," Phys. Fluids 20, 075101.

Brown, E., and G. Ahlers, 2009, "The origin of oscillations of the large-scale circulation of turbulent Rayleigh-Bénard convection," e-print arXiv:0902.4487.

Brown, E., D. Funfschilling, and G. Ahlers, 2007, “Anomalous Reynolds-number scaling in turbulent Rayleigh-Bénard convection," J. Stat. Mech.: Theory Exp. 2007, P10005.

Brown, E., D. Funfschilling, A. Nikolaenko, and G. Ahlers, 2005, "Heat transport by turbulent Rayleigh-Bénard convection: Effect of finite top- and bottom conductivity," Phys. Fluids 17, 075108.

Brown, E., A. Nikolaenko, and G. Ahlers, 2005, "Reorientation of the large-scale circulation in turbulent RayleighBénard convection," Phys. Rev. Lett. 95, 084503.

Busse, F. H., 1969, "On Howard's upper bound for heat transport in turbulent convection," J. Fluid Mech. 37, 457-477.

Busse, F. H., 2003, "The sequence-of-bifurcations approach towards understanding turbulent fluid flow," Surv. Geophys. 24,
269-288

Calzavarini, E., C. R. Doering, J. D. Gibbon, D. Lohse, A. Tanabe, and F. Toschi, 2006, "Exponentially growing solutions of homogeneous Rayleigh-Bénard flow," Phys. Rev. E 73, 035301(R).

Calzavarini, E., D. Lohse, F. Toschi, and R. Tripiccione, 2005, "Rayleigh and Prandtl number scaling in the bulk of Rayleigh-Bénard turbulence," Phys. Fluids 17, 055107.

Calzavarini, E., F. Toschi, and R. Tripiccione, 2002, "Evidences of Bolgiano-Obhukhov scaling in three-dimensional Rayleigh-Bénard convection,” Phys. Rev. E 66, 016304.

Camussi, R., and R. Verzicco, 1999, "Convective turbulence in mercury: Scaling laws and spectra," Phys. Fluids 10, 516-527. Cardin, P., and P. Olson, 1994, "Chaotic thermal convection in a rapidly rotating spherical shell: Consequences for flow in the outer core," Phys. Earth Planet. Inter. 82, 235-259.

Castaing, B., G. Gunaratne, F. Heslot, L. Kadanoff, A. Libchaber, S. Thomae, X. Z. Wu, S. Zaleski, and G. Zanetti, 1989, "Scaling of hard thermal turbulence in RayleighBénard convection," J. Fluid Mech. 204, 1-30.

Cattaneo, F., T. Emonet, and N. Weiss, 2003, "On the interaction between convection and magnetic fields," Astrophys. J. 588, 1183-1198.

Chandrasekhar, S., 1981, Hydrodynamic and Hydromagnetic Stability (Dover, New York).

Chaumat, S., B. Castaing, and F. Chilla, 2002, in Advances in Turbulence IX, edited by I. P. Castro, P. E. Hancock, and T. G. Thomas (International Center for Numerical Methods in Engineering, CIMNE, Barcelona).

Chavanne, X., F. Chilla, B. Castaing, B. Hebral, B. Chabaud, and J. Chaussy, 1997, "Observation of the ultimate regime in Rayleigh-Bénard convection,” Phys. Rev. Lett. 79, 3648-3651. Chavanne, X., F. Chillá, B. Chabaud, B. Castaing, J. Chaussy, and B. Hébral, 1996, "High Rayleigh number convection with gaseous helium at low temperature," J. Low Temp. Phys. 104, 109-129.

Chavanne, X., F. Chilla, B. Chabaud, B. Castaing, and B. Hebral, 2001, "Turbulent Rayleigh-Bénard convection in gaseous and liquid He," Phys. Fluids 13, 1300-1320.

Chillà, F., M. Rastello, S. Chaumat, and B. Castaing, 2004a, "Long relaxation times and tilt sensitivity in Rayleigh-Bénard turbulence," Eur. Phys. J. B 40, 223-227.

Chillà, F., M. Rastello, S. Chaumat, and B. Castaing, 2004b, "Ultimate regime in Rayleigh-Bénard convection: The role of plates," Phys. Fluids 16, 2452-2456.

Ching, E. S. C., 1997, "Heat flux and shear rate in turbulent convection," Phys. Rev. E 55, 1189-1192.

Ching, E. S. C., H. Guo, X. D. Shang, P. Tong, and K.-Q. Xia, 2004, "Extraction of plumes in turbulent thermal convection," Phys. Rev. Lett. 93, 124501.

Ching, E. S. C., and K. F. Lo, 2001, "Heat transport by fluid flows with prescribed velocity fields," Phys. Rev. E 64, 046302.

Ching, E. S. C., and K. M. Pang, 2002, "Dependence of heat transport on the strength and shear rate of circulating flows," Eur. Phys. J. B 27, 559-564.

Ching, E. S. C., and W. S. Tam, 2006, “Aspect-ratio dependence of heat transport by turbulent Rayleigh-Bénard convection," J. Turbul. 7, 1-10.

Cholemari, M. R., and J. H. Arakeri, 2005, "Experiments and a model of turbulent exchange flow in a vertical pipe," Int. J. Heat Mass Transfer 48, 4467-4473.

Cholemari, M. R., and J. H. Arakeri, 2009, “Axially homoge- 
neous, zero-mean flow buoyancy driven turbulence in a vertical pipe," J. Fluid Mech. 621, 69.

Christie, S. L., and J. A. Domaradzki, 1992, "Numerical evidence for nonuniversality of the soft/hard turbulence classification for thermal convection," Phys. Fluids A 5, 412-421.

Chu, T. Y., and R. J. Goldstein, 1973, "Turbulent convection in a horizontal layer of water," J. Fluid Mech. 60, 141-159.

Ciliberto, S., S. Cioni, and C. Laroche, 1996, "Large-scale flow properties of turbulent thermal convection," Phys. Rev. E 54, R5901-R5904.

Ciliberto, S., and C. Laroche, 1999, "Random roughness of boundary increases the turbulent convection scaling exponent," Phys. Rev. Lett. 82, 3998-4001.

Cioni, S., S. Ciliberto, and J. Sommeria, 1995, “Temperature structure functions in turbulent convection at low Prandtl number," Europhys. Lett. 32, 413-418.

Cioni, S., S. Ciliberto, and J. Sommeria, 1996, "Experimental study of high Rayleigh-number convection in mercury and water," Dyn. Atmos. Oceans 24, 117-127.

Cioni, S., S. Ciliberto, and J. Sommeria, 1997, "Strongly turbulent Rayleigh-Bénard convection in mercury: Comparison with results at moderate Prandtl number," J. Fluid Mech. 335, 111-140.

Constantin, P., and C. Doering, 1999, "Infinite Prandtl number convection,” J. Stat. Phys. 94, 159-172.

Cowley, S. J., 2001, in Mechanics for a New Millenium, ICTAM2000 (Chicago) Proceedings, edited by H. Aref and J. W. Philips (Kluwer Academic, Dordrecht), pp. 389-411.

Davis, A. H., 1922a, "Natural convective cooling of wires," Philos. Mag. 43, 329-339.

Davis, A. H., 1922b, "Natural convective in cooling in fluids," Philos. Mag. 44, 920-940.

Daya, Z. A., and R. E. Ecke, 2001, "Does turbulent convection feel the shape of the container?," Phys. Rev. Lett. 87, 184501.

DeLuca, E. E., J. Werne, R. Rosner, and F. Cattaneo, 1990, "Numerical simulations of soft and hard turbulencePreliminary results for two-dimensional convection," Phys. Rev. Lett. 64, 2370-2373.

Doering, C., and P. Constantin, 1996, "Variational bounds on energy dissipation in incompressible flows: III. Convection," Phys. Rev. E 53, 5957-5981.

Doering, C. R., and P. Constantin, 1992, "Energy-dissipation in shear driven turbulence,” Phys. Rev. Lett. 69, 1648-1651.

Doering, C. R., F. Otto, and M. G. Reznikoff, 2006, "Bounds on vertical heat transport for inifinite-Prandtl-number Rayleigh-Bénard convection," J. Fluid Mech. 560, 229-241.

Drazin, P., and W. H. Reid, 1981, Hydrodynamic Stability (Cambridge University Press, Cambridge, England).

Du, Y. B., and P. Tong, 2000, "Turbulent thermal convection in a cell with ordered rough boundaries," J. Fluid Mech. 407, 57-84.

Du, Y. B., and P. Tong, 2001, "Temperature fluctuations in a convection cell with rough upper and lower surfaces," Phys. Rev. E 63, 046303.

Dubrulle, B., 2001, "Momentum transport and torque scaling in Taylor-Couette flow from an analogy with turbulent convection," Eur. Phys. J. B 21, 295.

Dubrulle, B., 2002, "Scaling in large Prandtl number turbulent thermal convection," Eur. Phys. J. B 28, 361-367.

du Puits, R., C. Resagk, and A. Thess, 2007, "Mean velocity profile in confined turbulent convection," Phys. Rev. Lett. 99, 234504.

du Puits, R., C. Resagk, A. Tilgner, F. H. Busse, and A. Thess,
2007, "Structure of thermal boundary layers in turbulent Rayleigh-Bénard convection,” J. Fluid Mech. 572, 231-254.

Eckhardt, B., S. Grossmann, and D. Lohse, 2000, "Scaling of global momentum transport in Taylor-Couette and pipe flow," Eur. Phys. J. B 18, 541-544.

Eckhardt, B., S. Grossmann, and D. Lohse, 2007a, "Fluxes and energy dissipation in thermal convection and shear flows," Europhys. Lett. 78, 24001.

Eckhardt, B., S. Grossmann, and D. Lohse, 2007b, "Torque scaling in turbulent Taylor-Couette flow between independently rotating cylinders," J. Fluid Mech. 581, 221-250.

Fernandes, R. L. J., and R. J. Adrian, 2002, "Scaling of the velocity and temperature fluctuations in turbulent thermal convection," Exp. Therm. Fluid Sci. 26, 355-360.

Fleischer, A. S., and R. J. Goldstein, 2002, "High-Rayleighnumber convection of pressurized gases in a horizontal enclosure,” J. Fluid Mech. 469, 1-12.

Fontenele Araujo, F., S. Grossmann, and D. Lohse, 2005, "Wind reversals in turbulent Rayleigh-Bénard convection," Phys. Rev. Lett. 95, 084502.

Frisch, U., 1995, Turbulence (Cambridge University Press, Cambridge, England).

Funfschilling, D., and G. Ahlers, 2004, "Plume motion and large scale circulation in a cylindrical Rayleigh-Bénard cell," Phys. Rev. Lett. 92, 194502.

Funfschilling, D., E. Brown, and G. Ahlers, 2008, "Torsional oscillations of the large-scale circulation in turbulent Rayleigh-Bénard convection,” J. Fluid Mech. 607, 119-139.

Funfschilling, D., E. Brown, A. Nikolaenko, and G. Ahlers, 2005, "Heat transport by turbulent Rayleigh-Bénard convection in cylindrical cells with aspect ratio one and larger," J. Fluid Mech. 536, 145-154.

Getling, A. V., 1998, Rayleigh-Bénard Convection: Structures and Dynamics (World Scientific, Singapore).

Gibert, M., H. Pabiou, F. Chilla, and B. Castaing, 2006, "HighRayleigh-number convection in a vertical channel," Phys. Rev. Lett. 96, 084501

Gitterman, M., 2005, The Noisy Oscillator, The First Hundred Years, From Einstein Until Now (World Scientific, Singapore). Glatzmaier, G. A., and P. H. Roberts, 1995, “A 3-dimensional self-consistent computer simulation of a geomagnetic field reversal," Nature (London) 377, 203-209.

Glazier, J. A., T. Segawa, A. Naert, and M. Sano, 1999, "Evidence against ultrahard thermal turbulence at very high Rayleigh numbers," Nature (London) 398, 307-310.

Greenspan, H. P., 1990, The Theory of Rotating Flows (Breukelen Press, Brookline, MA).

Grossmann, S., and D. Lohse, 1993, "Characteristic scales in Rayleigh-Bénard convection," Phys. Lett. A 173, 58.

Grossmann, S., and D. Lohse, 2000, "Scaling in thermal convection: A unifying view," J. Fluid Mech. 407, 27-56.

Grossmann, S., and D. Lohse, 2001, "Thermal convection for large Prandtl number," Phys. Rev. Lett. 86, 3316-3319.

Grossmann, S., and D. Lohse, 2002, "Prandtl and Rayleigh number dependence of the Reynolds number in turbulent thermal convection," Phys. Rev. E 66, 016305.

Grossmann, S., and D. Lohse, 2003, "On geometry effects in Rayleigh-Bénard convection,” J. Fluid Mech. 486, 105-114. Grossmann, S., and D. Lohse, 2004, "Fluctuations in turbulent Rayleigh-Bénard convection: The role of plumes," Phys. Fluids 16, 4462-4472.

Grossmann, S., and D. Lohse, 2008, in "Thermal convection in small Prandtl number liquids: strong but ineffective," Let's 
Face Chaos through Nonlinear Dynamics: Proceedings of the 7th International Summer School and Conference, edited by M. Robnik and V. G. Romanovski, AIP Conf. Proc. No. 1076 (AIP, Melville, NY), pp. 68-75.

Grötzbach, G., 1982, "Direct numerical simulation of laminar and turbulent Bénard convection," J. Fluid Mech. 119, 27-53. Grötzbach, G., 1983, "Spatial resolution for direct numerical simulations of Rayleigh-Bénard convection," J. Comput. Phys. 49, 241-264.

Haramina, T., and A. Tilgner, 2004, "Coherent structures in boundary layers of Rayleigh-Bénard convection," Phys. Rev. E 69, 056306.

Hartlep, T., A. Tilgner, and F. H. Busse, 2003, "Large scale structures in Rayleigh-Bénard convection at high Rayleigh numbers," Phys. Rev. Lett. 91, 064501.

Hartlep, T., A. Tilgner, and F. H. Busse, 2005, "Transition to turbulent convection in a fluid layer heated from below at moderate aspect ratio," J. Fluid Mech. 544, 309-322.

Hartmann, D. L., L. A. Moy, and Q. Fu, 2001, "Tropical convection and the energy balance at the top of the atmosphere," J. Clim. 14, 4495-4511.

Heslot, F., B. Castaing, and A. Libchaber, 1987, "Transition to turbulence in helium gas," Phys. Rev. A 36, 5870-5873.

Hirschfelder, J. O., C. F. Curtiss, and R. B. Bird, 1964, Molecular Theory of Gases and Liquids (Wiley, New York).

Hölling, M., and H. Herwig, 2005, "Asymptotic analysis of the near-wall region of turbulent natural convection flows," $\mathrm{J}$. Fluid Mech. 541, 383-397.

Hölling, M., and H. Herwig, 2006, "Asymptotic analysis of heat transfer in turbulent Rayleigh-Bénard convection," Int. J. Heat Mass Transfer 49, 1129-1136.

Horanyi, S., L. Krebs, and U. Müller, 1999, "Turbulent Rayleigh-Bénard convection in low Prandtl number fluids," Int. J. Heat Mass Transfer 42, 3983-4003.

Howard, L. N., 1963, "Heat transport by turbulent convection," J. Fluid Mech. 17, 405-432.

Howard, L. N., 1972, "Bounds on flow quantities," Annu. Rev. Fluid Mech. 4, 473-494.

Hunt, G. R., and P. F. Linden, 1999, "The fluid mechanics of natural ventilation-Displacement ventilation by buoyancydriven flows assisted by wind," Build. Environ. 34, 707-720.

Johnston, H., and C. R. Doering, 2007, "Rayleigh-Bénard convection with imposed heat flux," Chaos 17, 041103.

Johnston, H., and C. R. Doering, 2009, "Comparison of turbulent thermal convection between conditions of constant temperature and constant flux," Phys. Rev. Lett. 102, 064501.

Kadanoff, L. P., 2001, "Turbulent heat flow: Structures and scaling," Phys. Today 54 (8), 34-39.

Kenjeres, S., and K. Hanjalic, 2002, "Numerical insight into flow structure in ultraturbulent thermal convection," Phys. Rev. E 66, 036307.

Kerr, R., 1996, "Rayleigh number scaling in numerical convection," J. Fluid Mech. 310, 139-179.

Kerr, R., and J. R. Herring, 2000, "Prandtl number dependence of Nusselt number in direct numerical simulations," $\mathrm{J}$. Fluid Mech. 419, 325-344.

Kraichnan, R. H., 1962, "Turbulent thermal convection at arbritrary Prandtl number," Phys. Fluids 5, 1374-1389.

Krishnamurti, R., and L. N. Howard, 1981, "Large scale flow generation in turbulent convection," Proc. Natl. Acad. Sci. U.S.A. 78, 1981-1985.

Kunnen, R. P. J., H. J. H. Clercx, B. J. Geurts, L. A. Bokhoven, R. A. D. Akkermans, and R. Verzicco, 2008, "A numerical and experimental investigation of structure function scaling in turbulent Rayleigh-Bénard convection," Phys. Rev. E 77, 016302.

Lam, S., X. D. Shang, S. Q. Zhou, and K.-Q. Xia, 2002, "Prandtl-number dependence of the viscous boundary layer and the Reynolds-number in Rayleigh-Bénard convection," Phys. Rev. E 65, 066306.

Landau, L. D., and E. M. Lifshitz, 1987, Fluid Mechanics (Pergamon, Oxford).

Lohse, D., and F. Toschi, 2003, "The ultimate state of thermal convection," Phys. Rev. Lett. 90, 034502.

Lohse, D., and K.-Q. Xia, 2010, "Small-scale properties of turbulent Rayleigh-Bénard convection," Ann. Rev. Fluid Mech. (to be published).

Lui, S. L., and K.-Q. Xia, 1998, "Spatial structure of the thermal boundary layer in turbulent convection," Phys. Rev. E 57, 5494-5503.

Malkus, M. V. R., 1954, "The heat transport and spectrum of thermal turbulence," Proc. R. Soc. London, Ser. A 225, 196212.

Marshall, J., and F. Schott, 1999, "Open-ocean convection: Observations, theory, and models," Rev. Geophys. 37, 1-64.

Maystrenko, A., C. Resagk, and A. Thess, 2006, "Structure of the thermal boundary layer for turbulent Rayleigh-Bénard convection of air in a long rectangular enclosure," Phys. Rev. E 75, 066303.

McKenzie, D. P., J. M. Roberts, and N. O. Weiss, 1974, "Convection in the Earth's mantle: towards a numerical simulation," J. Fluid Mech. 62, 465-538.

Meksyn, D., 1961, New Methods in Laminar Boundary Layer Theory (Pergamon, Oxford).

Monin, A. S., and A. M. Yaglom, 1975, Statistical Fluid Mechanics (MIT, Cambridge, MA).

Naert, A., T. Segawa, and M. Sano, 1997, "High-Reynoldsnumber thermal turbulence in mercury," Phys. Rev. E 56, R1302-R1305.

Niemela, J., L. Skrbek, K. R. Sreenivasan, and R. Donnelly, 2000a, "Turbulent convection at very high Rayleigh numbers," Nature (London) 404, 837-840.

Niemela, J., L. Skrbek, K. R. Sreenivasan, and R. J. Donnelly, 2001, "The wind in confined thermal turbulence," J. Fluid Mech. 449, 169-178.

Niemela, J., and K. R. Sreenivasan, 2003a, "Confined turbulent convection," J. Fluid Mech. 481, 355-384.

Niemela, J., and K. R. Sreenivasan, 2006a, "Turbulent convection at high Rayleigh numbers and aspect ratio 4," J. Fluid Mech. 557, 411-422.

Niemela, J., and K. R. Sreenivasan, 2006b, "The use of cryogenic helium for classical turbulence: Promises and hurdles," J. Low Temp. Phys. 143, 163-212.

Niemela, J. J., L. Skrbek, K. R. Sreenivasan, and R. Donnelly, 2000b, "Turbulent convection at very high Rayleigh numbers," Nature (London) 406, 439.

Niemela, J. J., L. Skrbek, C. Swanson, S. Hall, K. R. Sreenivasan, and R. J. Donnelly, 2000, "New results in cryogenic helium flows at ultra-high Reynolds and Rayleigh numbers," J. Low Temp. Phys. 121, 417-422.

Niemela, J. J., and K. R. Sreenivasan, 2003b, "Rayleighnumber evolution of large-scale coherent motion in turbulent convection," Europhys. Lett. 62, 829-833.

Nikolaenko, A., and G. Ahlers, 2003, "Nusselt number measurements for turbulent Rayleigh-Bénard convection," Phys. Rev. Lett. 91, 084501. 
Nikolaenko, A., E. Brown, D. Funfschilling, and G. Ahlers, 2005, "Heat transport by turbulent Rayleigh-Bénard convection in cylindrical cells with aspect ratio one and less," J. Fluid Mech. 523, 251-260.

Oberbeck, A., 1879, "Über die wärmeleitung der flüssigkeiten bei berücksichtigung der strömungen infolge von temperaturdifferenzen," Ann. Phys. Chem. 7, 271.

Obukhov, A. M., 1959, Sov. Phys. Dokl. 4, 61.

Otero, J., R. W. Wittenberg, R. A. Worthing, and C. R. Doering, 2002, "Bounds on Rayleigh-Benard convection with an imposed heat flux," J. Fluid Mech. 473, 191-199.

Perrier, F., P. Morat, and J. L. LeMouel, 2002, "Dynamics of air avalanches in the access pit of an underground quarry," Phys. Rev. Lett. 89, 134501.

Plasting, S. C., and R. R. Kerswell, 2003, "Improved upper bound on the energy dissipation rate in plane Couette flow: The full solution to Busse's problem and the ConstantinDoering-Hopf problem with one-dimensional background field," J. Fluid Mech. 477, 363-379.

Prandtl, L., 1905, "Über Flüssigkeitsbewegungen bei sehr kleiner Reibung," in Verhandlungen des III. Int. Math. Kongr., Heidelberg, 1904 (Teubner, Leipzig), pp. 484-491.

Procaccia, I., E. S. C. Ching, P. Constantin, L. P. Kadanoff, A. Libchaber, and X. Z. Wu, 1991, "Transition to convective turbulence: The role of thermal plumes," Phys. Rev. A 44, 80918102.

Puthenveettil, B. A., G. Ananthakrishna, and J. H. Arakeri, 2005, "The multifractal nature of plume structure in highRayleigh-number convection,” J. Fluid Mech. 526, 245-256.

Puthenveettil, B. A., and J. H. Arakeri, 2005, "Plume structure in high-Rayleigh-number convection," J. Fluid Mech. 542, 217-249.

Puthenveettil, B. A., and J. H. Arakeri, 2008, "Convection due to an unstable density difference across a permeable membrane," J. Fluid Mech. 609, 139-170.

Qiu, X. L., X. D. Shang, P. Tong, and K.-Q. Xia, 2004, "Velocity oscillations in turbulent Rayleigh-Bénard convection,' Phys. Fluids 16, 412-423.

Qiu, X. L., and P. Tong, 2001a, "Large scale velocity structures in turbulent thermal convection," Phys. Rev. E 64, 036304.

Qiu, X. L., and P. Tong, 2001b, "Onset of coherent oscillations in turbulent Rayleigh-Bénard convection," Phys. Rev. Lett. 87, 094501.

Qiu, X. L., and P. Tong, 2002, "Temperature oscillations in turbulent Rayleigh-Bénard convection," Phys. Rev. E 66, 026308.

Qiu, X. L., and K.-Q. Xia, 1998a, "Spatial structure of the viscous boundary layer in turbulent convection," Phys. Rev. E 58, 5816-5820.

Qiu, X. L., and K.-Q. Xia, 1998b, "Viscous boundary layers at the sidewall of a convection cell," Phys. Rev. E 58, 486-491.

Qiu, X. L., K. Q. Xia, and P. Tong, 2005, "Experimental study of velocity boundary layer near a rough conducting surface in turbulent natural convection," J. Turbul. 6, 1-13.

Qiu, X. L., S. H. Yao, and P. Tong, 2000, "Large-scale coherent rotation and oscillation in turbulent thermal convection," Phys. Rev. E 61, R6075-R6078.

Rahmstorf, S., 2000, "The thermohaline ocean circulation: A system with dangerous thresholds?," Clim. Change 46, 247256.

Resagk, C., R. du Puits, A. Thess, F. V. Dolzhansky, S. Grossmann, F. Fontenele Araujo, and D. Lohse, 2006, "Oscillations of the large scale wind in turbulent thermal convection,"
Phys. Fluids 18, 095105.

Roche, P., B. Castaing, B. Chabaud, B. Hebral, and J. Sommeria, 2001, "Side wall effects in Rayleigh-Bénard experiments," Eur. Phys. J. B 24, 405-408.

Roche, P., F. Gauthier, B. Chabaud, and B. Hébral, 2005, "Ultimate regime of convection: Robustness to poor thermal reservoirs," Phys. Fluids 17, 115107.

Roche, P. E., B. Castaing, B. Chabaud, and B. Hebral, 2001, "Observation of the $1 / 2$ power law in Rayleigh-Bénard convection," Phys. Rev. E 63, 045303(R).

Roche, P. E., B. Castaing, B. Chabaud, and B. Hebral, 2002, "Prandtl and Rayleigh numbers dependences in RayleighBénard convection," Europhys. Lett. 58, 693-698.

Roche, P. E., B. Castaing, B. Chabaud, and B. Hebral, 2004, "Heat transfer in turbulent Rayleigh-Bénard convection below the ultimate regime," J. Low Temp. Phys. 134, 1011-1042. Rossby, H. T., 1969, "A study of Bénard convection with and without rotation,” J. Fluid Mech. 36, 309-335.

Sano, M., X. Z. Wu, and A. Libchaber, 1989, "Turbulence in helium-gas gree-convection," Phys. Rev. A 40, 6421-6430.

Schlichting, H., and K. Gersten, 2000, Boundary Layer Theory, 8th ed. (Springer-Verlag, Berlin).

Schmalzl, J., M. Breuer, and U. Hansen, 2002, "The influence of the Prandtl number on the style of vigorous thermal convection," Geophys. Astrophys. Fluid Dyn. 96, 381-403.

Schmalzl, J., M. Breuer, S. Wessling, and U. Hansen, 2004, "On the validity of two-dimensional numerical approaches to time-dependent thermal convection," Europhys. Lett. 67, 390-396.

Shang, X. D., X. L. Qiu, P. Tong, and K.-Q. Xia, 2003, "Measured local heat transport in turbulent Rayleigh-Bénard convection," Phys. Rev. Lett. 90, 074501.

Shang, X.-D., X.-L. Qiu, P. Tong, and K.-Q. Xia, 2004, "Measurements of the local convective heat flux in turbulent Rayleigh-Bénard convection,” Phys. Rev. E 70, 026308.

Shang, X. D., P. Tong, and K.-Q. Xia, 2008, "Scaling of the local convective heat flux in turbulent Rayleigh-Bénard convection," Phys. Rev. Lett. 100, 244503.

Shishkina, O., and C. Wagner, 2006, "Analysis of thermal dissipation rates in turbulent Rayleigh-Bénard convection," J. Fluid Mech. 546, 51-60.

Shishkina, O., and C. Wagner, 2007, "Local heat fluxes in turbulent Rayleigh-Bénard convection,” Phys. Fluids 19, 085107. Shishkina, O., and C. Wagner, 2008, "Analysis of sheetlike thermal plumes in turbulent Rayleigh-Bénard convection," J. Fluid Mech. 599, 383-404.

Shraiman, B. I. and E. D. Siggia, 1990, "Heat transport in highRayleigh number convection," Phys. Rev. A 42, 3650-3653.

Siggia, E. D., 1994, "High Rayleigh number convection," Annu. Rev. Fluid Mech. 26, 137-168.

Sirovich, L., S. Balachandar, and M. R. Maxey, 1989, "Simulation of turbulent thermal convection," Phys. Fluids A 1, 1911-1914.

Spiegel, E. A., 1971, "Convection in stars," Annu. Rev. Astron. Astrophys. 9, 323-352.

Sreenivasan, K. R., A. Bershadski, and J. Niemela, 2002, "Mean wind and its reversals in thermal convection," Phys. Rev. E 65, 056306.

Stevens, R. J. A. M., R. Verzicco, and D. Lohse, 2009, "Radial boundary layer structure and Nusselt number in RayleighBénard convection" (unpublished).

Stringano, G., G. Pascazio, and R. Verzicco, 2006, "Turbulent thermal convection over grooved plates," J. Fluid Mech. 557, 
307-336.

Stringano, G., and R. Verzicco, 2006, "Mean flow structure in thermal convection in a cylindrical cell of aspect-ratio one half," J. Fluid Mech. 548, 1-16.

Sugiyama, K., E. Calzavarini, S. Grossmann, and D. Lohse, 2007, "Non-Oberbeck-Boussinesq effects in Rayleigh-Bénard convection: Beyond boundary-layer theory," Europhys. Lett. 80, 34002 .

Sugiyama, K., E. Calzavarini, S. Grossmann, and D. Lohse, 2009, "Flow organization in non-Oberbeck-Boussinesq Rayleigh-Bénard convection in water," J. Fluid Mech. (in press).

Sun, C., Y. H. Cheung, and K. Q. Xia, 2008, "Experimental studies of the viscous boundary layer properties in turbulent Rayleigh-Bénard convection,” J. Fluid Mech. 605, 79-113.

Sun, C., L.-Y. Ren, H. Song, and K.-Q. Xia, 2005, "Heat transport by turbulent Rayleigh-Bénard convection in $1 \mathrm{~m}$ diameter cylindrical cells of widely varying aspect ratio," J. Fluid Mech. 542, 165-174.

Sun, C., H. D. Xi, and K. Q. Xia, 2005, “Azimuthal symmetry, flow dynamics, and heat transport in turbulent thermal convection in a cylinder with an aspect ratio of 0.5 ," Phys. Rev. Lett. 95, 074502.

Sun, C., and K.-Q. Xia, 2005, "Scaling of the Reynolds number in turbulent thermal convection," Phys. Rev. E 72, 067302.

Sun, C., K. Q. Xia, and P. Tong, 2005, "Three-dimensional flow structures and dynamics of turbulent thermal convection in a cylindrical cell," Phys. Rev. E 72, 026302.

Sun, C., Q. Zhou, and K. Q. Xia, 2006, "Cascades of velocity and temperature fluctuations in buoyancy-driven thermal turbulence," Phys. Rev. Lett. 97, 144504.

Takeshita, T., T. Segawa, J. A. Glazier, and M. Sano, 1996, "Thermal turbulence in mercury," Phys. Rev. Lett. 76, 14651468 .

Theerthan, S. A., and J. H. Arakeri, 1998, “A model for nearwall dynamics in turbulent Rayleigh-Bénard convection," J. Fluid Mech. 372, 221-254.

Theerthan, S. A., and J. H. Arakeri, 2000, "Planform structure and heat transfer in turbulent free convection over horizontal surfaces," Phys. Fluids 12, 884-894.

Threlfall, D. C., 1975, "Free convection in low temperature gaseous helium," J. Fluid Mech. 67, 17-28.

Tilgner, A., A. Belmonte, and A. Libchaber, 1993, "Temperature and velocity profiles of turbulence convection in water," Phys. Rev. E 47, R2253-R2256.

Tsai, P., Z. A. Daya, V. B. Deyirmenjian, and S. W. Morris, 2007, "Direct numerical simulation of supercritical annular electroconvection," Phys. Rev. E 76, 026305.

Tsai, P., Z. A. Daya, and S. W. Morris, 2003, “Aspect-ratio dependence of charge transport in turbulent electroconvection," Phys. Rev. Lett. 92, 084503.

Tsai, P., Z. A. Daya, and S. W. Morris, 2005, "Charge transport scaling in turbulent electroconvection," Phys. Rev. E 72, 046311.

van Doorn, E., B. Dhruva, K. R. Sreenivasan, and V. Cassella, 2000, "Statistics of wind direction and its increments," Phys. Fluids 12, 1529.

Verzicco, R., 2002, "Sidewall finite conductivity effects in confined turbulent thermal convection," J. Fluid Mech. 473, 201210.

Verzicco, R., 2003, "Turbulent thermal convection in a closed domain: Viscous boundary layer and mean flow effects," Eur. Phys. J. B 35, 133-141.
Verzicco, R., 2004, "Effect of non-perfect thermal sources in turbulent thermal convection," Phys. Fluids 16, 1965-1979.

Verzicco, R., and R. Camussi, 1997, "Transitional regimes of low-Prandtl thermal convection in a cylindrical cell," Phys. Fluids 9, 1287-1295.

Verzicco, R., and R. Camussi, 1999, "Prandtl number effects in convective turbulence," J. Fluid Mech. 383, 55-73.

Verzicco, R., and R. Camussi, 2003, "Numerical experiments on strongly turbulent thermal convection in a slender cylindrical cell," J. Fluid Mech. 477, 19-49.

Verzicco, R., and K. R. Sreenivasan, 2008, “A comparison of turbulent thermal convection between conditions of constant temperature and constant heat flux," J. Fluid Mech. 595, 203219.

Villermaux, E., 1995, "Memory-induced low frequency oscillations in closed convection boxes," Phys. Rev. Lett. 75, 46184621.

Wang, J., and K.-Q. Xia, 2003, "Spatial variations of the mean and statistical quantities in the thermal boundary layers of turbulent convection," Eur. Phys. J. B 32, 127-136.

Werne, J., 1993, "Structure of hard-turbulent convection in two-dimensions: Numerical evidence," Phys. Rev. E 48, 10201035.

Werne, J., E. E. DeLuca, R. Rosner, and F. Cattaneo, 1991, "Development of hard-turbulence convection in two dimensions-Numerical evidence," Phys. Rev. Lett. 67, 35193522.

Wu, X. Z., L. Kadanoff, A. Libchaber, and M. Sano, 1990, "Frequency power spectrum of temperature-fluctuation in free-convection," Phys. Rev. Lett. 64, 2140-2143.

Wu, X. Z., and A. Libchaber, 1991, "Non-Boussinesq effects in free thermal convection," Phys. Rev. A 43, 2833-2839.

Wu, X. Z., and A. Libchaber, 1992, "Scaling relations in thermal turbulence: The aspect ratio dependence," Phys. Rev. A 45, 842-845.

Xi, H. D., S. Lam, and K.-Q. Xia, 2004, "From laminar plumes to organized flows: The onset of large-scale circulation in turbulent thermal convection," J. Fluid Mech. 503, 47-56.

Xi, H. D., and K. Q. Xia, 2007, "Cessations and reversals of the large-scale circulation in turbulent thermal convection," Phys. Rev. E 75, 066307.

Xi, H. D., and K. Q. Xia, 2008a, "Azimuthal motion, reorientation, cessation, and reversal of the large-scale circulation in turbulent thermal convection: A comparison between aspect ratio one and one-half geometries," Phys. Rev. E 78, 036326. Xi, H. D., and K. Q. Xia, 2008b, "Flow mode transitions in turbulent thermal convection," Phys. Fluids 20, 055104.

Xi, H.-D., Q. Zhou, and K.-Q. Xia, 2006, “Azimuthal motion of the mean wind in turbulent thermal convection," Phys. Rev. E 73, 056312.

Xi, H. D., S. Q. Zhou, Q. Zhou, T. S. Chan, and K. Q. Xia, 2009, "Origin of the temperature oscillations in turbulent thermal convection," Phys. Rev. Lett. 102, 044503.

Xia, K.-Q., S. Lam, and S. Q. Zhou, 2002, "Heat-flux measurement in high-Prandtl-number turbulent Rayleigh-Bénard convection," Phys. Rev. Lett. 88, 064501.

Xia, K.-Q., and S.-L. Lui, 1997, "Turbulent thermal convection with an obstructed sidewall," Phys. Rev. Lett. 79, 5006-5009. Xia, K.-Q., and X. L. Qiu, 1999, "Turbulent convection with "disconnected" top and bottom boundary layers," Europhys. Lett. 46, 171-176.

Xia, K.-Q., C. Sun, and S. Q. Zhou, 2003, "Particle image velocimetry measurement of the velocity field in turbulent ther- 
mal convection,” Phys. Rev. E 68, 066303.

Xin, Y. B., and K.-Q. Xia, 1997, "Boundary layer length scales in convective turbulence," Phys. Rev. E 56, 3010-3015.

Xin, Y. B., K.-Q. Xia, and P. Tong, 1996, "Measured velocity boundary layers in turbulent convection," Phys. Rev. Lett. 77, 1266-1269.

Xu, X., K. M. S. Bajaj, and G. Ahlers, 2000, "Heat transport in turbulent Rayleigh-Bénard convection,” Phys. Rev. Lett. 84, 4357-4360.

Yakhot, V., 1992, “4/5 Kolmogorov law for statistically stationary turbulence: Application to high Rayleigh number Bénard convection," Phys. Rev. Lett. 69, 769-771.

Yu, H., N. Li, and R. E. Ecke, 2007, "Scaling in laminar natural convection in laterally heated cavities: Is turbulence essential in the classical scaling of heat transfer?," Phys. Rev. E 76, 026303.

Zaleski, S., 1998, in Geophysical and Astrophysical Convection, edited by P. Fox, and R. Kerr (Gordon and Breach Science, New York), pp. 129-143.

Zhang, J., S. Childress, and A. Libchaber, 1997, "NonBoussinesq effect: Thermal convection with broken symmetry," Phys. Fluids 9, 1034-1042.

Zhong, J. Q., R. J. A. M. Stevens, H. J. H. Clercx, R. Verzicco, D. Lohse, and G. Ahlers, 2009, "Prandtl-, Rayleigh-, and Rossby-number dependence of heat transport in turbulent rotating Rayleigh-Bénard convection," Phys. Rev. Lett. 102, 044502.

Zhou, S.-Q., C. Sun, and K.-Q. Xia, 2007a, "Measured oscillations of the velocity and temperature fields in turbulent Rayleigh-Bénard convection in a rectangular cell," Phys. Rev. E 76, 036301

Zhou, Q., C. Sun, and K.-Q. Xia, 2007b, "Morphological evolution of thermal plumes in turbulent Rayleigh-Bénard convection," Phys. Rev. Lett. 98, 074501.

Zhou, Q., C. Sun, and K. Q. Xia, 2008, "Experimental investigations of homogeneity, isotropy, and circulation of the velocity field in buoyancy-driven turbulence," J. Fluid Mech. 598, 361-372.

Zhou, Q., H. D. Xi, S. Q. Zhou, C. Sun, and K. Q. Xia, 2009, "Oscillations of the large-scale circulations in turbulent Rayleigh-Bénard convection: The sloshing mode and its relationship with the torsional mode," J. Fluid Mech. (to be published).

Zhou, S. Q., and K.-Q. Xia, 2002, "Plume statistics in thermal turbulence: Mixing of an active scalar," Phys. Rev. Lett. 89, 184502.

Zocchi, G., E. Moses, and A. Libchaber, 1990, "Coherent structures in turbulent convection: An experimental study," Physica A 166, 387-407. 